\title{
Frequency and Criticality of Diagnoses in Family Medicine Practices: From the National Ambulatory Medical Care Survey (NAMCS)
}

\author{
Michael R. Peabody, PhD, Thomas R. O'Neill, PhD, Keith L. Stelter, MD, MMM, \\ and James C. Puffer, $M D$
}

Background: Family medicine is a specialty of breadth, providing comprehensive health care for the individual and the family that integrates the broad scope of clinical, social, and behavioral sciences. As such, the scope of practice (SOP) for family medicine is extensive; however, over time many family physicians narrow their SOP. We sought to provide a nationally representative description of the most common and the most critical diagnoses that family physicians see in their practice.

Methods: Data were extracted from the 2012 National Ambulatory Medical Care Survey (NAMCS) to select all ICD-9 codes reported by family physicians. A panel of family physicians then reviewed 1893 ICD-9 codes to place each code into an American Board of Family Medicine Family Medicine Certification Examination test plan specifications (TPS) category and provide a rating for an Index of Harm (IoH).

Results: An analysis of all 1893 ICD-9 codes seen by family physicians in the 2012 NAMCS found that 198 ICD-9 codes could not be assigned a TPS category, leaving 1695 ICD-9 codes in the dataset. Top 10 lists of ICD-9 codes by TPS category were created for both frequency and IoH.

Conclusions: This study provides a nationally representative description of the most common diagnoses that family physicians are seeing in their practice and the criticality of these diagnoses. These results provide insight into the domain of the specialty of family medicine. Medical educators may use these results to better tailor education and training to practice. (J Am Board Fam Med 2018;31: 126-138.)

Keywords: Ambulatory Care, Behavioral Sciences, Certification, Comprehensive Health Care, Family Physicians, Health Care Surveys, International Classification of Diseases

Family medicine is a specialty of breadth, providing comprehensive health care for both the individual and the family that integrates the broad scope of clinical, social, and behavioral sciences. ${ }^{1}$ As such, the scope of practice (SOP) for family medicine is extensive. Generally speaking, the scope of the specialty has been characterized by the Accreditation Council for Graduate Medical Education training

This article was externally peer reviewed.

Submitted 24 May 2017; revised 16 August 2017; accepted 24 August 2017.

From the American Board of Family Medicine, Lexington, KY (MRP, TRO, JCP); University of Minnesota Mankato Family Medicine Residency Program, Mankato, MN (KLS).

Funding: none.

Conflict of interest: none declared.

Corresponding author: Michael R. Peabody, $\mathrm{PhD}$, American Board of Family Medicine, 1648 McGrathiana Pkwy, ste 550, Lexington, KY (E-mail: mpeabody@theabfm.org). requirements ${ }^{2}$ and the American Board of Family Medicine (ABFM) certification examination blueprint. $^{3}$

Although family physicians are trained to address a wide variety of medical problems, over time many family physicians narrow their SOP. Currently, fewer family physicians are providing obstetric care ${ }^{4}$, women's health ${ }^{5}$, and pediatric care ${ }^{6}$ than they have in the past. Narrowing of a physician's SOP can occur for a variety of reasons, such as needs of the community, physician preference, or employer requirements.

In some cases, a physician may have narrowed his or her SOP while still believing she or he predominantly practices full-scope family medicine. Cognitive psychology describes a phenomenon called the Availability Heuristic ${ }^{7}$, which causes 
people to make judgments about the likelihood of an event based on how easily an example comes to mind. Being able to easily recall a few patients who seemingly represent full-spectrum care would make a physician believe she or he is practicing fullspectrum care when, in fact, she or he is not. The availability of information in memory also underlies the Representativeness Heuristic. ${ }^{8}$ With the Representativeness Heuristic, people judge the probability of an event belonging to a certain class based on the degree to which the event resembles the class; however, this neglects the probability of the class occurring in the first place. Physicians also change practice patterns and scope gradually so changes in their SOP are not noticeable, unless they have a method to benchmark their practice against a standard. It would be helpful for these physicians to have information about the domain of family medicine that they could compare with their own practice to get a more accurate picture of their individual SOP.

Furthermore, it would be helpful for medical educators in family medicine to have accurate information about the domain of family medicine to more fully target their training to reflect the conditions that their residents will be expected to treat once they are no longer under the residency program's supervision. Finally, in constructing an examination designed to certify family physicians, it is critical that the construct adequately, accurately, and fairly assess the domain of family medicine.

The National Center for Health Statistics conducts the National Ambulatory Medical Care Survey (NAMCS) annually. ${ }^{9}$ NAMCS is a large, representative, national sample survey that provides information about ambulatory care delivered in the United States. This publicly available dataset includes the physician reported International Classification of Diseases, Ninth Revision, Clinical Modification (ICD-9 $)^{10}$ codes for each patient visit in the sample and permits stratification by physician specialty. This dataset is well suited to describing the patient visits commonly seen by family physicians.

Using the NAMCS dataset, this article will provide a nationally representative description of the types of diagnoses that were seen by family physicians in ambulatory care settings in 2012 and estimates of the prevalence of those diagnoses. To supplement this perspective, an indicator of the degree of harm that would be caused by misdiag- nosing or incorrectly treating each diagnosis was derived from ratings made by a panel of family physicians. Because of the large number of ICD-9 codes available, it may be of some utility to aggregate them into clinically relevant categories. We chose to use the ABFM's Family Medicine Certification Examination (FMCE) test plan specifications (TPS) because approximately $85 \%$ of active family physicians are certified by the $\mathrm{ABFM}^{11}$, making it quite possibly the most widely applicable framework available.

\section{Methods \\ Design}

This study used the 2012 NAMCS data to identify ICD-9 codes that were reported by family physicians. These ICD-9 codes were included on a survey that was administered to physicians so that the ICD-9 codes could be linked to the ABFM's FMCE TPS categories. Each ICD-9 code was either linked to a TPS category or excluded for being too vague. NAMCS provides a weight for each visit in the sample (called a patient weight), allowing for the calculation of the frequency with which each ICD-9 was reported in the population. The top 10 most frequently seen ICD-9 codes for each TPS category are reported. In addition, the survey also asked the physicians about the likely degree of harm that would be caused by misdiagnosing or incorrectly treating the ICD-9 code. This information was transformed into an Index of Harm (IoH) scale.

\section{NAMCS Data}

The sampling frame for the 2012 NAMCS was composed of all physicians contained in the American Medical Association and the American Osteopathic Association master files who were office based; principally engaged in patient care activities; nonfederally employed; not in the specialties of anesthesiology, pathology, and radiology; and younger than 85 years of age at the time of the survey.

The 2012 NAMCS sample included 15,740 physicians: 14,931 allopathic physicians and 809 osteopathic physicians. Physicians included in the sample were screened at the time of the survey to assure that they met the above-mentioned criteria. A total of 6166 physicians did not meet all the criteria and were ruled ineligible for the study. Of the 9,574 
eligible physicians, 3,583 participated in the NAMCS survey. For these, data were collected for 76,330 visits, either by a NAMCS representative $(74,647$ visits) or by physicians or their staffs $(1,683$ visits). ${ }^{12}$

We restricted our sample to those physicians whose self-identified specialty was family practice or general practice, as defined by NAMCS. Of the 76,330 Patient Record Forms (PRF) and 3,583 physicians, there remained 12,897 PRFs provided by 551 physicians following the implementation of the inclusion criterion. NAMCS contains a patient weight estimate that is used to obtain visit estimates from the survey data. By aggregating the patient weights, we are able to obtain estimates for the approximately $928,629,953$ office visits made in the United States in 2012. Subsequent analyses were based on this dataset.

\section{Panelists}

An invitation was sent to $100 \mathrm{ABFM}$-certified family physicians from the greater Minneapolis, MN area to participate in a 1-day panel to review a sample 800 ICD-9 codes from a relevant population of 1893 ICD-9 codes. There were 27 physicians who accepted the invitation and 21 participated. The exercise lasted a single day and the panelists were provided an honorarium to acknowledge their contribution.

\section{Instrumentation}

Survey Content

A master survey was created using the ICD-9 codes that were reported by the physicians in the NAMCS sample for each patient visit; in aggregate, 1893 unique ICD-9 codes were reported. Given that the purpose of the survey was to connect the ICD-9 codes with the ABFM's FMCE TPS categories as a framework for understanding the domain of family medicine, panelists were asked to assign each ICD-9 code to 1 of the 16 FMCE TPS categories: Respiratory, Cardiovascular, Musculoskeletal, Gastrointestinal, Special Sensory (visual, hearing, etc.), Endocrine, Integumentary, Neurologic (brain, spinal cord, peripheral nervous system), Psychogenic (psychological, behavioral, mental health), Reproductive (male), Reproductive (female), Nephrologic, Hematologic/Immune, Nonspecific, Population-Based Care and Health Systems, and Patient-Based Care and Systems.
In addition to providing a TPS category for each ICD-9 code, panelists were also asked to answer, "How critical is the diagnosis and treatment of this condition?" using a 4-point Likert-type rating scale (Minimally, Moderately, Somewhat, Very). These data were used to create an Index of Harm (IoH) scale.

\section{Survey Data Collection Design}

Because asking panelists to review all 1893 ICD-9 codes from the master survey would be burdensome and time consuming, each panelist was asked to review only a subset of the codes. To accomplish this, the 1893 ICD-9 codes were grouped into blocks of 100 and staggered to implement a sampling strategy that assigned these blocks of ICD-9 codes to panelists such that each code was rated by as few as 6 or as many as 12 panelists (Figure 1). Each panelist received 400 codes in the morning and 400 in the afternoon. The survey was also designed to provide overlap of ICD-9 codes across raters to ensure that adjustments of rater severity applied to the $\mathrm{IoH}$ would be from the same frame of reference (Figure 1). ${ }^{13,14}$ Furthermore, the IoH was estimated using the Rasch measurement ${ }^{15}$ Rating Scale Model ${ }^{16,17}$, which we used to adjust for the severity of the individual raters. It is important to note that Rasch models are robust to missing data, so the incomplete data design is not problematic when the overlap is sufficient to establish connectivity.

\section{Analysis}

\section{Panel Review}

To determine the category into which an ICD-9 code should be classified, the panelists' individual classifications were aggregated. For each ICD-9 code, if the total percentage of ratings for any single category was greater than or equal to $70 \%$ then that ICD-9 code was determined to have a sufficient level of agreement among the panelists to be classified in that category. The $70 \%$ consensus threshold was selected because the threshold had to be greater than $50 \%$ to establish that it was the dominant choice, but expecting $100 \%$ consensus in all cases seemed unreasonable. Furthermore, some codes were rated by as few as 6 or 7 panelists. Under these conditions the granularity of percentage in agreement becomes very coarse. With 6 raters, the possible choices are: $67 \%$ or $83 \%$ agreement. With 7 raters, the possible choices are: $57 \%$, 
Figure 1. Data collection matrix by rater ID number, 100 item International Classification of Diseases, Ninth Revision (ICD-9) code block, and morning/afternoon schedule designed to ensure overlap of ratings for connectivity.

\begin{tabular}{|c|c|c|c|c|c|c|c|c|c|c|c|c|c|c|c|c|c|c|c|c|c|c|c|c|c|}
\hline & 1 & 2 & 3 & 4 & 5 & 6 & 7 & 8 & 9 & 10 & 12 & 14 & 15 & \begin{tabular}{|l|l}
16 \\
\end{tabular} & 18 & 19 & 20 & 21 & 22 & 23 & 24 & $26 ?$ & AM $P$ & $M$ & TOTAI \\
\hline $1-100$ & $\mathrm{AM}$ & & & & & & & & & & & PM & EM & PM 2 & AM & AM & $\mathrm{AM}$ & & & PM & & PM & 4 & 5 & \\
\hline $101-200$ & AM & AM & & & & & & & & & & PM & PM & PM 2 & $A M$ & AM & AM & & & PM & & $\mathrm{PM}$ & 5 & 5 & \\
\hline $201-300$ & AM & AM & AM & & & & & & & & & & EM & PM & PM & AM & AM & & & PM & & DM & 5 & 5 & \\
\hline $301-400$ & $\mathrm{AM}$ & AM & AM & AM & & & & & & & & & & \begin{tabular}{|l|l} 
PM & . \\
\end{tabular} & PM & PM & $A M$ & & & PM & & $\mathrm{PM}$ & 5 & 5 & \\
\hline $401-500$ & PM & AM & AM & AM & AM & & & & & & & & & & PM & PM & PM & AM & & & $\mathrm{PM}$ & $A M$ & 6 & 5 & \\
\hline $501-600$ & PM & PM & AM & AM & AM & $\mathrm{AM}$ & & & & & & & & & PM & PM & \begin{tabular}{|l|l|l} 
PM & \\
\end{tabular} & $\mathrm{AM}$ & & & PM & $A M$ & 6 & 6 & \\
\hline $601-700$ & PM & PM & PM & $A M$ & $A M$ & AM & $A M$ & & & & & & & & & PM & PM & $A M$ & & & PM & $A M$ & 6 & 6 & \\
\hline $701-800$ & PM & PM & PM & PM & AM & AM & \begin{tabular}{|l|l} 
AM & \\
\end{tabular} & $\mathrm{AM}$ & & & & & & & & & PM & AM & & & PM & $\mathrm{AM}$ & 6 & 6 & \\
\hline $801-900$ & & PM & $\mathrm{PM}$ & PM & PM & AM & AM & $\mathrm{AM}$ & $A M$ & & & & & & & & & PM & AM & & & & 5 & 5 & \\
\hline $901-1000$ & & & $\mathrm{PM}$ & PM & PM & PM & AM & AM & $A M$ & AM & & & & & & & & PM 2 & AM & & & & 5 & - & \\
\hline $1001-1100$ & & & & PM & PM & PM & PM & AM & $A M$ & AM & & & & & & & & PM & AM & & & & 4 & 5 & \\
\hline $1101-1200$ & & & & & PM & PM & PM & PM & AM & AM & $A M$ & & & & & & & PM & AM & & & & 4 & 5 & \\
\hline $1201-1300$ & & & & & & PM & PM & PM & PM & $\mathrm{AM}$ & $\mathrm{AM}$ & & & & & & & & PM & AM & & & 3 & 5 & \\
\hline $1301-1400$ & & & & & & & PM & PM & PM & PM & $\mathrm{AM}$ & $\mathrm{AM}$ & & & & & & & PM & $\mathrm{AM}$ & & & 3 & 5 & \\
\hline $1401-1500$ & & & & & & & & PM & PM & PM & $\mathrm{AM}$ & AM & AM & & & & & & PM & AM & & & 4 & 4 & \\
\hline $1501-1600$ & & & & & & & & & PM & PM & $\mathrm{PM}$ & $\mathrm{AM}$ & AM & $\mathrm{AM}$ & & & & & PM & $\mathrm{AM}$ & $\mathrm{AM}$ & & 5 & 4 & \\
\hline $1601-1700$ & & & & & & & & & & PM & PM & $\mathrm{AM}$ & $A M$ & $\mathrm{AM}$ & & & & & & & $\mathrm{AM}$ & & 4 & 2 & \\
\hline $1701-1800$ & & & & & & & & & & & $\mathrm{PM}$ & PM & AM & AM & $A M$ & & & & & & $A M$ & & 4 & 2 & \\
\hline $1801-1900$ & & & & & & & & & & & PM & PM & PM & \begin{tabular}{|l|l}
$\mathrm{M}$ & 2 \\
\end{tabular} & AM & AM & & & & & AM & & 4 & 3 & \\
\hline AM & $z$ & 4 & 4 & 4 & 4 & 4 & 4 & $z$ & 4 & 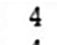 & 4 & 4 & 4 & 4 & 4 & 4 & 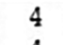 & $\therefore$ & 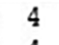 & 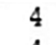 & 4 & 4 & & & \\
\hline PM & 4 & 4 & 4 & 4 & 4 & 4 & 4 & 4 & 4 & $A$ & 4 & 4 & 4 & 4 & 4 & 4 & 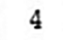 & 4 & 4 & 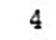 & 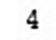 & & & & \\
\hline TOTAL & 8 & 8 & 8 & 8 & 8 & 8 & 8 & 8 & 0 & 8 & 8 & 8 & & & 8 & 8 & 8 & 8 & & 8 & 8 & 8 & & & \\
\hline
\end{tabular}

$71 \%$, or $86 \%$ agreement. We chose $70 \%$ agreement as a compromise between being theoretically acceptable and technically manageable.

\section{Committee Review}

Those ICD-9 codes that did not achieve TPS category classification consensus among the panelists were reviewed by the ABFM Examination Committee using the same instrument and method; however, an additional category of "Too Vague to be Useful" was added. The same $70 \%$ consensus threshold was applied and any ICD-9 code that did not achieve a consensus rating in this review was removed from the final analysis.

\section{Frequencies}

After receiving a final category classification following the ABFM Examination Committee review, the prevalence of each ICD-9 code was examined by conducting frequency counts using the patient weights to estimate the number of visits nationally that were attributable to each ICD-9 code. Because NAMCS allows up to 3 ICD-9 codes to be entered for any single visit, we counted each ICD-9 code occurrence as a separate instance rather than try to control for co- and multi-morbidities.
$\mathrm{IoH}$

The IoH for each ICD-9 code was estimated using a Rasch Rating Scale Model. More specifically, the probability of panelist $n$ with a severity of $\beta$ placing ICD-9 code $i$ with a degree of harm $\delta$ into category $x$ as:

$$
\operatorname{Pr}\left\{X_{n i}=x\right\}=\frac{\exp \sum_{k=0}^{x}\left(\beta_{n}-\left(\delta_{i}+\tau_{k}\right)\right)}{\sum_{j=0}^{m} \exp \sum_{k=0}^{x}\left(\beta_{n}-\left(\delta_{i}+\tau_{k}\right)\right)},
$$

where the categories are ordered from 0 to $m$, and $\tau_{k}$ are the rating scale structure parameters ("Rasch thresholds"). In the Rasch Rating Scale Model, the same rating scale structure is imposed across all ICD-9 codes. Winsteps Rasch Measurement Calibration software version 3.92.1 (Beaverton, OR) was used to calibrate these parameters using the panelists' data.

Because the Rasch model uses logits as its unit of measure, the logit calibrations were linearly transformed to have a minimum value of 0 (actually 0.1 , near 0 ) for the lowest $\mathrm{IoH}$ score and 100 for the highest IoH score to improve the understandability of the measure using the formula: 


$$
\mathrm{IoH}=\left(7 * \delta_{i}\right)+46 .
$$

We examined data-model fit using outlier-sensitive standardized fit statistics (OUTFIT ZSTD), which are normally distributed z-scores showing the improbability of the data if the data were to fit the model perfectly. ${ }^{18}$ OUTFIT ZSTD values above 2.0 are typically considered to be too unpredictable and values below -2.0 are generally considered to be too constrained. ${ }^{19}$ In addition, summary statistics for a person reliability and item reliability are provided. ${ }^{20,21}$

\section{Results \\ Panel Review}

Of the 1893 ICD-9 codes, 1446 (76.4\%) had a single category for which the number ratings met or exceeded $70 \%$ of the total ratings, and were subsequently classified as having reached consensus regarding the appropriate classification category. Each of these ICD-9 codes received at least 4 ratings and as many as 13 ratings. Although panelists were instructed to assign each ICD-9 code to only 1 category, there were 26 instances in which a panelist provided multiple categories; these were allowed to stand and the consensus percentage was calculated by the number of ratings not the number of panelists.

\section{Committee Review}

The ABFM Examination Committee reviewed the 447 ICD-9 codes that did not achieve a consensus rating. The ABFM Examination Committee was not able to reach the $70 \%$ consensus level for 85 codes, which were removed from the final dataset. The ABFM Examination Committee also determined that an additional 113 ICD-9 codes did not provide any meaningful information, which were also removed from the final dataset. Following this review, 1695 ICD-9 codes were assigned a final category classification and can be found in online Appendix A. A list of the 198 ICD-9 codes removed from the final dataset can be found in online Appendix B.

\section{Frequencies}

Estimates of the number of annual visits for each ICD-9 code were calculated using the patient weights in the NAMCS dataset. The 10 most frequently occurring ICD-9 codes assigned to each
TPS category are provided in Table 1. Overall, Unspecified essential hypertension (4019), Other and unspecified hyperlipidemia (2724), and Diabetes mellitus w/o complication (25000) were the most frequently seen diagnoses. The distribution of diagnosis frequency can be seen in Figure 2, which shows that there are approximately 100 diagnoses that family physicians see often and then a lot of infrequently seen diagnoses.

\section{IoH}

The ICD-9 codes with the 10 highest (i.e., most critical) IoH scores assigned to each TPS category are also provided in Table 2. Overall, Systolic heart failure (42821), Diabetes with other coma (25032), and Unspecified intracranial hemorrhage (4329) were found to have the highest $\mathrm{IoH}$ scores. The mean IoH score was 46.6 with a standard deviation of 14.5, which approximates a normal distribution about the mean. The mean OUTFIT ZSTD was -0.2 with 173 ICD-9 codes $(9.1 \%)$ showing an OUTFIT ZSTD value above 2.0 and 103 (5.4\%) below -2.0 . Person reliability was 0.99 and item reliability was 0.86 . There were 15 instances in which a panelist provided multiple $\mathrm{IoH}$ category ratings for a single ICD-9 code; these were classified as missing.

\section{Discussion}

Using a national probability survey of patient visits for office-based family physicians and a panel of family physicians to rate ICD-9 codes for IoH, we were able to examine both the frequency with which diagnoses were seen by family physicians and identify those diagnoses that occur less frequently but have high IoH scores in clinical practice.

The NAMCS data allows us to more accurately describe the breadth of family medicine. As seen in Figure 2, there are approximately 100 diagnoses that family physicians see frequently in office visits and an additional 1600 diagnoses that are seen less often but still occur in office-based visits. Although some diagnoses are seen rarely, they are still important to the SOP of family physicians. The IoH attempts to describe the degree of harm that would be caused by misdiagnosing or incorrectly treating a specific ICD-9 code. For example, "Acute myocardial infarction of other anterior wall, initial episode of care (41011)" received an $\mathrm{IoH}$ score of 93, which was the 10th highest of all ICD-9 codes; 


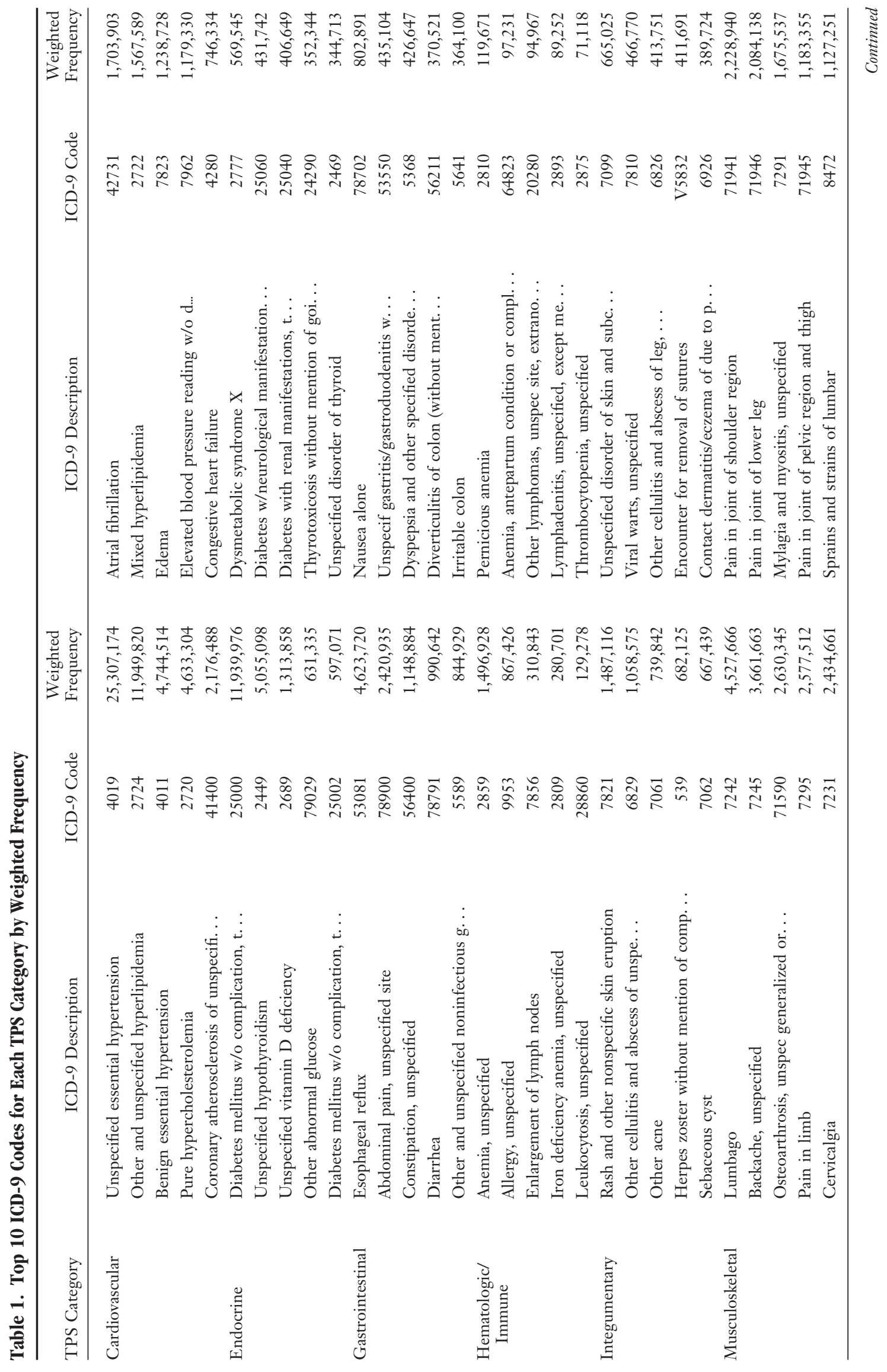

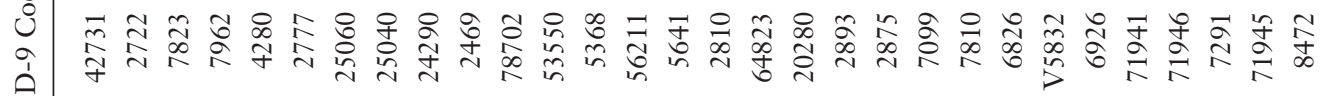




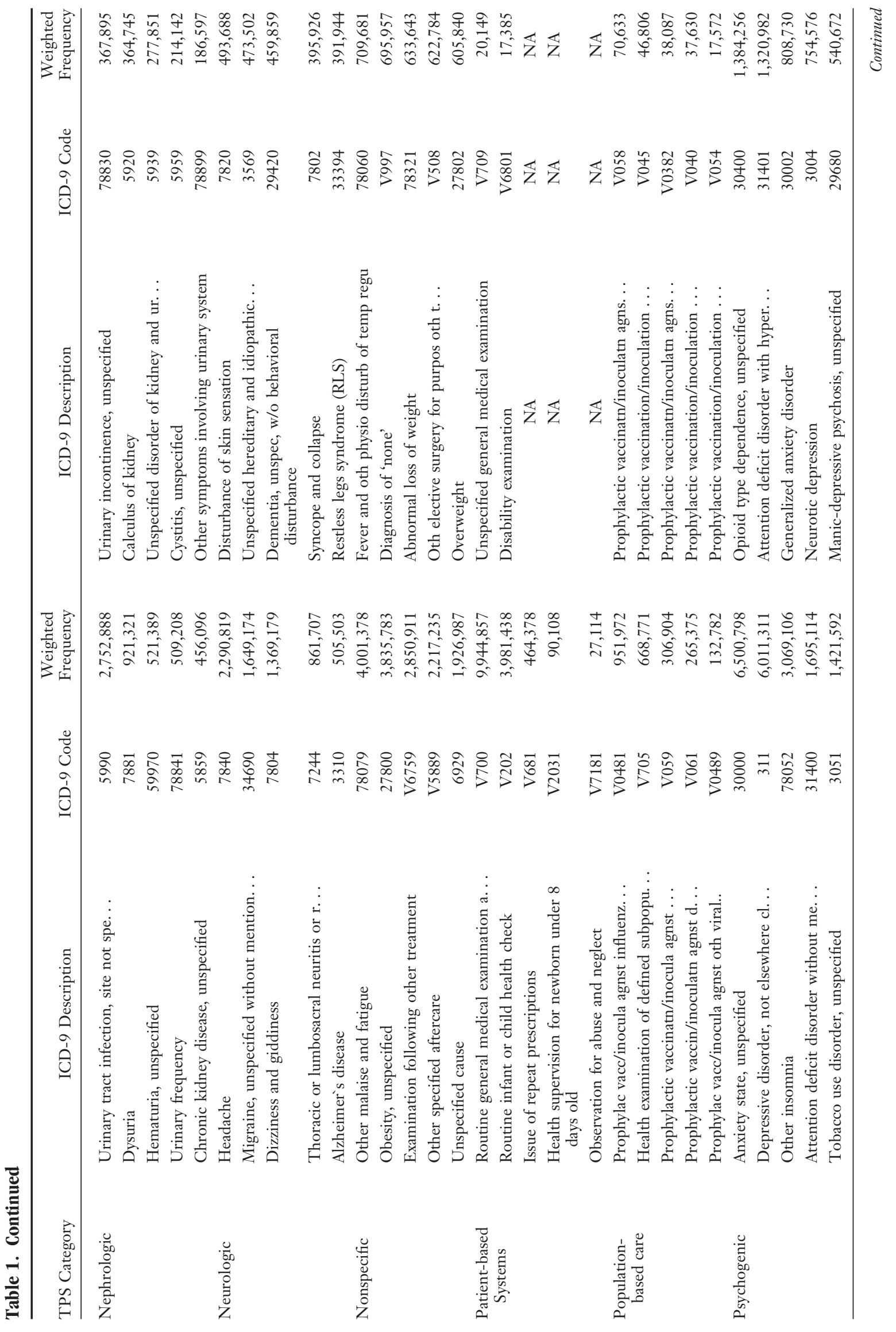




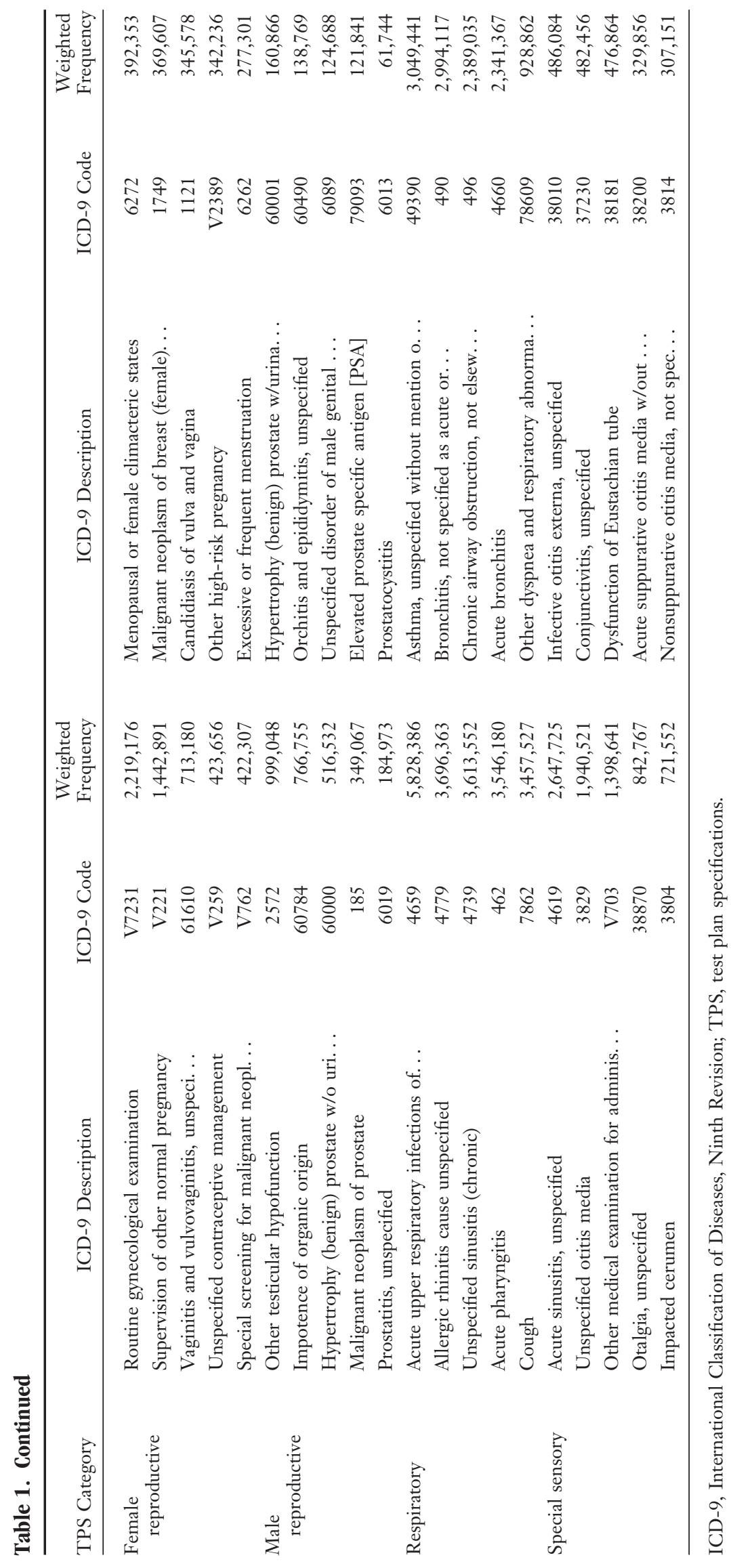


Figure 2. Distribution of International Classification of Diseases, Ninth Revision (ICD-9) codes by frequency.

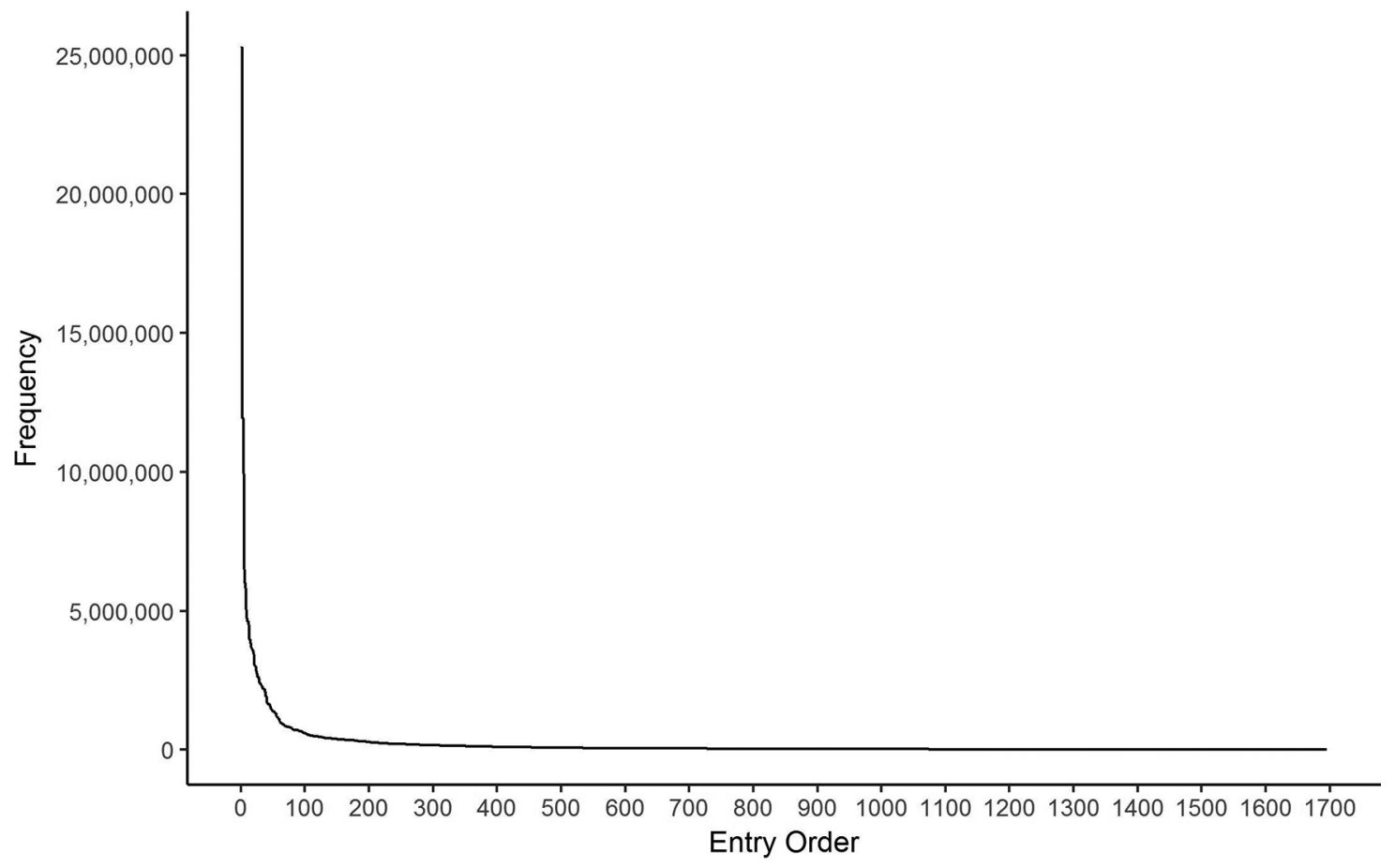

however, it had the second lowest frequency of any diagnosis. This would seem to represent a diagnosis that although rarely seen is an important aspect of the scope of the domain of family medicine.

Individual physicians should be able to use tables 1 and 2 to examine whether their SOP mirrors that of the domain of family medicine while blunting the effects of the Representativeness Heuristic. However, they will still need to accommodate the Availability Heuristic by performing a record review or some other kind of objective analysis rather than relying on their own memory.

Because of the large number of ICD-9 codes, it made sense to aggregate the codes into a common framework using the ABFM FMCE TPS categories. Table 1 provides the top 10 ICD-9 codes by frequency and table 2 provides the top 10 ICD-9 codes by IoH. By using the TPS categories, medical educators may find common themes that would aid in curriculum development and targeting training to practice. For instance, it is likely that during training a resident would encounter the most frequently seen ICD-9 codes in the Cardiology TPS category, such as hypertension, hyperlipidemia, edema, and atrial fibrillation (see Table 1). However, residents may be less likely to encounter some of the conditions with high $\mathrm{IoH}$ scores that occur less frequently, such as acute myocardial infarction, dissection of coronary artery, or acute systolic heart failure (see Table 2). Designing educational programs around these low-frequency, high-IoH conditions may help better prepare residents for these rare, but critical events.

This study has several limitations. The list of ICD-9 codes taken from the NAMCS dataset is clearly not exhaustive, but it is representative and can provide useful information for examining the domain of family medicine. NAMCS considers only family physicians in the outpatient setting and future studies may wish to include the National Hospital Ambulatory Medical Care Survey to better understand the role of family physicians in the hospital/inpatient setting. Furthermore, the single panel site and $21 \%$ participation rate may have introduced some selection bias; however, because ICD-9 codes are used nationally, we have no reason to believe that physicians use them differently. We can think of no reason why this would affect the results. Finally, the ABFM TPS framework, although perhaps not optimal, does seem to represent the best option for a shared framework. Other classification schemes, such as the Agency for Health care Research and Quality's Clinical Classifications Software, may also provide useful representations of the domain of family medicine. 


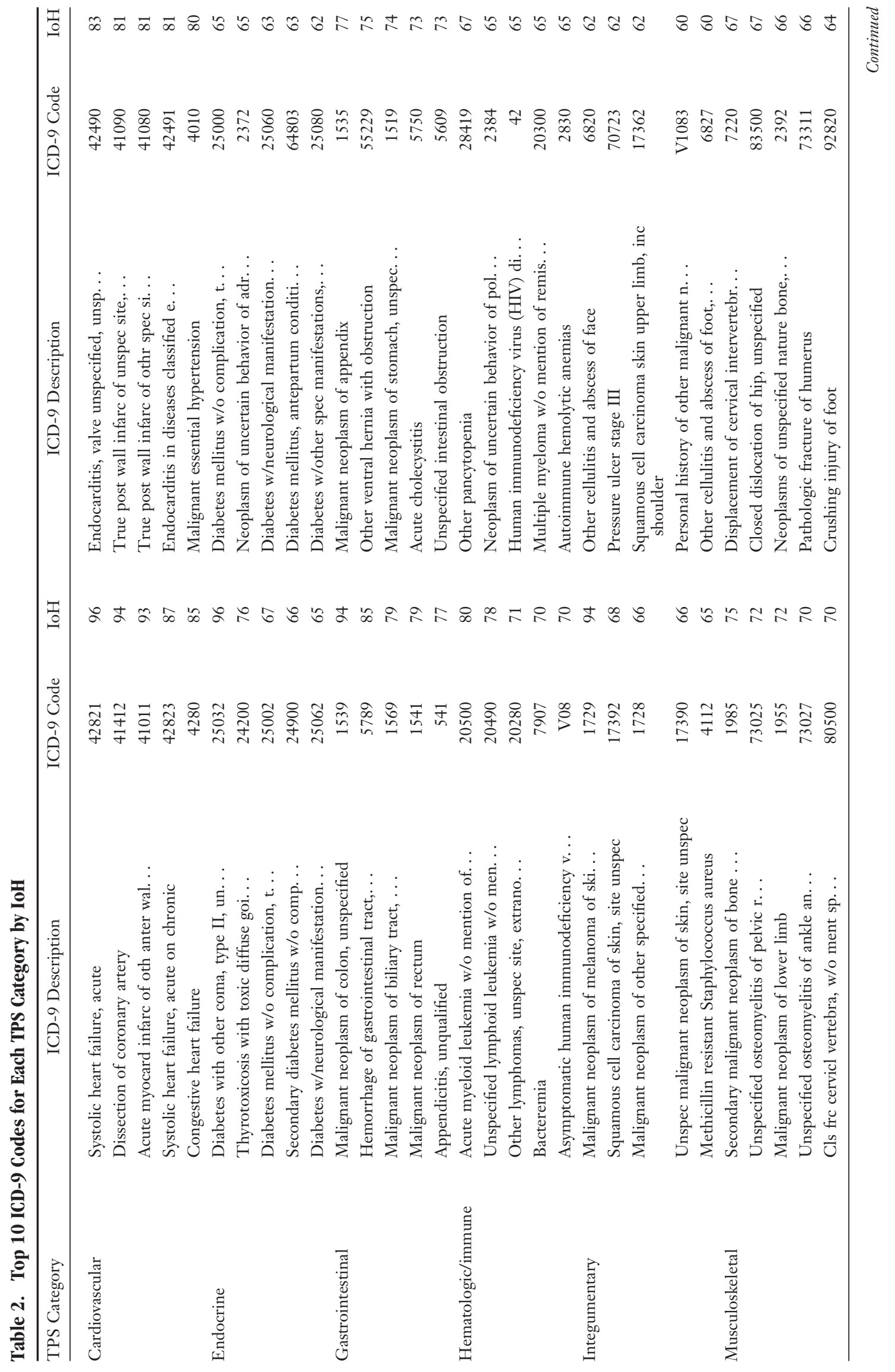




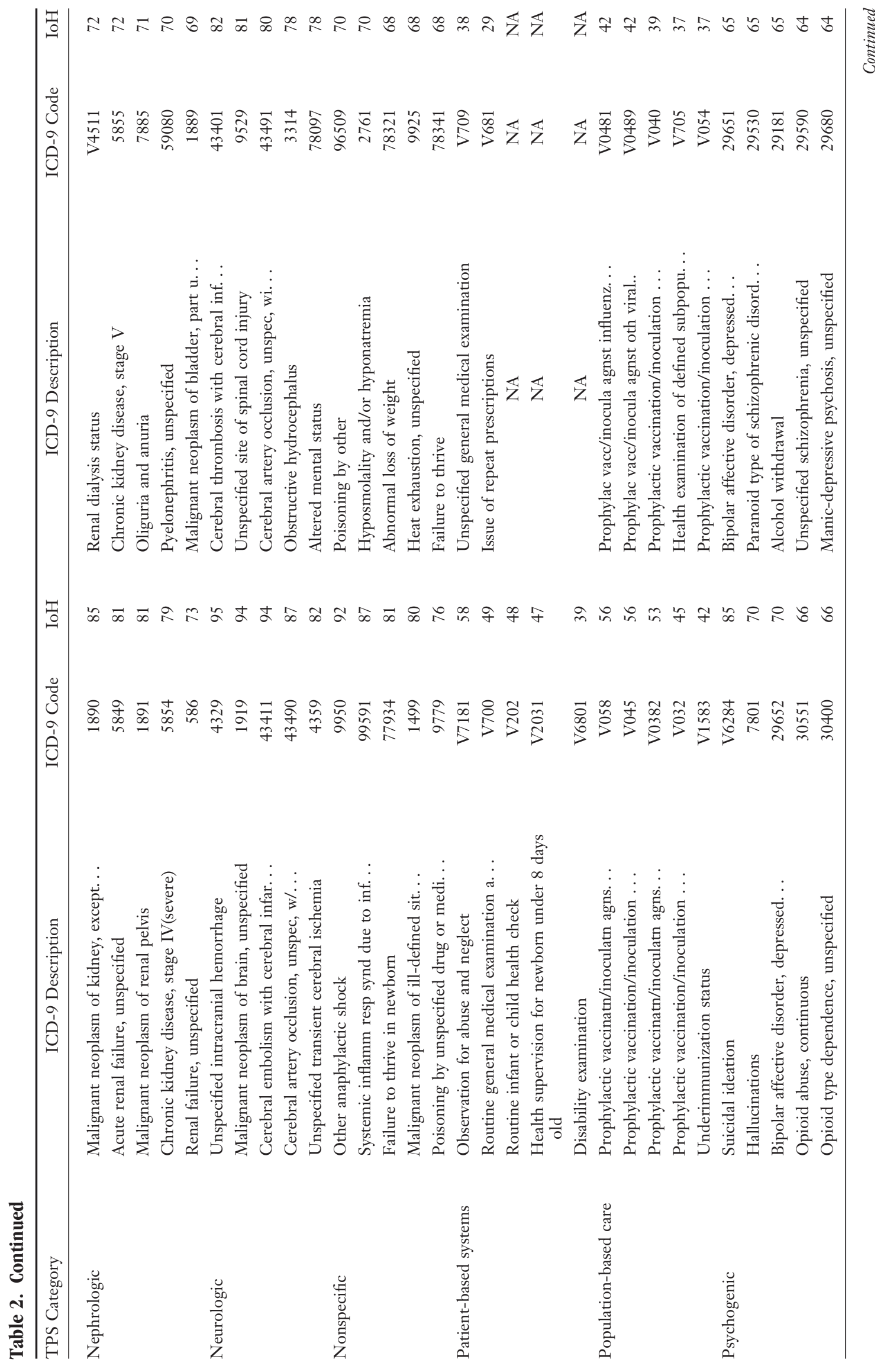




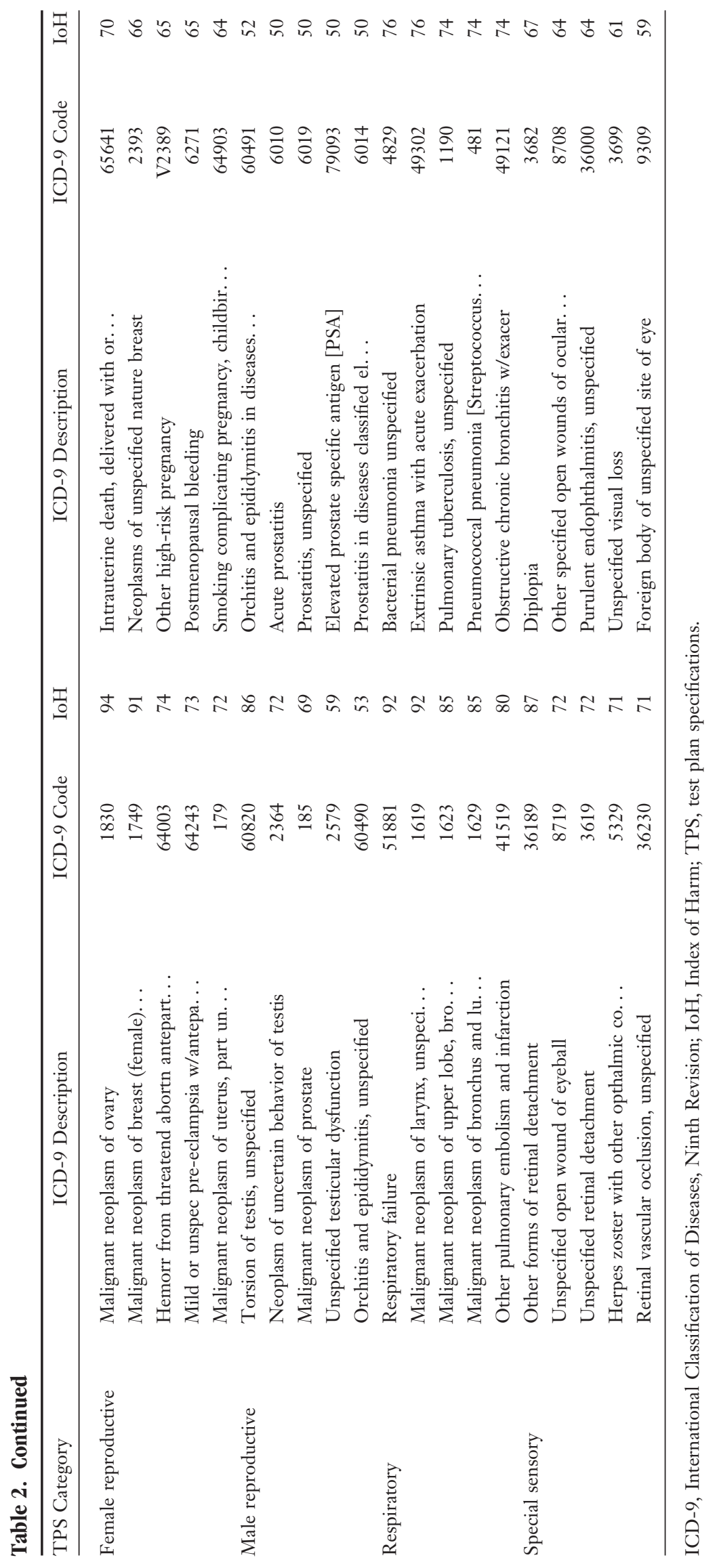


This was not a hypothesis testing study but rather a descriptive study that looked at the scope of what family physicians are doing in an ambulatory care setting from the perspectives of frequency and potential for patient harm. There is a subtle difference between what family physicians are doing and what the specialty of family medicine considers to be within its purview. This study considers only what family physicians are doing and is silent with regard to what is considered to be within the realm of family medicine. Nevertheless, this information is critical to anyone who is committed to preparing physicians to enter the specialty of family medicine, to providing relevant continuing medical education for them, or to accurately assessing their clinical knowledge. It is crucial that family medicine certification boards, both osteopathic or allopathic, understand what family physicians are doing, so that their certification process can sufficiently reflect the full range of the scope of family medicine. It is our hope that these descriptions can help the professional community identify appropriate content for teaching and testing the important aspects of family medicine.

The authors thank all the reviewers who helped to provide ratings: Steven Anderson, Elizabeth Baxley, John Beecher, Mark Bixby, John Brady, David Bucher, Colleen Conry, Stephen Craane, Christopher Cunha, Amy Daleiden, Tara Doyle-Bizily, Roger Fain, Patricia Fontaine, Joel Gordon, Joseph Gravel, Manuel Idrogo, Linda Johnson, Sandeep Kalola, Steven Kind, Jerry Kruse, Christine Matson, Mrinalini Mudkanna, Shannon Neale, Stephen Ready, David Satin, David Schneider, Scott Staples, Philip Stoyke, and Mark Westholder.

To see this article online, please go to: bttp://jabfm.org/content/ 31/1/126.full.

\section{References}

1. Starfield B. Primary Care. New York, NY: Oxford University Press; 1998.

2. Accreditation Council for Graduate Medical Education. ACGME Program Requirements for Graduate Medical Education in Family Medicine. Chicago, IL: Accreditation Council for Graduate Medical Education; July 1, 2017.

3. Norris TE, Rovinelli RJ, Puffer JC, Rinaldo J, Price DW. From specialty-based to practice-based: A new blueprint for the American Board of Family Medicine cognitive examination. J Am Board Fam Pract 2005;18:546-54.

4. Tong ST, Makaroff LA, Xierali IM, et al. Proportion of family physicians providing maternity care con- tinues to decline. J Am Board Fam Med 2012;25: $270-1$.

5. Xierali IM, Puffer JC, Tong ST, Bazemore AW, Green LA. The percentage of family physicians attending to women's gender-specific health needs is declining. J Am Board Fam Med 2012;25:406-7.

6. Bazemore AW, Makaroff LA, Puffer JC, et al. Declining numbers of family physicians are caring for children. J Am Board Fam Med 2012;25:139-40.

7. Tversky A, Kahneman D. Availability: A heuristic for judging frequency and probability. Cogn Psychol 1973;5:207-32.

8. Kahneman D, Tversky A. Subjective probability: A judgement of representativeness. Cogn Psychol 1972;3:430-54.

9. National Center for Health Statistics. National Center for Health Statistics Ambulatory Health Care Data. 2012.

10. U.S. Department of Health and Human Services. Centers for Disease Control and Prevention, Centers for Medicare and Medicaid Services. International classification of diseases, ninth revision, clinical modification. DHHS Pub No. (PHS) 061260.2006.

11. Xierali IM, Rinaldo JC, Green LA, et al. Family physician participation in maintenance of certification. Ann Fam Med 2011;9:203-10.

12. National Center for Health Statistics. NAMCS 2012 micro-data file documentation. 2012.

13. Linacre JM. Disconnected subsets, Guttman patterns and data connectivity. Rasch Meas Transact 2013;27:1415-7.

14. Linacre JM. Judging plans and facets. Research Note 3 MESA Psychometric Laboratory. Chicago, IL: University of Chicago; 1997.

15. Rasch G. Probabilistic models for some intelligence and attainment tests. Copenhagen, Denmark: Danish Institute for Educational Research; 1960.

16. Andrich D. A rating formulation for ordered response categories. Psychometrika 1978;43:561-73.

17. Wright BD, Masters GN. Rating scale analysis. Chicago, IL: MESA Press; 1982.

18. Smith RM. Fit analysis in latent trait measurement models. J Appl Meas 2000;1:199-218.

19. Linacre JM. What do infit and outfit, mean-square and standardized mean? Rasch Meas Transact 2002; 16:878.

20. Smith EV. Evidence for the reliability of measures and validity of measure interpretation: A Rasch measurement perspective. In: Smith EV, Smith RM, eds. Introduction to Rasch measurement. Maple Grove, MN: JAM Press; 2004, 93-122.

21. Andrich D. Measurement criteria for choosing among models for graded responses. In: von Eye A, Clogg CC, eds. Analysis of categorical variables in developmental research. Orlando, FL: Academic Press; 1996, 3-35. 


\begin{tabular}{|c|c|c|c|c|c|c|}
\hline Item & ICD-9 Description & ICD-9 Code & $\begin{array}{l}\text { Weighted } \\
\text { Frequency }\end{array}$ & $\begin{array}{c}\text { Decision } \\
\text { Round }\end{array}$ & TPS Category & $\mathrm{IoH}$ \\
\hline 1 & Unspecified anomaly of upper limb & 75550 & 3298 & $\mathrm{MN}$ & Musculoskeletal & 34 \\
\hline 2 & Other symptoms involving respiratory ... & 7869 & 337213 & $\mathrm{MN}$ & Respiratory & 43 \\
\hline 3 & Unspecified internal derangement of knee & 7179 & 232641 & $\mathrm{MN}$ & Musculoskeletal & 48 \\
\hline 4 & Common migraine without mention of in. . & 34610 & 49001 & $\mathrm{MN}$ & Neurologic & 53 \\
\hline 5 & Aggressive periodontitis, localized & 52331 & 28430 & $\mathrm{EC}$ & Special.Sensory & 40 \\
\hline 6 & Herpes zoster without mention of comp... & 539 & 682125 & $\mathrm{MN}$ & Integumentary & 45 \\
\hline 7 & End stage renal disease & 5856 & 86655 & $\mathrm{MN}$ & Nephrologic & 68 \\
\hline 8 & Menopausal or female climacteric states & 6272 & 392353 & $\mathrm{MN}$ & Reproductive.Female & 33 \\
\hline 10 & Ulcerative colitis, unspecified & 5569 & 73371 & $\mathrm{MN}$ & Gastrointestinal & 56 \\
\hline 12 & Arthropathy, unspecified, site unspec. . & 71690 & 820981 & $\mathrm{MN}$ & Musculoskeletal & 39 \\
\hline 13 & Other inflammatory disorders of penis & 6072 & 46806 & $\mathrm{MN}$ & Reproductive.Male & 45 \\
\hline 14 & Spinal stenosis, unspecified region & 72400 & 183216 & $\mathrm{MN}$ & Musculoskeletal & 56 \\
\hline 15 & Acute serous otitis media & 38101 & 210637 & $\mathrm{MN}$ & Special.Sensory & 36 \\
\hline 17 & Unspecified disorder of urethra and u. . . & 5999 & 59827 & $\mathrm{EC}$ & Nephrologic & 38 \\
\hline 18 & Head injury, unspecified & 95901 & 46198 & $\mathrm{MN}$ & Neurologic & 75 \\
\hline 19 & Alcoholic liver damage, unspecified & 5713 & 21003 & $\mathrm{MN}$ & Gastrointestinal & 63 \\
\hline 20 & Perforation of tympanic membrane, uns. . & 38420 & 44707 & $\mathrm{MN}$ & Special.Sensory & 40 \\
\hline 21 & Mylagia and myositis, unspecified & 7291 & 1675537 & $\mathrm{MN}$ & Musculoskeletal & 42 \\
\hline 22 & Unspecified cataract & 3669 & 196135 & $\mathrm{MN}$ & Special.Sensory & 39 \\
\hline 23 & Varicose veins of lower extremities wi.. & 4541 & 53386 & $\mathrm{MN}$ & Cardiovascular & 27 \\
\hline 24 & Acute upper respiratory infections of. . & 4658 & 115457 & $\mathrm{MN}$ & Respiratory & 38 \\
\hline 25 & Foreign body in conjunctival sac & 9301 & 28430 & $\mathrm{MN}$ & Special.Sensory & 42 \\
\hline 26 & Trigger finger (acquired) & 72703 & 64711 & $\mathrm{MN}$ & Musculoskeletal & 24 \\
\hline 27 & Trichomonal vulvovaginitis & 13101 & 32701 & $\mathrm{MN}$ & Reproductive.Female & 51 \\
\hline 28 & Other specified diseases of sebaceous... & 7068 & 37630 & $\mathrm{MN}$ & Integumentary & 16 \\
\hline 31 & Other chronic pulmonary heart diseases & 4168 & 54208 & $\mathrm{MN}$ & Cardiovascular & 63 \\
\hline 32 & Infectious diarrhea & 92 & 4839 & $\mathrm{MN}$ & Gastrointestinal & 51 \\
\hline 33 & Open wound(s) (multiple) of unspecifi... & 8799 & 17385 & $\mathrm{MN}$ & Integumentary & 47 \\
\hline 34 & Trigeminal neuralgia & 3501 & 166042 & $\mathrm{MN}$ & Neurologic & 53 \\
\hline 36 & Thrombocytopenia, unspecified & 2875 & 71118 & $\mathrm{MN}$ & Hematologic.Immune & 53 \\
\hline 37 & Other specified headache syndromes & 33989 & 11609 & $\mathrm{MN}$ & Neurologic & 51 \\
\hline 38 & Female infertility of unspecified origin & 6289 & 1739 & $\mathrm{MN}$ & Reproductive.Female & 47 \\
\hline 39 & Other pancytopenia & 28419 & 1830 & $\mathrm{MN}$ & Hematologic.Immune & 67 \\
\hline 40 & Unspecified otitis media & 3829 & 1940521 & $\mathrm{MN}$ & Special.Sensory & 44 \\
\hline 42 & Nephrotic syndrome with unspecif path. . . & 5819 & 11463 & $\mathrm{MN}$ & Nephrologic & 65 \\
\hline 43 & Pulmonary congestion and hypostasis & 514 & 6331 & $\mathrm{MN}$ & Respiratory & 67 \\
\hline 44 & Chronic ischemic heart disease, unspe... & 4149 & 38355 & $\mathrm{MN}$ & Cardiovascular & 68 \\
\hline 45 & Personal history of poliomyelitis & V1202 & 29838 & $\mathrm{MN}$ & Neurologic & 43 \\
\hline 46 & Mild cognitive impairment, so stated & 33183 & 193805 & $\mathrm{MN}$ & Neurologic & 42 \\
\hline 47 & Diabetes w/other spec manifestations,. . & 25080 & 84554 & $\mathrm{MN}$ & Endocrine & 62 \\
\hline 48 & Dysuria & 7881 & 921321 & $\mathrm{EC}$ & Nephrologic & 42 \\
\hline 50 & Other ill-defined disorders of eye & 37999 & 53077 & $\mathrm{MN}$ & Special.Sensory & 40 \\
\hline 51 & Diagnosis of 'none' & V997 & 695957 & $\mathrm{MN}$ & Nonspecific & 3 \\
\hline 52 & Extrinsic asthma without mention of s... & 49300 & 259297 & $\mathrm{MN}$ & Respiratory & 53 \\
\hline 53 & Abscess of eyelid & 37313 & 17594 & $\mathrm{EC}$ & Special.Sensory & 45 \\
\hline 54 & Sprains and strains of lumbar & 8472 & 1127251 & $\mathrm{MN}$ & Musculoskeletal & 42 \\
\hline
\end{tabular}




\begin{tabular}{|c|c|c|c|c|c|c|}
\hline Item & ICD-9 Description & ICD-9 Code & $\begin{array}{l}\text { Weighted } \\
\text { Frequency }\end{array}$ & $\begin{array}{c}\text { Decision } \\
\text { Round }\end{array}$ & TPS Category & $\mathrm{IoH}$ \\
\hline 55 & Infectious colitis, enteritis, and ga... & 90 & 63130 & $\mathrm{MN}$ & Gastrointestinal & 51 \\
\hline 57 & Other penile anomalies & 75269 & 4811 & $\mathrm{MN}$ & Reproductive.Male & 27 \\
\hline 58 & Nausea alone & 78702 & 802891 & $\mathrm{MN}$ & Gastrointestinal & 33 \\
\hline 59 & Senile osteoporosis & 73301 & 33516 & $\mathrm{MN}$ & Musculoskeletal & 48 \\
\hline 60 & Family history of arthritis & V177 & 1522 & $\mathrm{MN}$ & Musculoskeletal & 13 \\
\hline 61 & Benign local hyper prost w/o urin obs. . . & 60020 & 5946 & $\mathrm{MN}$ & Reproductive.Male & 45 \\
\hline 62 & Food poisoning, unspecified & 59 & 1830 & $\mathrm{MN}$ & Gastrointestinal & 42 \\
\hline 64 & Unspecified disorder of gallbladder & 5759 & 18419 & $\mathrm{MN}$ & Gastrointestinal & 48 \\
\hline 65 & Psychogenic vaginismus & 30651 & 15464 & $\mathrm{EC}$ & Psychogenic & 36 \\
\hline 66 & Acute posthemorrhagic anemia & 2851 & 9009 & $\mathrm{MN}$ & Hematologic.Immune & 56 \\
\hline 67 & Secondary to blood loss (chronic) & 2800 & 21451 & $\mathrm{MN}$ & Hematologic.Immune & 45 \\
\hline 68 & Nonallopathic lesions, thoracic region & 7392 & 268000 & $\mathrm{MN}$ & Musculoskeletal & 30 \\
\hline 70 & Other dermatitis or eczema due to oth... & 69289 & 7828 & $\mathrm{MN}$ & Integumentary & 30 \\
\hline 71 & Edema & 7823 & 1238728 & $\mathrm{MN}$ & Cardiovascular & 51 \\
\hline 72 & Concussion, loss of consciousness uns. . & 8509 & 76361 & $\mathrm{MN}$ & Neurologic & 72 \\
\hline 73 & Benign hypertensive heart disease wit... & 40210 & 19857 & $\mathrm{MN}$ & Cardiovascular & 53 \\
\hline 74 & Umbilical hernia & 5531 & 97615 & $\mathrm{MN}$ & Gastrointestinal & 27 \\
\hline 75 & Unspecified osteomyelitis of pelvic r... & 73025 & 28430 & $\mathrm{MN}$ & Musculoskeletal & 72 \\
\hline 76 & Routine postpartum follow-up & V242 & 207901 & $\mathrm{MN}$ & Reproductive.Female & 30 \\
\hline 77 & Unspecified adverse effect of other drug... & 99529 & 2948 & $\mathrm{MN}$ & Nonspecific & 43 \\
\hline 78 & Hyperhidrosis & 7808 & 66779 & $\mathrm{MN}$ & Integumentary & 21 \\
\hline 79 & Schizo-affective type of schizophreni. .. & 29570 & 23114 & $\mathrm{MN}$ & Psychogenic & 55 \\
\hline 80 & Chronic ulcer of unspecified site & 7079 & 51706 & $\mathrm{MN}$ & Integumentary & 51 \\
\hline 81 & Clos fract lumbar vertebrae, w/o ment... & 8054 & 7998 & $\mathrm{MN}$ & Musculoskeletal & 56 \\
\hline 82 & Disturbance of skin sensation & 7820 & 493688 & $\mathrm{MN}$ & Neurologic & 33 \\
\hline 83 & Closed fracture of upper end of humer. . & 81200 & 6911 & $\mathrm{MN}$ & Musculoskeletal & 53 \\
\hline 84 & Gastroenteritis and colitis due to ra... & 5581 & 10250 & $\mathrm{MN}$ & Gastrointestinal & 48 \\
\hline 85 & Hepatomegaly & 7891 & 24621 & $\mathrm{MN}$ & Gastrointestinal & 48 \\
\hline 86 & Vestibular neuronitis & 38612 & 7355 & $\mathrm{EC}$ & Special.Sensory & 48 \\
\hline 88 & Unspecified circulatory system disorder & 4599 & 45866 & $\mathrm{MN}$ & Cardiovascular & 42 \\
\hline 89 & Eustachian salpingitis, unspecified & 38150 & 28430 & $\mathrm{MN}$ & Special.Sensory & 41 \\
\hline 90 & Postcoital bleeding & 6267 & 22796 & $\mathrm{MN}$ & Reproductive.Female & 42 \\
\hline 91 & Osteoarthrosis, localized, primary, p... & 71515 & 28430 & $\mathrm{MN}$ & Musculoskeletal & 33 \\
\hline 92 & Other dyspnea and respiratory abnorma... & 78609 & 928862 & $\mathrm{MN}$ & Respiratory & 60 \\
\hline 93 & Personal history of pneumonia & V1261 & 7519 & $\mathrm{MN}$ & Respiratory & 30 \\
\hline 94 & Pityriasis rosea & 6963 & 4070 & $\mathrm{MN}$ & Integumentary & 27 \\
\hline 95 & Postlaminectomy syndrome, lumbar region & 72283 & 44226 & $\mathrm{MN}$ & Musculoskeletal & 42 \\
\hline 97 & Posttraumatic wound infection, not el. . & 9583 & 6783 & $\mathrm{MN}$ & Integumentary & 55 \\
\hline 98 & Cardiac pacemaker & V4501 & 44241 & $\mathrm{MN}$ & Cardiovascular & 47 \\
\hline 100 & Unspecified derangement of joint of p... & 71895 & 46806 & $\mathrm{MN}$ & Musculoskeletal & 37 \\
\hline 101 & Cluster headache syndrome, unspecified & 33900 & 25374 & $\mathrm{MN}$ & Neurologic & 49 \\
\hline 103 & Contusion of elbow & 92311 & 6355 & $\mathrm{MN}$ & Musculoskeletal & 12 \\
\hline 104 & Myasthenia gravis w/o (acute) exacerb... & 35800 & 50474 & $\mathrm{MN}$ & Neurologic & 60 \\
\hline 105 & Malignant neoplasm of melanoma of ski... & 1729 & 35160 & $\mathrm{MN}$ & Integumentary & 94 \\
\hline 106 & Stiffness of joint of site unspecifie. . & 71950 & 59876 & $\mathrm{MN}$ & Musculoskeletal & 31 \\
\hline 107 & Other chronic postoperative pain & 33828 & 393702 & $\mathrm{EC}$ & Nonspecific & 43 \\
\hline 108 & Asymptomatic postmenopausal status (a. . & V4981 & 83992 & $\mathrm{MN}$ & Reproductive.Female & 6 \\
\hline 109 & Malignant neoplasm of brain, unspecified & 1919 & 11463 & $\mathrm{MN}$ & Neurologic & 94 \\
\hline 111 & Other threatened labor w/antepartum c... & 64413 & 17594 & $\mathrm{MN}$ & Reproductive.Female & 57 \\
\hline 112 & Abrasion or friction burn of hip/thig. . . & 9160 & 27478 & $\mathrm{MN}$ & Integumentary & 12 \\
\hline
\end{tabular}




\begin{tabular}{|c|c|c|c|c|c|c|}
\hline Item & ICD-9 Description & ICD-9 Code & $\begin{array}{l}\text { Weighted } \\
\text { Frequency }\end{array}$ & $\begin{array}{l}\text { Decision } \\
\text { Round }\end{array}$ & TPS Category & $\mathrm{IoH}$ \\
\hline 113 & Hyperemesis gravidarum w/metabol dist. . . & 64313 & 5192 & $\mathrm{MN}$ & Reproductive.Female & 62 \\
\hline 115 & Other affections of shoulder region, ... & 7262 & 101462 & $\mathrm{MN}$ & Musculoskeletal & 37 \\
\hline 116 & Atrial flutter & 42732 & 1981 & $\mathrm{MN}$ & Cardiovascular & 71 \\
\hline 117 & Acute gouty arthropathy & 27401 & 43625 & $\mathrm{MN}$ & Musculoskeletal & 52 \\
\hline 118 & Primary hypercoagulable state & 28981 & 11446 & $\mathrm{MN}$ & Hematologic.Immune & 60 \\
\hline 120 & Burn, unspecified, blisters, epiderma... & 9492 & 11219 & $\mathrm{MN}$ & Integumentary & 31 \\
\hline 122 & $\begin{array}{l}\text { Obstructive chronic bronchitis w/acute } \\
\text { bronchitis }\end{array}$ & 49122 & 40718 & $\mathrm{MN}$ & Respiratory & 68 \\
\hline 123 & Open wound of toe(s), without mention. . & 8930 & 26410 & $\mathrm{MN}$ & Integumentary & 39 \\
\hline 124 & Cerebral embolism with cerebral infar. . . & 43411 & 16002 & $\mathrm{MN}$ & Neurologic & 94 \\
\hline 125 & Narcolepsy without cataplexy & 34700 & 15220 & $\mathrm{MN}$ & Neurologic & 54 \\
\hline 126 & Acute bronchitis & 4660 & 2341367 & $\mathrm{MN}$ & Respiratory & 44 \\
\hline 127 & Ulcer of ankle, exc decubitus & 70713 & 29644 & $\mathrm{MN}$ & Integumentary & 42 \\
\hline 128 & Aseptic necrosis of bone, site unspec. . . & 73340 & 5656 & $\mathrm{MN}$ & Musculoskeletal & 62 \\
\hline 129 & Pure hyperglyceridemia & 2721 & 195620 & $\mathrm{EC}$ & Cardiovascular & 44 \\
\hline 130 & Other chronic cystitis & 5952 & 6911 & $\mathrm{EC}$ & Nephrologic & 48 \\
\hline 131 & Peripheral autonomic neuropathy in di. . & 3371 & 62131 & $\mathrm{MN}$ & Neurologic & 54 \\
\hline 132 & Unspecified affective psychosis & 29690 & 152257 & $\mathrm{MN}$ & Psychogenic & 62 \\
\hline 133 & Malignant neoplasm of ovary & 1830 & 34319 & $\mathrm{MN}$ & Reproductive.Female & 94 \\
\hline 134 & $\begin{array}{l}\text { Health supervision for newborn } 8 \text { to } 28 \text { days } \\
\text { old }\end{array}$ & V2032 & 175957 & $\mathrm{MN}$ & Nonspecific & 42 \\
\hline 135 & Slow transit constipation & 56401 & 46806 & $\mathrm{MN}$ & Gastrointestinal & 34 \\
\hline 136 & Atherosclerosis of renal artery & 4401 & 8944 & EC & Nephrologic & 54 \\
\hline 137 & Other, mixed, or unspecified drug abu... & 30590 & 91929 & $\mathrm{MN}$ & Psychogenic & 60 \\
\hline 138 & Unspecified inflammation of eyelid & 3739 & 15474 & $\mathrm{MN}$ & Special.Sensory & 26 \\
\hline 139 & Pain in joint of other specified sites & 71948 & 20924 & $\mathrm{MN}$ & Musculoskeletal & 32 \\
\hline 140 & Osteoarthrosis, localized, primary, $1 . \ldots$ & 71516 & 101821 & $\mathrm{MN}$ & Musculoskeletal & 39 \\
\hline 141 & Chronic serous otitis media, simple o... & 38110 & 37630 & $\mathrm{MN}$ & Special.Sensory & 39 \\
\hline 142 & Adult emotional/psychological abuse & 99582 & 7437 & $\mathrm{MN}$ & Psychogenic & 60 \\
\hline 143 & Sprains and strains of neck & 8470 & 706793 & $\mathrm{MN}$ & Musculoskeletal & 37 \\
\hline 144 & Sprains and strains of unspecified si... & 8449 & 242161 & $\mathrm{MN}$ & Musculoskeletal & 31 \\
\hline 145 & Somnambulism or night terrors & 30746 & 17094 & $\mathrm{MN}$ & Psychogenic & 37 \\
\hline 146 & Oliguria and anuria & 7885 & 5798 & $\mathrm{MN}$ & Nephrologic & 71 \\
\hline 147 & Classical migraine with intractable $\mathrm{m} . .$. & 34601 & 9344 & $\mathrm{MN}$ & Neurologic & 60 \\
\hline 148 & Postinflammatory pulmonary fibrosis & 515 & 53921 & $\mathrm{MN}$ & Respiratory & 60 \\
\hline 150 & Arthropathy, unspecified, multiple sites & 71699 & 121679 & $\mathrm{MN}$ & Musculoskeletal & 37 \\
\hline 151 & $\begin{array}{l}\text { Squamous cell carcinoma skin lower limb, } \\
\text { inc hip }\end{array}$ & 17372 & 22341 & $\mathrm{MN}$ & Integumentary & 57 \\
\hline 153 & Sprains and strains of metatarsophala... & 84512 & 8698 & $\mathrm{MN}$ & Musculoskeletal & 28 \\
\hline 155 & Prostatocystitis & 6013 & 61744 & $\mathrm{MN}$ & Reproductive.Male & 49 \\
\hline 157 & Unspecified iridocyclitis & 3643 & 27114 & $\mathrm{MN}$ & Special.Sensory & 52 \\
\hline 158 & Circumscribed scleroderma & 7010 & 20739 & $\mathrm{MN}$ & Integumentary & 44 \\
\hline 159 & Cestode infection, unspecified & 1239 & 2948 & $\mathrm{EC}$ & Gastrointestinal & 45 \\
\hline 160 & Tear of medial cartilage or meniscus ... & 8360 & 47238 & $\mathrm{MN}$ & Musculoskeletal & 42 \\
\hline 161 & Unspecified intestinal malabsorption & 5799 & 112048 & $\mathrm{MN}$ & Gastrointestinal & 52 \\
\hline 162 & Condyloma acuminatum & 7811 & 53605 & $\mathrm{MN}$ & Integumentary & 34 \\
\hline 163 & Otogenic pain & 38871 & 32930 & $\mathrm{MN}$ & Special.Sensory & 34 \\
\hline 164 & Gouty nephropathy, unspecified & 27410 & 14370 & $\mathrm{MN}$ & Nephrologic & 54 \\
\hline 165 & Unspecified lymphoid leukemia w/o men. . . & 20490 & 13034 & $\mathrm{MN}$ & Hematologic.Immune & 78 \\
\hline 166 & Unspecified disease of respiratory sy. .. & 5199 & 103500 & $\mathrm{MN}$ & Respiratory & 38 \\
\hline
\end{tabular}




\begin{tabular}{|c|c|c|c|c|c|c|}
\hline Item & ICD-9 Description & ICD-9 Code & $\begin{array}{l}\text { Weighted } \\
\text { Frequency }\end{array}$ & $\begin{array}{l}\text { Decision } \\
\text { Round }\end{array}$ & TPS Category & $\mathrm{IoH}$ \\
\hline 167 & Other atopic dermatitis and related c. . & 6918 & 224209 & $\mathrm{MN}$ & Integumentary & 31 \\
\hline 168 & Transient hypertension of preg w/ante. . & 64233 & 17594 & $\mathrm{MN}$ & Reproductive.Female & 47 \\
\hline 169 & Anxiety state, unspecified & 30000 & 6500798 & $\mathrm{MN}$ & Psychogenic & 57 \\
\hline 170 & Effusion of joint of upper arm & 71902 & 29838 & $\mathrm{MN}$ & Musculoskeletal & 42 \\
\hline 171 & Volume depletion, unspecified & 27650 & 5798 & $\mathrm{MN}$ & Cardiovascular & 57 \\
\hline 172 & Atrial fibrillation & 42731 & 1703903 & $\mathrm{MN}$ & Cardiovascular & 68 \\
\hline 173 & Chondromalacia & 73392 & 3051 & $\mathrm{MN}$ & Musculoskeletal & 39 \\
\hline 174 & Bipolar affective disorder, depressed. . . & 29651 & 11161 & $\mathrm{MN}$ & Psychogenic & 65 \\
\hline 175 & Dermatophytosis of other specified sites & 1108 & 22147 & $\mathrm{MN}$ & Integumentary & 25 \\
\hline 176 & Bariatric surgery status & V4586 & 38358 & EC & Nonspecific & 46 \\
\hline 178 & Tobacco use disorder, unspecified & 3051 & 1421592 & EC & Psychogenic & 62 \\
\hline 179 & Hemorrhage of gastrointestinal tract,. . . & 5789 & 9610 & $\mathrm{MN}$ & Gastrointestinal & 85 \\
\hline 180 & Other specified disorders of joint, $f .$. & 71983 & 7818 & $\mathrm{MN}$ & Musculoskeletal & 33 \\
\hline 181 & Visual field defect, unspecified & 36840 & 28430 & EC & Special.Sensory & 47 \\
\hline 183 & Ventral hernia, unspecified & 55320 & 162505 & $\mathrm{EC}$ & Gastrointestinal & 34 \\
\hline 184 & Other anxiety state & 30009 & 45816 & $\mathrm{MN}$ & Psychogenic & 47 \\
\hline 185 & Insect bite of oth/multipl/unspec sit. . . & 9195 & 89061 & $\mathrm{MN}$ & Integumentary & 23 \\
\hline 186 & Percutaneous transluminal coronary an... & V4582 & 10996 & $\mathrm{MN}$ & Cardiovascular & 73 \\
\hline 187 & Irritable colon & 5641 & 364100 & $\mathrm{MN}$ & Gastrointestinal & 42 \\
\hline 188 & Carbuncle and furuncle of hand & 6804 & 6501 & $\mathrm{MN}$ & Integumentary & 37 \\
\hline 189 & Unspecified osteochondropathy & 7329 & 11398 & $\mathrm{MN}$ & Musculoskeletal & 37 \\
\hline 190 & Other specified menopausal and postme... & 6278 & 54628 & $\mathrm{MN}$ & Reproductive.Female & 43 \\
\hline 192 & Personal history of malignant neoplas. . . & V103 & 39221 & $\mathrm{EC}$ & Reproductive.Female & 53 \\
\hline 193 & Postmenopausal atrophic vaginitis & 6273 & 131700 & $\mathrm{MN}$ & Reproductive.Female & 31 \\
\hline 194 & Congestive heart failure & 4280 & 746334 & $\mathrm{MN}$ & Cardiovascular & 85 \\
\hline 195 & Renal colic & 7880 & 35860 & $\mathrm{MN}$ & Nephrologic & 60 \\
\hline 197 & $\begin{array}{l}\text { Unspecified viral hepatitis } \mathrm{C} \text { w/o hepatic } \\
\text { coma }\end{array}$ & 7070 & 212175 & $\mathrm{MN}$ & Gastrointestinal & 69 \\
\hline 198 & Opn wound of knee/leg [except thigh]/. . & 8910 & 167820 & $\mathrm{MN}$ & Integumentary & 47 \\
\hline 200 & Personal history of alcoholism & V113 & 3185 & $\mathrm{MN}$ & Psychogenic & 54 \\
\hline 201 & Personal history of other mental diso... & V118 & 7355 & $\mathrm{MN}$ & Psychogenic & 44 \\
\hline 202 & Other specified hypotension & 4588 & 29644 & $\mathrm{MN}$ & Cardiovascular & 60 \\
\hline 204 & Enthesopathy of elbow, unspecified & 72630 & 4201 & $\mathrm{MN}$ & Musculoskeletal & 24 \\
\hline 205 & Aphasia & 7843 & 44536 & $\mathrm{MN}$ & Neurologic & 66 \\
\hline 206 & Unspecified vertiginous syndromes and. . & 3869 & 24223 & $\mathrm{EC}$ & Neurologic & 52 \\
\hline 207 & Corns and callosities & 700 & 94364 & $\mathrm{MN}$ & Integumentary & 11 \\
\hline 208 & Malignant neoplasm of upper lobe, bro... & 1623 & 32442 & $\mathrm{MN}$ & Respiratory & 85 \\
\hline 209 & Respiratory abnormality, unspecified & 78600 & 93612 & $\mathrm{MN}$ & Respiratory & 37 \\
\hline 210 & Contracture of palmar fascia & 7286 & 19314 & $\mathrm{MN}$ & Musculoskeletal & 27 \\
\hline 211 & Unspecif nonpsychotic mentl disordr f. . . & 3109 & 28430 & $\mathrm{MN}$ & Psychogenic & 49 \\
\hline 212 & Dysphonia & 78442 & 73951 & $\mathrm{EC}$ & Respiratory & 50 \\
\hline 213 & Synovitis and tenosynovitis, unspecified & 72700 & 97397 & $\mathrm{MN}$ & Musculoskeletal & 39 \\
\hline 214 & Opioid abuse, continuous & 30551 & 150519 & $\mathrm{MN}$ & Psychogenic & 66 \\
\hline 215 & Legal blindness, as defined in U.S.A. & 3694 & 28430 & $\mathrm{MN}$ & Special.Sensory & 55 \\
\hline 216 & Malignant neoplasm of kidney, except ... & 1890 & 80925 & $\mathrm{MN}$ & Nephrologic & 85 \\
\hline 217 & $\begin{array}{l}\text { Smoking complicating pregnancy, } \\
\text { childbir... }\end{array}$ & 64903 & 17677 & $\mathrm{EC}$ & Reproductive.Female & 64 \\
\hline 218 & Osteoarthrosis mor thn 1 site, not sp... & 71589 & 37630 & $\mathrm{MN}$ & Musculoskeletal & 39 \\
\hline 220 & Seborrhea & 7063 & 30911 & $\mathrm{MN}$ & Integumentary & 18 \\
\hline
\end{tabular}




\begin{tabular}{|c|c|c|c|c|c|c|}
\hline Item & ICD-9 Description & ICD-9 Code & $\begin{array}{l}\text { Weighted } \\
\text { Frequency }\end{array}$ & $\begin{array}{l}\text { Decision } \\
\text { Round }\end{array}$ & TPS Category & $\mathrm{IoH}$ \\
\hline 221 & Pain in limb & 7295 & 2577512 & $\mathrm{MN}$ & Musculoskeletal & 37 \\
\hline 223 & Other conditions due to chromosome an... & 75889 & 8187 & $\mathrm{MN}$ & Nonspecific & 56 \\
\hline 224 & Drug withdrawal syndrome & 2920 & 48172 & $\mathrm{EC}$ & Nonspecific & 59 \\
\hline 225 & Influenza due to ident avian influ virus. . . & 48802 & 11887 & $\mathrm{MN}$ & Respiratory & 57 \\
\hline 226 & Other acquired deformities of toe & 7358 & 13628 & $\mathrm{MN}$ & Musculoskeletal & 19 \\
\hline 228 & Intestinal infection due to E. coli, & 800 & 28430 & $\mathrm{MN}$ & Gastrointestinal & 57 \\
\hline 229 & $\begin{array}{l}\text { Antineoplastic chemotherapy induced } \\
\text { anemia }\end{array}$ & 2853 & 3798 & $\mathrm{MN}$ & Hematologic.Immune & 61 \\
\hline 230 & Flatulence, eructation, and gas pain & 7873 & 64484 & $\mathrm{MN}$ & Gastrointestinal & 21 \\
\hline 231 & Partial tear of rotator cuff & 72613 & 16002 & $\mathrm{MN}$ & Musculoskeletal & 48 \\
\hline 232 & Infec/inflam reac due to oth genitour. . & 99665 & 27114 & $\mathrm{EC}$ & Nephrologic & 45 \\
\hline 233 & Pruritus ani & 6980 & 10574 & $\mathrm{MN}$ & Gastrointestinal & 21 \\
\hline 234 & Other specified anomalies & 75989 & 6228 & $\mathrm{MN}$ & Nonspecific & 34 \\
\hline 235 & Nonallopathic lesions, cervical region & 7391 & 222095 & $\mathrm{MN}$ & Musculoskeletal & 37 \\
\hline 237 & Unspecified derangement of joint of o... & 71898 & 14372 & $\mathrm{MN}$ & Musculoskeletal & 42 \\
\hline 238 & Keratoderma, acquired & 7011 & 51718 & $\mathrm{MN}$ & Integumentary & 24 \\
\hline 239 & Rheumatoid arthritis & 7140 & 480534 & $\mathrm{MN}$ & Musculoskeletal & 61 \\
\hline 240 & Unspec hypertensive renal disease w/o. . . & 40390 & 174204 & $\mathrm{EC}$ & Nephrologic & 61 \\
\hline 241 & Personal history of other specified diseases & V1389 & 8368 & $\mathrm{MN}$ & Nonspecific & 33 \\
\hline 242 & Restless legs syndrome (RLS) & 33394 & 391944 & $\mathrm{MN}$ & Neurologic & 41 \\
\hline 243 & Polyneuropathy due to other toxic agents & 3577 & 3798 & $\mathrm{MN}$ & Neurologic & 57 \\
\hline 244 & Major depressive disorder, single epi. . & 29621 & 3185 & $\mathrm{MN}$ & Psychogenic & 61 \\
\hline 245 & Impaired fasting glucose & 79021 & 316804 & $\mathrm{MN}$ & Endocrine & 50 \\
\hline 246 & Symptomatic inflammatory myopathy in ... & 3596 & 15811 & $\mathrm{MN}$ & Musculoskeletal & 50 \\
\hline 247 & Adjustment reaction with mixed emotio. . . & 30928 & 86650 & $\mathrm{MN}$ & Psychogenic & 48 \\
\hline 248 & Other specified disorders of eye and ... & 3798 & 8286 & $\mathrm{MN}$ & Special.Sensory & 37 \\
\hline 249 & Closed fracture of phalanx or phalang. . . & 81600 & 64001 & $\mathrm{MN}$ & Musculoskeletal & 45 \\
\hline 250 & Fracture of unspecified bone, closed & 8290 & 65368 & $\mathrm{MN}$ & Musculoskeletal & 44 \\
\hline 251 & Unspecified disorder of ear & 3889 & 4159 & $\mathrm{MN}$ & Special.Sensory & 37 \\
\hline 252 & Spontaneous ecchymoses & 7827 & 50277 & $\mathrm{MN}$ & Hematologic.Immune & 45 \\
\hline 254 & Unspecified hemorrhoids with other co. . . & 4558 & 46622 & $\mathrm{EC}$ & Gastrointestinal & 39 \\
\hline 255 & Anemia in neoplastic disease & 28522 & 14090 & $\mathrm{MN}$ & Hematologic.Immune & 61 \\
\hline 256 & Contusion of wrist & 92321 & 58646 & $\mathrm{MN}$ & Musculoskeletal & 18 \\
\hline 257 & Stress fracture of the metatarsals & 73394 & 23928 & $\mathrm{MN}$ & Musculoskeletal & 48 \\
\hline 258 & Sprains and strains of unspecified site & 84200 & 183770 & $\mathrm{MN}$ & Musculoskeletal & 29 \\
\hline 259 & Cervicalgia & 7231 & 2434661 & $\mathrm{MN}$ & Musculoskeletal & 41 \\
\hline 260 & Unspecified disorder of skin and subc. . . & 7099 & 665025 & $\mathrm{MN}$ & Integumentary & 29 \\
\hline 261 & Pain in joint of multiple sites & 71949 & 308857 & $\mathrm{MN}$ & Musculoskeletal & 43 \\
\hline 262 & Unspecified menopausal and postmenopa. . . & 6279 & 24179 & $\mathrm{MN}$ & Reproductive.Female & 39 \\
\hline 263 & Postconcussion syndrome & 3102 & 120675 & $\mathrm{MN}$ & Neurologic & 66 \\
\hline 264 & Prophylac vacc/inocu agnst diphtheria. . . & V063 & 5656 & $\mathrm{EC}$ & Nonspecific & 44 \\
\hline 265 & Contusion of foot & 92420 & 246141 & $\mathrm{MN}$ & Musculoskeletal & 18 \\
\hline 266 & Neoplasms of unspecified nature breast & 2393 & 4074 & $\mathrm{EC}$ & Reproductive.Female & 66 \\
\hline 267 & Other cellulitis and abscess of neck & 6821 & 33857 & $\mathrm{MN}$ & Integumentary & 52 \\
\hline 268 & Other specified endocrine disorders & 2598 & 46806 & $\mathrm{MN}$ & Endocrine & 42 \\
\hline 270 & Open wound of ear, part unspecif, w/o... & 8728 & 28430 & $\mathrm{EC}$ & Special.Sensory & 36 \\
\hline 271 & Malignant neoplasm of bronchus and lu. . . & 1629 & 122609 & $\mathrm{MN}$ & Respiratory & 85 \\
\hline 272 & Body Mass Index 45.0 to 49.9 , adult & V8542 & 37630 & $\mathrm{EC}$ & Nonspecific & 64 \\
\hline 273 & Arthropathy, unspecified, hand & 71694 & 11812 & $\mathrm{MN}$ & Musculoskeletal & 36 \\
\hline
\end{tabular}




\begin{tabular}{|c|c|c|c|c|c|c|}
\hline Item & ICD-9 Description & ICD-9 Code & $\begin{array}{l}\text { Weighted } \\
\text { Frequency }\end{array}$ & $\begin{array}{l}\text { Decision } \\
\text { Round }\end{array}$ & TPS Category & $\mathrm{IoH}$ \\
\hline 274 & Functional disturbances following car. . . & 4294 & 28430 & $\mathrm{EC}$ & Nonspecific & 45 \\
\hline 275 & Unspecified polyarthropathy or polyar. . & 71659 & 19803 & $\mathrm{MN}$ & Musculoskeletal & 48 \\
\hline 276 & Personal history of peptic ulcer disease & V1271 & 27114 & $\mathrm{MN}$ & Gastrointestinal & 39 \\
\hline 278 & Background diabetic retinopathy & 36201 & 23065 & $\mathrm{EC}$ & Special.Sensory & 57 \\
\hline 280 & Tibialis tendinitis & 72672 & 5126 & $\mathrm{MN}$ & Musculoskeletal & 29 \\
\hline 281 & Unspecified schizophrenia, unspecified & 29590 & 148188 & $\mathrm{MN}$ & Psychogenic & 64 \\
\hline 282 & Other reasons for seeking consultation & V658 & 44018 & $\mathrm{MN}$ & Nonspecific & 34 \\
\hline 283 & Malignant neoplasm of biliary tract, ... & 1569 & 4511 & $\mathrm{MN}$ & Gastrointestinal & 79 \\
\hline 284 & Other specified open wounds of ocular. . & 8708 & 1985 & $\mathrm{MN}$ & Special.Sensory & 64 \\
\hline 285 & Thoracic intervertebral disc without ... & 72211 & 93612 & $\mathrm{MN}$ & Musculoskeletal & 45 \\
\hline 286 & Ulcer oth part lower limbs, exc decubitus & 70719 & 3177 & $\mathrm{MN}$ & Integumentary & 41 \\
\hline 287 & Urinary obstruction, unspecified & 59960 & 29998 & EC & Nephrologic & 56 \\
\hline 288 & Attention to gastrostomy & V551 & 4419 & $\mathrm{MN}$ & Gastrointestinal & 29 \\
\hline 289 & Other laboratory examination & V7269 & 420909 & $\mathrm{MN}$ & Nonspecific & 21 \\
\hline 290 & Onychia and paronychia of toe & 68111 & 29987 & $\mathrm{MN}$ & Integumentary & 27 \\
\hline 291 & Chronic kidney disease, Stage II(mild) & 5852 & 90415 & $\mathrm{MN}$ & Nephrologic & 41 \\
\hline 292 & Unspecified organic brain syndrome (c. . . & 2949 & 82061 & $\mathrm{MN}$ & Neurologic & 59 \\
\hline 293 & Chronic tension type headache & 33912 & 9344 & $\mathrm{EC}$ & Neurologic & 45 \\
\hline 294 & Esophageal reflux & 53081 & 4623720 & $\mathrm{MN}$ & Gastrointestinal & 43 \\
\hline 295 & Transferred to another facility, sent... & V992 & 68278 & $\mathrm{MN}$ & Nonspecific & 40 \\
\hline 296 & Nocturnal enuresis & 78836 & 12477 & $\mathrm{EC}$ & Nephrologic & 39 \\
\hline 297 & Hyperventilation & 78601 & 17572 & $\mathrm{EC}$ & Respiratory & 42 \\
\hline 298 & Depressive disorder, not elsewhere cl... & 311 & 6011311 & $\mathrm{MN}$ & Psychogenic & 52 \\
\hline 299 & Pernicious anemia & 2810 & 119671 & $\mathrm{MN}$ & Hematologic.Immune & 52 \\
\hline 300 & Lyme disease & 8881 & 142347 & EC & Nonspecific & 64 \\
\hline 301 & Calculus of ureter & 5921 & 39797 & $\mathrm{EC}$ & Nephrologic & 63 \\
\hline 302 & Stricture and stenosis of esophagus & 5303 & 8118 & $\mathrm{MN}$ & Gastrointestinal & 58 \\
\hline 303 & Unspecified chronic bronchitis & 4919 & 53644 & $\mathrm{MN}$ & Respiratory & 51 \\
\hline 304 & Cholecystitis, unspecified & 57510 & 28061 & $\mathrm{MN}$ & Gastrointestinal & 55 \\
\hline 305 & Other cellulitis and abscess of leg, ... & 6826 & 413751 & $\mathrm{MN}$ & Integumentary & 49 \\
\hline 306 & Benign neoplasm of skin of other and ... & 2163 & 92853 & $\mathrm{MN}$ & Integumentary & 20 \\
\hline 307 & Abnormal involuntary movements & 7810 & 165627 & $\mathrm{MN}$ & Neurologic & 55 \\
\hline 308 & Examination following other surgery & V6709 & 215257 & $\mathrm{MN}$ & Nonspecific & 35 \\
\hline 309 & Cervical incompetence, antepartum con... & 65453 & 15464 & $\mathrm{MN}$ & Reproductive.Female & 60 \\
\hline 310 & Unequal leg length (acquired) & 73681 & 27114 & $\mathrm{MN}$ & Musculoskeletal & 33 \\
\hline 311 & Trans-sexualism with unspecified sexu. . . & 30250 & 5739 & EC & Psychogenic & 44 \\
\hline 312 & Meniere`s disease, unspecified & 38600 & 62180 & $\mathrm{MN}$ & Special.Sensory & 50 \\
\hline 313 & Other general symptoms & 78099 & 12457 & $\mathrm{MN}$ & Nonspecific & 26 \\
\hline 314 & Episodic tension type headache & 33911 & 6501 & $\mathrm{MN}$ & Neurologic & 41 \\
\hline 315 & Unspecified hereditary and idiopathic. . . & 3569 & 473502 & $\mathrm{EC}$ & Neurologic & 39 \\
\hline 316 & Observation for unspecified suspected... & V719 & 55611 & $\mathrm{MN}$ & Nonspecific & 23 \\
\hline 317 & Allergic rhinitis cause unspecified & 4779 & 3696363 & $\mathrm{EC}$ & Respiratory & 28 \\
\hline 318 & Osteoarthrosis, generalized, hand & 71504 & 7828 & $\mathrm{MN}$ & Musculoskeletal & 35 \\
\hline 320 & Benign neoplasm of skin of upper limb... & 2166 & 5656 & $\mathrm{MN}$ & Integumentary & 19 \\
\hline 321 & Other and unspecified disc disorder, ... & 72293 & 242473 & $\mathrm{MN}$ & Musculoskeletal & 42 \\
\hline 322 & Sinoatrial node dysfunction & 42781 & 50014 & $\mathrm{MN}$ & Cardiovascular & 66 \\
\hline 323 & Respiratory failure & 51881 & 16822 & $\mathrm{MN}$ & Respiratory & 92 \\
\hline 324 & Deviated nasal septum & 470 & 76618 & $\mathrm{MN}$ & Special.Sensory & 28 \\
\hline 325 & Hypogammaglobulinemia, unspecified & 27900 & 15980 & $\mathrm{MN}$ & Hematologic.Immune & 49 \\
\hline 328 & Contusion of shoulder region & 92300 & 167031 & $\mathrm{MN}$ & Musculoskeletal & 20 \\
\hline
\end{tabular}




\begin{tabular}{|c|c|c|c|c|c|c|}
\hline Item & ICD-9 Description & ICD-9 Code & $\begin{array}{l}\text { Weighted } \\
\text { Frequency }\end{array}$ & $\begin{array}{l}\text { Decision } \\
\text { Round }\end{array}$ & TPS Category & $\mathrm{IoH}$ \\
\hline 329 & Chronic pyelonephritis w/o lesion of ... & 59000 & 27114 & $\mathrm{EC}$ & Nephrologic & 64 \\
\hline 330 & Mallet finger & 7361 & 30920 & $\mathrm{MN}$ & Musculoskeletal & 30 \\
\hline 332 & Cough & 7862 & 3457527 & $\mathrm{MN}$ & Respiratory & 33 \\
\hline 333 & Opn wound foot except toe(s) alone w/. . . & 8920 & 47110 & $\mathrm{MN}$ & Integumentary & 37 \\
\hline 334 & Labyrinthitis, unspecified & 38630 & 124992 & $\mathrm{MN}$ & Special.Sensory & 44 \\
\hline 335 & Gastrc ulcr unspc acut/chrnc w/o men ... & 53190 & 50137 & $\mathrm{MN}$ & Gastrointestinal & 53 \\
\hline 337 & Diabetes w/ophthalmic manifestations,... & 25052 & 6652 & $\mathrm{MN}$ & Endocrine & 62 \\
\hline 338 & Obstruct chronic bronchitis w/out exacer & 49120 & 63126 & $\mathrm{MN}$ & Respiratory & 49 \\
\hline 339 & Endocarditis, valve unspecified, unsp... & 42490 & 30111 & $\mathrm{MN}$ & Cardiovascular & 83 \\
\hline 340 & Esophagitis, unspecified & 53010 & 118079 & $\mathrm{MN}$ & Gastrointestinal & 46 \\
\hline 341 & Other isolated or simple phobias & 30029 & 5234 & $\mathrm{MN}$ & Psychogenic & 42 \\
\hline 342 & Abrasion or friction burn of finger(s. . & 9150 & 41586 & $\mathrm{MN}$ & Integumentary & 18 \\
\hline 343 & Phlebitis \& thrombophlebitis of super. . & 4510 & 32888 & $\mathrm{EC}$ & Cardiovascular & 44 \\
\hline 344 & Nonallopathic lesions, sacral region & 7394 & 35769 & $\mathrm{MN}$ & Musculoskeletal & 33 \\
\hline 345 & Other specified drug dependence, unsp... & 30460 & 66059 & $\mathrm{MN}$ & Psychogenic & 53 \\
\hline 347 & Decubitus ulcer of other site & 70709 & 3591 & $\mathrm{MN}$ & Integumentary & 48 \\
\hline 348 & Spinal stenosis of cervical region & 7230 & 65423 & EC & Neurologic & 60 \\
\hline 349 & Dizziness and giddiness & 7804 & 1369179 & $\mathrm{EC}$ & Neurologic & 49 \\
\hline 350 & Edema of penis & 60783 & 29644 & $\mathrm{MN}$ & Reproductive.Male & 44 \\
\hline 351 & Other inflammatory disorders of male ... & 6084 & 3445 & $\mathrm{MN}$ & Reproductive.Male & 45 \\
\hline 352 & Phlebitis and thrombophlebitis of uns... & 4519 & 55086 & $\mathrm{EC}$ & Cardiovascular & 51 \\
\hline 353 & Abrasion or friction burn of elbow/fo... & 9130 & 73414 & $\mathrm{MN}$ & Integumentary & 18 \\
\hline 354 & Exostosis of jaw & 52681 & 17594 & $\mathrm{MN}$ & Musculoskeletal & 33 \\
\hline 355 & Calculus gallbladder w/o men of chole... & 57420 & 193942 & $\mathrm{MN}$ & Gastrointestinal & 42 \\
\hline 356 & Poisoning by caffeine & 96971 & 10449 & $\mathrm{EC}$ & Nonspecific & 58 \\
\hline 357 & Abnormal electrocardiogram [ECG] [EKG] & 79431 & 88267 & $\mathrm{MN}$ & Cardiovascular & 61 \\
\hline 358 & Acute gingivitis, plaque induced & 52300 & 7643 & $\mathrm{EC}$ & Special.Sensory & 35 \\
\hline 359 & Keloid scar & 7014 & 25584 & $\mathrm{MN}$ & Integumentary & 14 \\
\hline 360 & Unspecified disorder of liver & 5739 & 33006 & $\mathrm{MN}$ & Gastrointestinal & 45 \\
\hline 361 & Generalized and unspecified atheroscl. . . & 4409 & 74939 & $\mathrm{MN}$ & Cardiovascular & 58 \\
\hline 362 & Disorders of magnesium metabolism & 2752 & 14372 & $\mathrm{EC}$ & Nonspecific & 45 \\
\hline 363 & Traumat amputat ot fingr(s) (complet)... & 8860 & 28430 & $\mathrm{MN}$ & Musculoskeletal & 60 \\
\hline 364 & Uric acid nephrolithiasis & 27411 & 2838 & $\mathrm{MN}$ & Nephrologic & 55 \\
\hline 365 & $\begin{array}{l}\text { Personal history of methicillin resist staph } \\
\text { aureus }\end{array}$ & V1204 & 10574 & EC & Integumentary & 42 \\
\hline 367 & Unspecified disorder of autonomic ner. . & 3379 & 3298 & $\mathrm{MN}$ & Neurologic & 50 \\
\hline 368 & Other specified disorders of joint, p... & 71985 & 9344 & $\mathrm{MN}$ & Musculoskeletal & 35 \\
\hline 370 & Opn wound(s) (multiple) of unspec sit. . & 8798 & 174642 & $\mathrm{MN}$ & Integumentary & 38 \\
\hline 371 & Other specified disorders of shoulder & 72619 & 46806 & $\mathrm{MN}$ & Musculoskeletal & 38 \\
\hline 372 & Malignant neoplasm of ill-defined sit. . . & 1499 & 29838 & $\mathrm{MN}$ & Nonspecific & 80 \\
\hline 373 & Displacement of cervical intervertebr... & 7220 & 359692 & $\mathrm{MN}$ & Musculoskeletal & 67 \\
\hline 374 & Personal history of tobacco use & V1582 & 40258 & $\mathrm{EC}$ & Nonspecific & 58 \\
\hline 375 & External thrombosed hemorrhoids & 4554 & 26103 & $\mathrm{MN}$ & Gastrointestinal & 44 \\
\hline 376 & Clos fract dorsl [thoracic] vertebrae... & 8052 & 33635 & $\mathrm{MN}$ & Musculoskeletal & 58 \\
\hline 377 & Other disorders of external ear & 38089 & 13383 & $\mathrm{MN}$ & Special.Sensory & 30 \\
\hline 378 & Other cellulitis and abscess of upper... & 6823 & 83116 & $\mathrm{MN}$ & Integumentary & 46 \\
\hline 379 & Dyshidrosis & 70581 & 46183 & $\mathrm{MN}$ & Integumentary & 23 \\
\hline 380 & Glycosuria & 7915 & 12081 & $\mathrm{MN}$ & Endocrine & 55 \\
\hline 382 & Arthralgia of temporomandibular joint & 52462 & 46806 & $\mathrm{MN}$ & Musculoskeletal & 42 \\
\hline 384 & Unspecified disorders of pituitary gland & 2539 & 48202 & $\mathrm{MN}$ & Endocrine & 55 \\
\hline
\end{tabular}




\begin{tabular}{|c|c|c|c|c|c|c|}
\hline Item & ICD-9 Description & ICD-9 Code & $\begin{array}{l}\text { Weighted } \\
\text { Frequency }\end{array}$ & $\begin{array}{l}\text { Decision } \\
\text { Round }\end{array}$ & TPS Category & $\mathrm{IoH}$ \\
\hline 385 & Insect bite of hip/thigh/leg/ankle, n. . . & 9164 & 49646 & $\mathrm{MN}$ & Integumentary & 18 \\
\hline 386 & Late effect of certain complications ... & 9086 & 13439 & $\mathrm{MN}$ & Nonspecific & 43 \\
\hline 388 & Dyspareunia & 6250 & 9703 & $\mathrm{MN}$ & Reproductive.Female & 43 \\
\hline 389 & Unspecified osteomyelitis of ankle an. . . & 73027 & 9657 & $\mathrm{MN}$ & Musculoskeletal & 70 \\
\hline 390 & Chalazion & 3732 & 27114 & $\mathrm{EC}$ & Special.Sensory & 28 \\
\hline 391 & Clos fracture of unspecified, w/o men. . & 8058 & 95777 & $\mathrm{MN}$ & Musculoskeletal & 53 \\
\hline 392 & Closed fracture of multiple ribs, uns... & 80709 & 14771 & $\mathrm{MN}$ & Musculoskeletal & 51 \\
\hline 393 & Exercise counseling & V6541 & 51119 & $\mathrm{EC}$ & Nonspecific & 40 \\
\hline 394 & Stiffness of joint of hand, not elsew... & 71954 & 3177 & $\mathrm{MN}$ & Musculoskeletal & 25 \\
\hline 395 & Chronic gingivitis, plaque induced & 52310 & 16822 & EC & Special.Sensory & 27 \\
\hline 397 & Other disorders of muscle, ligament, ... & 72889 & 7691 & $\mathrm{MN}$ & Musculoskeletal & 33 \\
\hline 398 & Abnormal weight gain & 7831 & 451042 & $\mathrm{EC}$ & Nonspecific & 53 \\
\hline 400 & Other abnormal glucose & 79029 & 631335 & $\mathrm{MN}$ & Endocrine & 48 \\
\hline 402 & Prophylactic vaccinatn/inoculatn agns. . . & V058 & 70633 & $\mathrm{MN}$ & Population.based.Care & 56 \\
\hline 403 & Acute prostatitis & 6010 & 23164 & $\mathrm{MN}$ & Reproductive.Male & 50 \\
\hline 404 & Nonallopathic lesions, pelvic region & 7395 & 51760 & $\mathrm{MN}$ & Musculoskeletal & 24 \\
\hline 405 & Cognitive deficits & 4380 & 7828 & $\mathrm{MN}$ & Neurologic & 60 \\
\hline 406 & Other alteration of consciousness & 78009 & 82582 & $\mathrm{MN}$ & Neurologic & 68 \\
\hline 407 & Contraceptive pill & V2541 & 20879 & $\mathrm{MN}$ & Reproductive.Female & 54 \\
\hline 408 & Enuresis & 3076 & 28430 & $\mathrm{EC}$ & Nephrologic & 47 \\
\hline 410 & Osteoarthrosis, local, not spec prim/... & 71536 & 45577 & $M N$ & Musculoskeletal & 31 \\
\hline 411 & Obstructive sleep apnea(adult)(pediatric & 32723 & 852029 & $\mathrm{MN}$ & Respiratory & 60 \\
\hline 412 & Scabies & 1330 & 250339 & $\mathrm{MN}$ & Integumentary & 33 \\
\hline 414 & Abdominal tenderness, epigastric & 78966 & 18558 & $\mathrm{MN}$ & Gastrointestinal & 54 \\
\hline 415 & Sprains and strains of supraspinatus ... & 8406 & 10904 & $\mathrm{MN}$ & Musculoskeletal & 29 \\
\hline 416 & Unspecified diffuse connective tissue. . . & 7109 & 69171 & $M N$ & Musculoskeletal & 42 \\
\hline 418 & Duodenitis without mention of obstruc. & 53560 & 1522 & $\mathrm{MN}$ & Gastrointestinal & 48 \\
\hline 419 & Sleep disturbance, unspecified & 78050 & 373889 & $\mathrm{MN}$ & Psychogenic & 40 \\
\hline 420 & Abnormal findings in stool contents & 7921 & 14372 & $\mathrm{MN}$ & Gastrointestinal & 33 \\
\hline 421 & Disruption of external operation woun. . . & 99832 & 4498 & $\mathrm{MN}$ & Integumentary & 46 \\
\hline 422 & Contracture of joint of shoulder region & 71841 & 10449 & $\mathrm{MN}$ & Musculoskeletal & 44 \\
\hline 423 & Fitting and adjustment of urinary dev. . . & V536 & 11706 & EC & Nephrologic & 33 \\
\hline 424 & Diabetes mellitus w/o complication, t. . & 25002 & 597071 & $\mathrm{MN}$ & Endocrine & 67 \\
\hline 425 & Muscle weakness & 72887 & 138874 & EC & Musculoskeletal & 56 \\
\hline 428 & Unspecified disorder of penis & 6079 & 14334 & $\mathrm{MN}$ & Reproductive.Male & 35 \\
\hline 429 & Acute pyelonephritis without lesion o... & 59010 & 55543 & $\mathrm{MN}$ & Nephrologic & 65 \\
\hline 431 & Adjustment reaction with anxious mood & 30924 & 64936 & $M N$ & Psychogenic & 50 \\
\hline 432 & Acute tracheitis with obstruction & 46411 & 18558 & $\mathrm{MN}$ & Respiratory & 72 \\
\hline 433 & Unspecified immunity deficiency & 2793 & 8004 & $\mathrm{MN}$ & Hematologic.Immune & 56 \\
\hline 434 & Other specified disorders of female geni. . . & 62989 & 27169 & $\mathrm{MN}$ & Reproductive.Female & 46 \\
\hline 435 & Unspecified musculoskeletal disorders. . . & 7239 & 95519 & $\mathrm{MN}$ & Musculoskeletal & 29 \\
\hline 436 & Abnormal kidney function study & 7944 & 42955 & $\mathrm{MN}$ & Nephrologic & 51 \\
\hline 437 & Unspecified vascular insufficiency of. . . & 5579 & 17594 & $\mathrm{MN}$ & Cardiovascular & 53 \\
\hline 438 & Oral aphthae of the oral soft tissues & 5282 & 31214 & $\mathrm{EC}$ & Special.Sensory & 27 \\
\hline 439 & Sprains and strains of unspecified si... & 8409 & 434024 & $\mathrm{MN}$ & Musculoskeletal & 23 \\
\hline 440 & $\begin{array}{l}\text { Pap smear cerv/w atypic squamous cells of } \\
\text { undeter significance }\end{array}$ & 79501 & 37630 & $\mathrm{MN}$ & Reproductive.Female & 54 \\
\hline 441 & Enthesopathy of hip region & 7265 & 357003 & $\mathrm{MN}$ & Musculoskeletal & 33 \\
\hline 442 & Spasmodic torticollis & 33383 & 12309 & $M N$ & Musculoskeletal & 46 \\
\hline 443 & Psychogenic pain, site unspecified & 30780 & 8838 & $\mathrm{MN}$ & Psychogenic & 48 \\
\hline
\end{tabular}




\begin{tabular}{|c|c|c|c|c|c|c|}
\hline Item & ICD-9 Description & ICD-9 Code & $\begin{array}{l}\text { Weighted } \\
\text { Frequency }\end{array}$ & $\begin{array}{c}\text { Decision } \\
\text { Round }\end{array}$ & TPS Category & $\mathrm{IoH}$ \\
\hline 444 & Observation for other spec susp condit.. & V7189 & 192375 & $\mathrm{MN}$ & Nonspecific & 27 \\
\hline 445 & Cystocele, midline & 61801 & 35921 & EC & Nephrologic & 43 \\
\hline 446 & Other acquired absence of organ & V4579 & 42587 & $\mathrm{MN}$ & Nonspecific & 27 \\
\hline 447 & Degeneration of lumbar or lumbosacral. . . & 72252 & 525041 & $\mathrm{MN}$ & Musculoskeletal & 48 \\
\hline 448 & Closed fracture of sternum & 8072 & 43951 & $\mathrm{MN}$ & Musculoskeletal & 50 \\
\hline 449 & Obstruction of Eustachian tube, unspe... & 38160 & 46806 & $\mathrm{MN}$ & Special.Sensory & 35 \\
\hline 450 & Hydronephrosis & 591 & 27114 & $\mathrm{MN}$ & Nephrologic & 67 \\
\hline 451 & Lesion of plantar nerve & 3556 & 38632 & $\mathrm{MN}$ & Neurologic & 38 \\
\hline 452 & Prostatitis, unspecified & 6019 & 184973 & $\mathrm{MN}$ & Reproductive.Male & 50 \\
\hline 453 & Other acne & 7061 & 739842 & $\mathrm{MN}$ & Integumentary & 35 \\
\hline 455 & Other specified disorders of liver & 5738 & 33530 & $\mathrm{MN}$ & Gastrointestinal & 46 \\
\hline 456 & Elevated prostate specific antigen [PSA] & 79093 & 121841 & $\mathrm{MN}$ & Reproductive.Male & 50 \\
\hline 457 & Postmenopausal hormone replacement th. & V074 & 60150 & $\mathrm{MN}$ & Reproductive.Female & 44 \\
\hline 458 & Unspecified cause & 6929 & 1926987 & $\mathrm{MN}$ & Nonspecific & 17 \\
\hline 460 & Aortic valve disorders & 4241 & 125626 & $\mathrm{MN}$ & Cardiovascular & 67 \\
\hline 461 & Idiopath normal pressure hydroceph (INPH) & 3315 & 6973 & $\mathrm{MN}$ & Neurologic & 60 \\
\hline 462 & Nontoxic multinodular goiter & 2411 & 8265 & $\mathrm{MN}$ & Endocrine & 48 \\
\hline 463 & Rectal prolapse & 5691 & 24095 & $\mathrm{MN}$ & Gastrointestinal & 52 \\
\hline 464 & Sprains and strains of cruciate ligam... & 8442 & 20484 & $\mathrm{MN}$ & Musculoskeletal & 42 \\
\hline 465 & Other lymphatic and hematopoietic tissues & 23989 & 13045 & $\mathrm{MN}$ & Hematologic.Immune & 42 \\
\hline 466 & Closed dislocation of interphalangeal. . . & 83402 & 16202 & $\mathrm{MN}$ & Musculoskeletal & 48 \\
\hline 467 & Previous cesarean delivery, del w or ... & 65421 & 11942 & $\mathrm{MN}$ & Reproductive.Female & 39 \\
\hline 468 & Urinary frequency & 78841 & 509208 & $\mathrm{EC}$ & Nephrologic & 52 \\
\hline 469 & Early satiety & 78094 & 18233 & $\mathrm{MN}$ & Gastrointestinal & 46 \\
\hline 471 & Epilepsy, unspecified without mention. . . & 34590 & 384770 & $\mathrm{MN}$ & Neurologic & 69 \\
\hline 472 & Unspecified visual disturbance & 3689 & 96070 & $\mathrm{MN}$ & Special.Sensory & 58 \\
\hline 473 & Venous embolism and thrombosis of unspec & 45340 & 420827 & $\mathrm{MN}$ & Cardiovascular & 75 \\
\hline 474 & Balanoposthitis & 6071 & 27114 & $\mathrm{MN}$ & Reproductive.Male & 44 \\
\hline 477 & Secondary cardiomyopathy, unspecified & 4259 & 4014 & $\mathrm{MN}$ & Cardiovascular & 67 \\
\hline 478 & Complications of transplanted organ, .. & 99680 & 28430 & $\mathrm{MN}$ & Nonspecific & 62 \\
\hline 480 & Other specified rehabilitation procedure & V5789 & 8459 & $\mathrm{MN}$ & Nonspecific & 32 \\
\hline 481 & Acute poliomyelitis, unspecified poli. . & 4590 & 27114 & $\mathrm{EC}$ & Neurologic & 65 \\
\hline 482 & Psoriatic arthropathy & 6960 & 11643 & $\mathrm{MN}$ & Musculoskeletal & 52 \\
\hline 483 & Unspecified extrapyramidal disease an. . & 33390 & 3746 & $\mathrm{EC}$ & Neurologic & 53 \\
\hline 484 & Unspecified disorder of lipoid metabo... & 2729 & 163937 & $\mathrm{EC}$ & Cardiovascular & 50 \\
\hline 485 & Toxic effect of venom & 9895 & 101181 & $\mathrm{MN}$ & Nonspecific & 56 \\
\hline 486 & Other anomalies of larynx, trachea, a... & 7483 & 3498 & $\mathrm{EC}$ & Respiratory & 44 \\
\hline 488 & Predominant disturbance of emotions & 3080 & 18061 & $\mathrm{MN}$ & Psychogenic & 38 \\
\hline 489 & Pulmonary valve disorders & 4243 & 3897 & $\mathrm{MN}$ & Cardiovascular & 58 \\
\hline 492 & Other aneurysm of unspecified site & 4429 & 10732 & $\mathrm{MN}$ & Cardiovascular & 65 \\
\hline 493 & Unspecified cerebrovascular disease & 4379 & 46261 & $\mathrm{EC}$ & Neurologic & 65 \\
\hline 494 & Other specified retention of urine & 78829 & 12312 & $\mathrm{EC}$ & Nephrologic & 50 \\
\hline 496 & Other testicular hypofunction & 2572 & 999048 & $\mathrm{MN}$ & Reproductive.Male & 44 \\
\hline 499 & Hemangioma of unspecified site & 22800 & 27085 & $\mathrm{MN}$ & Integumentary & 24 \\
\hline 500 & Malignant neoplasm of esophagus, unsp. . & 1509 & 22518 & $\mathrm{MN}$ & Gastrointestinal & 72 \\
\hline 501 & Sprains and strains of unspecified site & 84210 & 68227 & $\mathrm{MN}$ & Musculoskeletal & 33 \\
\hline 503 & Dehydration & 27651 & 81224 & $\mathrm{EC}$ & Nonspecific & 59 \\
\hline 504 & Chronic laryngotracheitis & 4761 & 11161 & $\mathrm{MN}$ & Respiratory & 44 \\
\hline 505 & Open wound of scalp, without mention ... & 8730 & 53708 & $\mathrm{MN}$ & Integumentary & 35 \\
\hline
\end{tabular}




\begin{tabular}{|c|c|c|c|c|c|c|}
\hline Item & ICD-9 Description & ICD-9 Code & $\begin{array}{l}\text { Weighted } \\
\text { Frequency }\end{array}$ & $\begin{array}{l}\text { Decision } \\
\text { Round }\end{array}$ & TPS Category & $\mathrm{IoH}$ \\
\hline 506 & Insect bite of hip, thigh, leg, and a. . & 9165 & 14888 & $\mathrm{MN}$ & Integumentary & 24 \\
\hline 507 & Unspecified adverse effect of unspecifie... & 99520 & 91428 & $\mathrm{MN}$ & Nonspecific & 33 \\
\hline 508 & Other and unspecified sleep apnea & 78057 & 710888 & $\mathrm{MN}$ & Respiratory & 56 \\
\hline 509 & Other unknown and unspecified cause o... & 7999 & 22753 & $\mathrm{MN}$ & Nonspecific & 29 \\
\hline 511 & Anal fissure & 5650 & 27616 & $\mathrm{MN}$ & Gastrointestinal & 39 \\
\hline 512 & Viral labyrinthitis & 38635 & 40088 & $\mathrm{MN}$ & Special.Sensory & 40 \\
\hline 513 & Cardiovascular disease, unspecified & 4292 & 15955 & $\mathrm{MN}$ & Cardiovascular & 52 \\
\hline 514 & Malignant neoplasm of uterus, part un. . & 179 & 27114 & $\mathrm{MN}$ & Reproductive.Female & 72 \\
\hline 515 & Personal history of other malignant $\mathrm{n} .$. & V1083 & 37630 & $\mathrm{EC}$ & Integumentary & 60 \\
\hline 516 & Hyperplasia of prostate, unsp w/o uri. . . & 60090 & 28811 & $\mathrm{MN}$ & Reproductive.Male & 37 \\
\hline 517 & Abnormal coagulation profile & 79092 & 17594 & $\mathrm{MN}$ & Hematologic.Immune & 56 \\
\hline 518 & Oth spec infec/parasit dis, antepartu... & 64783 & 5008 & $\mathrm{MN}$ & Reproductive.Female & 54 \\
\hline 519 & Fussy infant (baby) & 78091 & 8449 & $\mathrm{EC}$ & Nonspecific & 37 \\
\hline 520 & Pain in joint of forearm & 71943 & 288591 & $\mathrm{MN}$ & Musculoskeletal & 35 \\
\hline 521 & Chills (without fever) & 78064 & 70662 & $\mathrm{MN}$ & Nonspecific & 41 \\
\hline 522 & Malignant neoplasm of rectum & 1541 & 2491 & $\mathrm{MN}$ & Gastrointestinal & 79 \\
\hline 523 & Knee, leg, ankle, and foot injury, ot. . . & 9597 & 131066 & $\mathrm{MN}$ & Musculoskeletal & 40 \\
\hline 524 & Cramp & 72982 & 228802 & $\mathrm{MN}$ & Musculoskeletal & 27 \\
\hline 525 & Pes anserinus tendinitis or bursitis & 72661 & 5159 & $\mathrm{MN}$ & Musculoskeletal & 40 \\
\hline 526 & Intermediate coronary syndrome & 4111 & 1522 & $\mathrm{MN}$ & Cardiovascular & 69 \\
\hline 529 & Clos skl frc w/o mention intracrn inj... & 80300 & 37630 & $\mathrm{EC}$ & Musculoskeletal & 56 \\
\hline 530 & Other cellulitis and abscess of trunk & 6822 & 140047 & $\mathrm{MN}$ & Integumentary & 45 \\
\hline 531 & Nonallopathic lesions, rib cage & 7398 & 10432 & $\mathrm{MN}$ & Musculoskeletal & 36 \\
\hline 532 & Osteoarthrosis, generalized, multiple... & 71509 & 81405 & $\mathrm{MN}$ & Musculoskeletal & 45 \\
\hline 533 & $\begin{array}{l}\text { Personal history oth genital sys and } \\
\text { obstetric di... }\end{array}$ & V1329 & 27114 & $\mathrm{MN}$ & Reproductive.Female & 29 \\
\hline 534 & Prophylactic vaccination/inoculation ... & V045 & 46806 & $\mathrm{MN}$ & Population.based.Care & 56 \\
\hline 535 & Oth abnor findgs on radio exam breast... & 79389 & 53367 & $\mathrm{EC}$ & Reproductive.Female & 62 \\
\hline 536 & Spondylosis of unspecified site witho... & 72190 & 49194 & $\mathrm{MN}$ & Musculoskeletal & 34 \\
\hline 537 & Other acute postoperative pain & 33818 & 75630 & $\mathrm{MN}$ & Nonspecific & 45 \\
\hline 538 & Diverticulitis of colon (without ment. . . & 56211 & 370521 & $\mathrm{MN}$ & Gastrointestinal & 69 \\
\hline 539 & Bipolar affective disorder, depressed... & 29650 & 48712 & $\mathrm{MN}$ & Psychogenic & 63 \\
\hline 540 & Blister of trunk without mention of i. . & 9112 & 1830 & $\mathrm{MN}$ & Integumentary & 18 \\
\hline 541 & Nonallopathic lesions, lower extremities & 7396 & 12309 & $\mathrm{MN}$ & Musculoskeletal & 33 \\
\hline 543 & Sprains and strains of unspecified si... & 8479 & 174720 & $\mathrm{MN}$ & Musculoskeletal & 30 \\
\hline 544 & Amblyopia, unspecified & 36800 & 3453 & $\mathrm{MN}$ & Special.Sensory & 47 \\
\hline 545 & Hypertonicity of bladder & 59651 & 154116 & $\mathrm{EC}$ & Nephrologic & 43 \\
\hline 546 & Other specified disorders of thyroid & 2468 & 11533 & $\mathrm{MN}$ & Endocrine & 51 \\
\hline 548 & Pain in joint of ankle and foot & 71947 & 659552 & $\mathrm{MN}$ & Musculoskeletal & 34 \\
\hline 549 & Vascular disorders of penis & 60782 & 27114 & $\mathrm{MN}$ & Reproductive.Male & 45 \\
\hline 551 & Obstructive hydrocephalus & 3314 & 9554 & $\mathrm{MN}$ & Neurologic & 78 \\
\hline 552 & Degeneration of intervertebral disc, ... & 7226 & 595663 & $\mathrm{MN}$ & Musculoskeletal & 53 \\
\hline 553 & Osteoarthrosis, unspec generalized or... & 71596 & 487109 & $\mathrm{MN}$ & Musculoskeletal & 45 \\
\hline 554 & Tietze`s disease & 7336 & 215211 & $\mathrm{MN}$ & Musculoskeletal & 31 \\
\hline 555 & Other cardiac dysrhythmias & 42789 & 213963 & $\mathrm{MN}$ & Cardiovascular & 66 \\
\hline 556 & Heart valve replacement & V433 & 21475 & $\mathrm{MN}$ & Cardiovascular & 58 \\
\hline 557 & Conjunctivitis, unspecified & 37230 & 482456 & $\mathrm{MN}$ & Special.Sensory & 33 \\
\hline 558 & Malignant neoplasm of liver/not speci. . . & 1552 & 12176 & $\mathrm{MN}$ & Gastrointestinal & 69 \\
\hline 559 & Talipes, unspecified & 75470 & 17594 & $\mathrm{MN}$ & Musculoskeletal & 46 \\
\hline
\end{tabular}




\begin{tabular}{|c|c|c|c|c|c|c|}
\hline Item & ICD-9 Description & ICD-9 Code & $\begin{array}{l}\text { Weighted } \\
\text { Frequency }\end{array}$ & $\begin{array}{l}\text { Decision } \\
\text { Round }\end{array}$ & TPS Category & $\mathrm{IoH}$ \\
\hline 560 & Universal ulcerative (chronic) colitis & 5566 & 12647 & $\mathrm{MN}$ & Gastrointestinal & 71 \\
\hline 561 & Dermatophytosis of foot & 1104 & 187862 & $\mathrm{MN}$ & Integumentary & 21 \\
\hline 562 & Lichenification and lichen simplex ch... & 6983 & 73686 & $\mathrm{MN}$ & Integumentary & 28 \\
\hline 563 & Pulmonary tuberculosis, unspecified & 1190 & 13006 & $\mathrm{MN}$ & Respiratory & 74 \\
\hline 564 & Viral warts, unspecified & 7810 & 466770 & $\mathrm{MN}$ & Integumentary & 19 \\
\hline 565 & Open wound of forehead & 87342 & 15899 & $\mathrm{MN}$ & Integumentary & 39 \\
\hline 566 & Arthropathy, unspecified, pelvic regi... & 71695 & 30677 & $\mathrm{MN}$ & Musculoskeletal & 41 \\
\hline 567 & Disuse osteoporosis & 73303 & 5234 & $\mathrm{MN}$ & Musculoskeletal & 47 \\
\hline 568 & Chronic migraine without aura, without.. & 34670 & 39619 & $\mathrm{MN}$ & Neurologic & 56 \\
\hline 569 & Personal history of pulmonary embolism & $\mathrm{V} 1255$ & 48534 & EC & Cardiovascular & 62 \\
\hline 570 & Candidiasis of mouth & 1120 & 168454 & $\mathrm{EC}$ & Special.Sensory & 24 \\
\hline 571 & Attention deficit disorder with hyper... & 31401 & 1320982 & $\mathrm{MN}$ & Psychogenic & 51 \\
\hline 572 & Secondary diabetes mellitus w/o comp... & 24900 & 5656 & $\mathrm{MN}$ & Endocrine & 66 \\
\hline 573 & Mononeuritis of upper limb & 3549 & 46220 & $\mathrm{MN}$ & Neurologic & 49 \\
\hline 574 & Calcaneal spur & 72673 & 50741 & $\mathrm{MN}$ & Musculoskeletal & 23 \\
\hline 575 & Arthropathy, unspecified, ankle and foot & 71697 & 11942 & $\mathrm{MN}$ & Musculoskeletal & 30 \\
\hline 576 & Chronic interstitial cystitis & 5951 & 5656 & $\mathrm{EC}$ & Nephrologic & 47 \\
\hline 577 & Osteodystrophy, unspecified & 75650 & 46806 & $\mathrm{MN}$ & Musculoskeletal & 37 \\
\hline 578 & Acute pharyngitis & 462 & 3546180 & $\mathrm{EC}$ & Respiratory & 38 \\
\hline 580 & Opioid abuse, unspecified & 30550 & 151346 & $\mathrm{MN}$ & Psychogenic & 60 \\
\hline 581 & Chronic fatigue syndrome & 78071 & 77434 & $\mathrm{EC}$ & Nonspecific & 47 \\
\hline 582 & Hemorrhage of rectum and anus & 5693 & 213065 & $\mathrm{MN}$ & Gastrointestinal & 66 \\
\hline 583 & Degeneration of cervical intervertebr... & 7224 & 184179 & $\mathrm{MN}$ & Musculoskeletal & 47 \\
\hline 584 & Unspecified dermatitis due to sun & 69270 & 4533 & $\mathrm{MN}$ & Integumentary & 26 \\
\hline 585 & Personal history of urinary calculi & V1301 & 29681 & $\mathrm{EC}$ & Nephrologic & 39 \\
\hline 586 & Alcohol abuse, episodic & 30502 & 21003 & $\mathrm{EC}$ & Psychogenic & 60 \\
\hline 587 & Chronic obstructive asthma without me. . & 49320 & 172201 & $\mathrm{MN}$ & Respiratory & 60 \\
\hline 588 & Acute myocard infarc of oth anter wal. . & 41011 & 1522 & $\mathrm{MN}$ & Cardiovascular & 93 \\
\hline 589 & Unspecified disorder of joint, lower leg & 71996 & 7445 & $\mathrm{MN}$ & Musculoskeletal & 30 \\
\hline 590 & Altered mental status & 78097 & 47340 & $\mathrm{MN}$ & Neurologic & 78 \\
\hline 591 & Unspecified degree burn of face and h... & 94100 & 17572 & $\mathrm{MN}$ & Integumentary & 58 \\
\hline 592 & Special screening for nephropathy & V815 & 32834 & $\mathrm{MN}$ & Nephrologic & 39 \\
\hline 593 & Closed fracture of unspecified part o... & 82381 & 17421 & $\mathrm{MN}$ & Musculoskeletal & 48 \\
\hline 595 & Meralgia paresthetica & 3551 & 16622 & $\mathrm{MN}$ & Neurologic & 39 \\
\hline 596 & Other symptoms involving skin and int... & 7829 & 58488 & $\mathrm{MN}$ & Integumentary & 24 \\
\hline 597 & Carbuncle and furuncle of face & 6800 & 9009 & $\mathrm{MN}$ & Integumentary & 37 \\
\hline 598 & Open wound of forearm, without mentio... & 88100 & 39688 & $\mathrm{MN}$ & Integumentary & 39 \\
\hline 599 & Other specified disorders of nervous ... & 34989 & 17380 & $\mathrm{MN}$ & Neurologic & 47 \\
\hline 600 & Nonspecific abnormal toxicological fi... & 7960 & 54741 & $\mathrm{MN}$ & Nonspecific & 42 \\
\hline 601 & Personal history of colonic polyps & V1272 & 48636 & $\mathrm{MN}$ & Gastrointestinal & 50 \\
\hline 602 & Unspecified disorder of thyroid & 2469 & 344713 & $\mathrm{MN}$ & Endocrine & 50 \\
\hline 604 & Pilonidal cyst without mention of abs. . . & 6851 & 62962 & $\mathrm{MN}$ & Integumentary & 38 \\
\hline 605 & Thyrotoxicosis with toxic diffuse goi... & 24200 & 55543 & $\mathrm{MN}$ & Endocrine & 76 \\
\hline 606 & Bacterial pneumonia unspecified & 4829 & 105746 & $\mathrm{MN}$ & Respiratory & 76 \\
\hline 607 & Paranoid type of schizophrenic disord. . & 29530 & 6911 & $\mathrm{MN}$ & Psychogenic & 65 \\
\hline 608 & Proteinuria & 7910 & 91740 & $\mathrm{MN}$ & Nephrologic & 52 \\
\hline 609 & Unspecified genital prolapse & 6189 & 15464 & $\mathrm{MN}$ & Reproductive.Female & 46 \\
\hline 610 & Factitious illness with psychological. . & 30016 & 12477 & $\mathrm{MN}$ & Psychogenic & 42 \\
\hline 611 & Calculus of kidney & 5920 & 364745 & $\mathrm{MN}$ & Nephrologic & 57 \\
\hline 612 & Other disorders of bone and cartilage & 73399 & 16639 & $\mathrm{MN}$ & Musculoskeletal & 40 \\
\hline
\end{tabular}




\begin{tabular}{|c|c|c|c|c|c|c|}
\hline Item & ICD-9 Description & ICD-9 Code & $\begin{array}{l}\text { Weighted } \\
\text { Frequency }\end{array}$ & $\begin{array}{l}\text { Decision } \\
\text { Round }\end{array}$ & TPS Category & $\mathrm{IoH}$ \\
\hline 613 & Chondromalacia of patella & 7177 & 31707 & $\mathrm{MN}$ & Musculoskeletal & 34 \\
\hline 614 & Abscess of anal and rectal regions & 566 & 3746 & $\mathrm{MN}$ & Gastrointestinal & 61 \\
\hline 615 & Other specified disorders of rectum a... & 56949 & 37648 & $\mathrm{MN}$ & Gastrointestinal & 42 \\
\hline 616 & Unspecified hemorrhoids without menti. . . & 4556 & 219386 & $\mathrm{MN}$ & Gastrointestinal & 38 \\
\hline 617 & Other symptoms referable to back & 7248 & 218010 & $\mathrm{MN}$ & Musculoskeletal & 34 \\
\hline 618 & Neurotic depression & 3004 & 754576 & $\mathrm{MN}$ & Psychogenic & 52 \\
\hline 619 & Sprains and strains of unspecified si... & 8439 & 22259 & $\mathrm{MN}$ & Musculoskeletal & 29 \\
\hline 620 & Methicillin resistant Staphylococcus aureus & 4112 & 87685 & $\mathrm{EC}$ & Integumentary & 65 \\
\hline 621 & Infectious mononucleosis & 75 & 17824 & $\mathrm{EC}$ & Hematologic.Immune & 48 \\
\hline 622 & Other peripheral vertigo & 38619 & 14219 & $\mathrm{EC}$ & Neurologic & 44 \\
\hline 623 & Other high-risk pregnancy & V2389 & 342236 & $\mathrm{MN}$ & Reproductive.Female & 65 \\
\hline 624 & Special screening for hypertension & V811 & 128680 & $\mathrm{MN}$ & Cardiovascular & 52 \\
\hline 625 & Other spec early childhood psychoses,... & 29980 & 8103 & $\mathrm{MN}$ & Psychogenic & 61 \\
\hline 626 & Phlebitis \& thrombophlebitis of oth d... & 45119 & 33104 & $\mathrm{MN}$ & Cardiovascular & 50 \\
\hline 628 & Unspecified open wound of eyeball & 8719 & 24878 & $\mathrm{MN}$ & Special.Sensory & 72 \\
\hline 629 & Closed fracture of ulna (alone) & 81382 & 16617 & $\mathrm{MN}$ & Musculoskeletal & 54 \\
\hline 630 & Lesion of ulnar nerve & 3542 & 36121 & $\mathrm{MN}$ & Neurologic & 54 \\
\hline 631 & Hypertrophy (benign) prostate w/o uri. . . & 60000 & 516532 & $\mathrm{MN}$ & Reproductive.Male & 44 \\
\hline 632 & Neoplasm of uncertain behavior of testis & 2364 & 3798 & $\mathrm{MN}$ & Reproductive.Male & 72 \\
\hline 633 & Poisoning by other & 96509 & 5697 & $\mathrm{MN}$ & Nonspecific & 70 \\
\hline 635 & Diabetes w/neurological manifestation. . . & 25062 & 101985 & $\mathrm{MN}$ & Endocrine & 65 \\
\hline 636 & Closed fracture of radius (alone) & 81381 & 57919 & $\mathrm{MN}$ & Musculoskeletal & 54 \\
\hline 637 & Coronary atherosclerosis of native co... & 41401 & 195986 & $\mathrm{MN}$ & Cardiovascular & 76 \\
\hline 638 & Pyelonephritis, unspecified & 59080 & 102794 & $\mathrm{MN}$ & Nephrologic & 70 \\
\hline 640 & Abrasion or friction burn of hip/thig. . & 9161 & 8936 & $\mathrm{MN}$ & Integumentary & 17 \\
\hline 641 & Insect bite of elbow/forearm/wrist, n. . . & 9134 & 29644 & $\mathrm{MN}$ & Integumentary & 17 \\
\hline 642 & Acute tonsillitis & 463 & 369522 & $\mathrm{EC}$ & Respiratory & 42 \\
\hline 644 & Presbyacusis & 38801 & 4738 & $\mathrm{MN}$ & Special.Sensory & 42 \\
\hline 645 & Arthropathy, unspecified, shoulder re... & 71691 & 5798 & $\mathrm{MN}$ & Musculoskeletal & 34 \\
\hline 646 & Conjunctival hemorrhage & 37272 & 31876 & $\mathrm{MN}$ & Special.Sensory & 29 \\
\hline 647 & Abnormal mammogram, unspecified & 79380 & 18849 & $\mathrm{EC}$ & Reproductive.Female & 59 \\
\hline 648 & Redundant prepuce and phimosis & 605 & 7136 & $\mathrm{MN}$ & Reproductive.Male & 40 \\
\hline 649 & Urticaria, unspecified & 7089 & 236512 & EC & Integumentary & 48 \\
\hline 650 & Nonspec elevatn of transaminase or la... & 7904 & 108010 & $\mathrm{MN}$ & Gastrointestinal & 44 \\
\hline 652 & Systolic heart failure, unspecified & 42820 & 52634 & $\mathrm{MN}$ & Cardiovascular & 72 \\
\hline 653 & Chronic kidney disease, stage IV(severe) & 5854 & 8698 & $\mathrm{MN}$ & Nephrologic & 79 \\
\hline 654 & Other specified conditions influencin... & V4989 & 28430 & $\mathrm{MN}$ & Nonspecific & 29 \\
\hline 655 & Other symptoms referable to joint of ... & 71962 & 1766 & $\mathrm{MN}$ & Musculoskeletal & 25 \\
\hline 656 & Renal dialysis status & V4511 & 28430 & $\mathrm{MN}$ & Nephrologic & 72 \\
\hline 657 & Other specified forms of chronic isch... & 4148 & 48261 & $\mathrm{MN}$ & Cardiovascular & 60 \\
\hline 658 & Bipolar affective disorder, mixed, un. .. & 29660 & 10334 & $\mathrm{MN}$ & Psychogenic & 55 \\
\hline 660 & Major depressive disorder, single epi. . . & 29626 & 17148 & $\mathrm{MN}$ & Psychogenic & 61 \\
\hline 662 & Special screening for lipoid disorders & V7791 & 62054 & $\mathrm{EC}$ & Cardiovascular & 38 \\
\hline 663 & Unspecified degree burn of single dig. . . & 94401 & 28299 & $\mathrm{MN}$ & Integumentary & 32 \\
\hline 664 & Edema of male genital organs & 60886 & 46806 & $\mathrm{MN}$ & Reproductive.Male & 40 \\
\hline 665 & Periapical abscess without sinus & 5225 & 127660 & $\mathrm{EC}$ & Special.Sensory & 51 \\
\hline 666 & Sprains and strains of unspecified site & 84840 & 11398 & $\mathrm{MN}$ & Musculoskeletal & 27 \\
\hline 667 & Specl screeng for oth/unspc endocr/nu. . . & V7799 & 70272 & $\mathrm{MN}$ & Endocrine & 27 \\
\hline 668 & $\begin{array}{l}\text { Personal history other diseases circulatory } \\
\text { systm }\end{array}$ & V1259 & 132275 & $\mathrm{EC}$ & Cardiovascular & 44 \\
\hline
\end{tabular}




\begin{tabular}{|c|c|c|c|c|c|c|}
\hline Item & ICD-9 Description & ICD-9 Code & $\begin{array}{l}\text { Weighted } \\
\text { Frequency }\end{array}$ & $\begin{array}{c}\text { Decision } \\
\text { Round }\end{array}$ & TPS Category & $\mathrm{IoH}$ \\
\hline 669 & Disorders of psychosexual identity & 3026 & 16399 & $\mathrm{MN}$ & Psychogenic & 44 \\
\hline 670 & Suicidal ideation & V6284 & 4802 & $\mathrm{MN}$ & Psychogenic & 85 \\
\hline 671 & Absence of menstruation & 6260 & 149292 & $\mathrm{MN}$ & Reproductive.Female & 48 \\
\hline 672 & Acute pancreatitis & 5770 & 195740 & $\mathrm{MN}$ & Gastrointestinal & 72 \\
\hline 673 & Malignant neoplasm of colon, unspecified & 1539 & 51436 & $\mathrm{MN}$ & Gastrointestinal & 94 \\
\hline 674 & Other acute otitis externa & 38022 & 9554 & $\mathrm{MN}$ & Special.Sensory & 36 \\
\hline 675 & Other specified diseases of blood and. . & 28989 & 52405 & $\mathrm{MN}$ & Hematologic.Immune & 48 \\
\hline 676 & Localized superficial swelling, mass,... & 7822 & 209811 & $\mathrm{EC}$ & Integumentary & 34 \\
\hline 677 & Other complicated headache syndrome & 33944 & 9639 & $\mathrm{MN}$ & Neurologic & 54 \\
\hline 678 & Varicos vein of lowr extremities w/ou. . & 4549 & 133786 & $\mathrm{MN}$ & Cardiovascular & 27 \\
\hline 679 & Thyroid dysfunction, antepartum condi... & 64813 & 37666 & $\mathrm{MN}$ & Endocrine & 52 \\
\hline 680 & Mittelschmerz & 6252 & 3535 & $\mathrm{MN}$ & Reproductive.Female & 32 \\
\hline 683 & Debility, unspecified & 7993 & 58074 & $\mathrm{MN}$ & Nonspecific & 41 \\
\hline 684 & Thyroiditis, unspecified & 2459 & 50481 & $\mathrm{MN}$ & Endocrine & 54 \\
\hline 685 & Unspecified personality disorder & 3019 & 14577 & $\mathrm{MN}$ & Psychogenic & 48 \\
\hline 687 & Macular degeneration (senile), unspec. . & 36250 & 11161 & $\mathrm{MN}$ & Special.Sensory & 50 \\
\hline 688 & Cls frc cervicl vertebra, w/o ment sp... & 80500 & 37630 & $\mathrm{MN}$ & Musculoskeletal & 70 \\
\hline 689 & Unspecified sinusitis (chronic) & 4739 & 3613552 & $\mathrm{EC}$ & Respiratory & 40 \\
\hline 690 & Mild mental retardation & 317 & 2875 & $\mathrm{MN}$ & Neurologic & 44 \\
\hline 691 & Diabetes mellitus w/o complication, t. . & 25000 & 11939976 & $\mathrm{MN}$ & Endocrine & 65 \\
\hline 692 & Sprains and strains of unspecified si... & 8419 & 53133 & $\mathrm{MN}$ & Musculoskeletal & 27 \\
\hline 693 & Gross hematuria & 59971 & 5494 & $\mathrm{EC}$ & Nephrologic & 67 \\
\hline 694 & Juvenile osteochondrosis of foot & 7325 & 43428 & $\mathrm{MN}$ & Musculoskeletal & 38 \\
\hline 695 & Orbital edema or congestion & 37633 & 43115 & $\mathrm{MN}$ & Special.Sensory & 50 \\
\hline 696 & Sprains and strains of other spec sit... & 8408 & 94200 & $\mathrm{MN}$ & Musculoskeletal & 32 \\
\hline 697 & Abnormal reflex & 7961 & 14199 & $\mathrm{MN}$ & Neurologic & 44 \\
\hline 698 & Apnea & 78603 & 20804 & $\mathrm{MN}$ & Respiratory & 67 \\
\hline 699 & Abnormal thyroid function study & 7945 & 39963 & $\mathrm{MN}$ & Endocrine & 50 \\
\hline 700 & Blepharoconjunctivitis, unspecified & 37220 & 17594 & $\mathrm{MN}$ & Special.Sensory & 36 \\
\hline 702 & Prophylactic vaccinatn/inoculatn agns. . & V0382 & 38087 & $\mathrm{MN}$ & Population.based.Care & 53 \\
\hline 703 & Encounter for change or removal of surgi... & V5831 & 15749 & $\mathrm{MN}$ & Nonspecific & 28 \\
\hline 704 & Dissection of coronary artery & 41412 & 5889 & $\mathrm{MN}$ & Cardiovascular & 94 \\
\hline 705 & Other diseases of pharynx, not elsewh... & 47829 & 29838 & $\mathrm{EC}$ & Respiratory & 38 \\
\hline 706 & Other syndromes affecting cervical re... & 7238 & 45204 & $\mathrm{MN}$ & Musculoskeletal & 36 \\
\hline 707 & Senile dementia, uncomplicated & 2900 & 94175 & $\mathrm{MN}$ & Neurologic & 59 \\
\hline 708 & Herpetic whitlow & 546 & 14219 & $\mathrm{MN}$ & Integumentary & 38 \\
\hline 709 & Spasm of muscle & 72885 & 906368 & $\mathrm{MN}$ & Musculoskeletal & 24 \\
\hline 710 & Pneumococcal pneumonia [Streptococcus... & 481 & 12770 & $\mathrm{MN}$ & Respiratory & 74 \\
\hline 711 & Thoracic or lumbosacral neuritis or $\mathrm{r} . \ldots$ & 7244 & 861707 & $\mathrm{MN}$ & Neurologic & 51 \\
\hline 712 & Benign neoplasm of pituitary gland/cr. . & 2273 & 5159 & $\mathrm{MN}$ & Endocrine & 48 \\
\hline 713 & Iatrogenic pituitary disorders & 2537 & 7818 & $\mathrm{MN}$ & Endocrine & 51 \\
\hline 714 & Cytomegaloviral disease & 785 & 9344 & $\mathrm{EC}$ & Nonspecific & 49 \\
\hline 715 & Dermatophytosis of scalp and beard & 1100 & 20697 & $\mathrm{MN}$ & Integumentary & 28 \\
\hline 716 & Major depressive disorder, single epi... & 29620 & 479762 & $\mathrm{MN}$ & Psychogenic & 57 \\
\hline 717 & Alcoholic fatty liver & 5710 & 3466 & $\mathrm{MN}$ & Gastrointestinal & 51 \\
\hline 718 & Inflamed seborrheic keratosis & 70211 & 50564 & $\mathrm{MN}$ & Integumentary & 26 \\
\hline 719 & Adult failure to thrive & 7837 & 91295 & $\mathrm{MN}$ & Nonspecific & 67 \\
\hline 720 & Malignant neoplasm of pharynx, unspec. & 1490 & 14643 & $\mathrm{EC}$ & Respiratory & 71 \\
\hline 721 & Dermatomycosis, unspecified & 1119 & 56859 & $\mathrm{MN}$ & Integumentary & 26 \\
\hline
\end{tabular}




\begin{tabular}{|c|c|c|c|c|c|c|}
\hline Item & ICD-9 Description & ICD-9 Code & $\begin{array}{l}\text { Weighted } \\
\text { Frequency }\end{array}$ & $\begin{array}{l}\text { Decision } \\
\text { Round }\end{array}$ & TPS Category & $\mathrm{IoH}$ \\
\hline 722 & Tinnitus, unspecified & 38830 & 64334 & $\mathrm{MN}$ & Special.Sensory & 36 \\
\hline 723 & Impairment level not further specified & 36900 & 17492 & $\mathrm{MN}$ & Nonspecific & 34 \\
\hline 724 & Acute dermatitis due to solar radiation & 69272 & 20608 & $\mathrm{MN}$ & Integumentary & 26 \\
\hline 725 & Routine general medical examination a. . . & V700 & 9944857 & $\mathrm{EC}$ & Patient.based.Systems & 49 \\
\hline 726 & Monoclonal paraproteinemia & 2731 & 3798 & $\mathrm{MN}$ & Hematologic.Immune & 49 \\
\hline 727 & Diabetes w/neurological manifestation. .. & 25060 & 431742 & $\mathrm{MN}$ & Endocrine & 63 \\
\hline 728 & Personal history of other dis respirat system & V1269 & 86164 & $\mathrm{MN}$ & Respiratory & 42 \\
\hline 729 & Painful respiration & 78652 & 127218 & $\mathrm{MN}$ & Respiratory & 51 \\
\hline 730 & $\begin{array}{l}\text { Pap smear cerv w/low grade squamous } \\
\text { intraepithelial lesion }\end{array}$ & 79503 & 6501 & $\mathrm{MN}$ & Reproductive.Female & 55 \\
\hline 731 & Pep ulcr unsp act/chrn w/o men hem/pe... & 53390 & 128600 & $\mathrm{MN}$ & Gastrointestinal & 55 \\
\hline 732 & Bullous myringitis & 38401 & 16002 & $\mathrm{MN}$ & Special.Sensory & 46 \\
\hline 733 & Abdominal pain, other specified site & 78909 & 309123 & $\mathrm{MN}$ & Gastrointestinal & 48 \\
\hline 734 & Elevated blood pressure reading w/o d. . & 7962 & 1179330 & $\mathrm{MN}$ & Cardiovascular & 49 \\
\hline 735 & Pleurisy w/effusion, w/mention of a b... & 5111 & 3824 & $\mathrm{MN}$ & Respiratory & 61 \\
\hline 736 & Gouty arthropathy, unspecified & 27400 & 68396 & $\mathrm{MN}$ & Musculoskeletal & 49 \\
\hline 737 & Open wound of finger(s), without ment. . & 8830 & 176306 & $\mathrm{EC}$ & Integumentary & 38 \\
\hline 738 & Neoplasm of uncertain behavior of pol. . . & 2384 & 46543 & $\mathrm{EC}$ & Hematologic.Immune & 65 \\
\hline 739 & Nonallopathic lesions, lumbar region & 7393 & 264795 & $\mathrm{MN}$ & Musculoskeletal & 36 \\
\hline 740 & Calcifying tendinitis of shoulder & 72611 & 52409 & $\mathrm{MN}$ & Musculoskeletal & 38 \\
\hline 742 & Otalgia, unspecified & 38870 & 842767 & $\mathrm{MN}$ & Special.Sensory & 28 \\
\hline 743 & Unspec complic of pregnancy antepartu. . & 64693 & 3406 & $\mathrm{MN}$ & Reproductive.Female & 53 \\
\hline 744 & Jaundice, unspecified, not of newborn & 7824 & 45649 & $\mathrm{MN}$ & Gastrointestinal & 59 \\
\hline 745 & Pediculus capitis [head louse] & 1320 & 32871 & $\mathrm{MN}$ & Integumentary & 28 \\
\hline 746 & Insect bite of oth/multipl/unspec sit. . & 9194 & 245935 & $\mathrm{MN}$ & Integumentary & 19 \\
\hline 747 & Other joint derangement of shoulder $r .$. & 71881 & 1807 & $\mathrm{MN}$ & Musculoskeletal & 34 \\
\hline 748 & Bicipital tenosynovitis & 72612 & 61441 & $\mathrm{MN}$ & Musculoskeletal & 36 \\
\hline 749 & Amyotrophic lateral sclerosis & 33520 & 55010 & $\mathrm{MN}$ & Neurologic & 71 \\
\hline 750 & Prostatitis in diseases classified el. . & 6014 & 17572 & $\mathrm{MN}$ & Reproductive.Male & 50 \\
\hline 751 & Anal spasm & 5646 & 9554 & $\mathrm{MN}$ & Gastrointestinal & 26 \\
\hline 752 & $\begin{array}{l}\text { Personal history of other endocrine, } \\
\text { metab. . }\end{array}$ & V1229 & 28091 & $\mathrm{MN}$ & Endocrine & 32 \\
\hline 754 & Gastroparesis & 5363 & 38476 & $\mathrm{MN}$ & Gastrointestinal & 48 \\
\hline 755 & Disturbance of salivary secretion & 5277 & 58632 & EC & Special.Sensory & 38 \\
\hline 756 & Wheezing & 78607 & 290421 & $\mathrm{MN}$ & Respiratory & 57 \\
\hline 757 & Cirrhosis of liver without mention of. . & 5715 & 83816 & $\mathrm{MN}$ & Gastrointestinal & 67 \\
\hline 758 & Bariatric surgery status complic preg.... & 64923 & 3498 & $\mathrm{EC}$ & Reproductive.Female & 53 \\
\hline 759 & Other and unspecified parts, without... & 8748 & 15474 & $\mathrm{EC}$ & Integumentary & 26 \\
\hline 760 & Microscopic hematuria & 59972 & 66237 & $\mathrm{MN}$ & Nephrologic & 51 \\
\hline 761 & Spotting complicating pregnancy, childbir.. & 64953 & 14917 & $\mathrm{MN}$ & Reproductive.Female & 53 \\
\hline 762 & Lymphadenitis, unspecified, except me... & 2893 & 89252 & $\mathrm{EC}$ & Hematologic.Immune & 42 \\
\hline 763 & Other cellulitis and abscess of other... & 6828 & 7437 & $\mathrm{MN}$ & Integumentary & 51 \\
\hline 765 & Hemoperitoneum (nontraumatic) & 56881 & 5268 & $\mathrm{EC}$ & Gastrointestinal & 65 \\
\hline 766 & Boutonniere deformity of finger & 73621 & 2491 & $\mathrm{MN}$ & Musculoskeletal & 32 \\
\hline 767 & Pleurisy without mention of effusion ... & 5110 & 3453 & $\mathrm{MN}$ & Respiratory & 48 \\
\hline 768 & Bunion & 7271 & 52830 & $\mathrm{MN}$ & Musculoskeletal & 22 \\
\hline 769 & Other urinary incontinence & 78839 & 53015 & $\mathrm{EC}$ & Nephrologic & 46 \\
\hline 770 & Pain in joint of lower leg & 71946 & 2084138 & $\mathrm{MN}$ & Musculoskeletal & 34 \\
\hline 771 & Sprains and strains of rotator cuff $(.$. & 8404 & 137922 & $\mathrm{MN}$ & Musculoskeletal & 36 \\
\hline
\end{tabular}




\begin{tabular}{|c|c|c|c|c|c|c|}
\hline Item & ICD-9 Description & ICD-9 Code & $\begin{array}{l}\text { Weighted } \\
\text { Frequency }\end{array}$ & $\begin{array}{c}\text { Decision } \\
\text { Round }\end{array}$ & TPS Category & $\mathrm{IoH}$ \\
\hline 772 & Leukocytosis, unspecified & 28860 & 129278 & $\mathrm{MN}$ & Hematologic.Immune & 49 \\
\hline 774 & Personal history of diseases of blood. . . & V123 & 22225 & $\mathrm{MN}$ & Hematologic.Immune & 48 \\
\hline 775 & Excessive or frequent menstruation & 6262 & 277301 & $\mathrm{MN}$ & Reproductive.Female & 48 \\
\hline 776 & Other disorders of iron metabolism & 27509 & 1807 & $\mathrm{MN}$ & Hematologic.Immune & 46 \\
\hline 777 & Open wound of finger(s), complicated & 8831 & 28294 & $\mathrm{EC}$ & Integumentary & 44 \\
\hline 778 & Inflammatory disease of breast & 6110 & 77861 & $\mathrm{EC}$ & Reproductive.Female & 53 \\
\hline 779 & Other abnormality of urination & 78869 & 29644 & $\mathrm{EC}$ & Nephrologic & 32 \\
\hline 780 & Unspecified disorder of joint, should. . . & 71991 & 24421 & $\mathrm{MN}$ & Musculoskeletal & 32 \\
\hline 781 & Other conditions of brain & 34889 & 28430 & $\mathrm{MN}$ & Neurologic & 49 \\
\hline 782 & Congenital insufficiency of aortic valve & 7464 & 7691 & $\mathrm{MN}$ & Cardiovascular & 61 \\
\hline 783 & Chronic inflamm demyelinating polyneu... & 35781 & 5656 & $\mathrm{MN}$ & Neurologic & 67 \\
\hline 784 & Dermatophytosis of unspecified site & 1109 & 82877 & $\mathrm{MN}$ & Integumentary & 24 \\
\hline 785 & Streptococcal sore throat & 340 & 517440 & $\mathrm{EC}$ & Respiratory & 48 \\
\hline 786 & True post wall infarc of unspec site,... & 41090 & 15223 & $\mathrm{MN}$ & Cardiovascular & 81 \\
\hline 787 & Carbuncle and furuncle of upper arm a... & 6803 & 13209 & $\mathrm{MN}$ & Integumentary & 28 \\
\hline 788 & Chronic ulcer of other specified sites & 7078 & 4337 & $\mathrm{MN}$ & Integumentary & 38 \\
\hline 789 & Excessive crying of infant (baby) & 78092 & 21018 & $\mathrm{MN}$ & Nonspecific & 29 \\
\hline 790 & Anemia, antepartum condition or compl. . . & 64823 & 97231 & $\mathrm{EC}$ & Hematologic.Immune & 40 \\
\hline 791 & Memory loss & 78093 & 243230 & $\mathrm{MN}$ & Neurologic & 61 \\
\hline 792 & Body Mass Index 28.0 to 28.9 , adult & V8524 & 17572 & EC & Nonspecific & 30 \\
\hline 793 & Unspecified psychosis & 2989 & 132447 & $\mathrm{MN}$ & Psychogenic & 61 \\
\hline 794 & Varicose veins of lower extremities w... & 4540 & 3591 & $\mathrm{MN}$ & Cardiovascular & 30 \\
\hline 795 & Special screening for ischemic heart ... & V810 & 6022 & $\mathrm{MN}$ & Cardiovascular & 40 \\
\hline 796 & Inguinal hernia, w/o obstruc/gangrene. . . & 55090 & 167592 & $\mathrm{MN}$ & Gastrointestinal & 49 \\
\hline 797 & Hyperpotassemia & 2767 & 68700 & $\mathrm{EC}$ & Nonspecific & 65 \\
\hline 798 & Diplopia & 3682 & 32382 & $\mathrm{MN}$ & Special.Sensory & 67 \\
\hline 799 & Other psoriasis & 6961 & 342308 & $\mathrm{MN}$ & Integumentary & 44 \\
\hline 800 & Lack of normal physiological devel unsp & 78340 & 35812 & $\mathrm{EC}$ & Nonspecific & 46 \\
\hline 801 & Other insomnia & 78052 & 3069106 & $\mathrm{EC}$ & Psychogenic & 41 \\
\hline 802 & Diabetes with other coma, type II, un. . . & 25032 & 14875 & $\mathrm{MN}$ & Endocrine & 96 \\
\hline 803 & Fever and oth physio disturb of temp regu & 78060 & 709681 & $\mathrm{MN}$ & Nonspecific & 48 \\
\hline 804 & Primary exertional headache & 33984 & 1830 & $\mathrm{MN}$ & Neurologic & 48 \\
\hline 805 & Asthma, unspecified without mention o. . & 49390 & 3049441 & $\mathrm{MN}$ & Respiratory & 60 \\
\hline 806 & Suppurative labyrinthitis & 38633 & 27114 & $\mathrm{MN}$ & Special.Sensory & 57 \\
\hline 807 & Laryngeal spasm & 47875 & 64743 & $\mathrm{EC}$ & Respiratory & 53 \\
\hline 808 & Unspecified breast disorder & 6119 & 3244 & $\mathrm{EC}$ & Reproductive.Female & 37 \\
\hline 809 & Other disorders of male genital organs & 60889 & 7537 & $\mathrm{MN}$ & Reproductive.Male & 34 \\
\hline 810 & Actinic keratosis & 7020 & 317644 & $\mathrm{MN}$ & Integumentary & 32 \\
\hline 811 & Concussion with no loss of consciousness & 8500 & 1672 & $\mathrm{MN}$ & Neurologic & 53 \\
\hline 812 & Other and unspecified mycoses & 1179 & 32323 & $\mathrm{MN}$ & Integumentary & 22 \\
\hline 814 & Redness or discharge of eye & 37993 & 37630 & $\mathrm{MN}$ & Special.Sensory & 37 \\
\hline 815 & Syncope and collapse & 7802 & 395926 & $\mathrm{EC}$ & Neurologic & 74 \\
\hline 816 & Blisters, epidermal loss [2nd degree]... & 94520 & 37630 & $\mathrm{MN}$ & Integumentary & 37 \\
\hline 817 & Acute atopic conjunctivitis & 37205 & 62110 & $\mathrm{MN}$ & Special.Sensory & 34 \\
\hline 818 & Abrasion or friction burn of hand exc. . & 9140 & 4041 & $\mathrm{MN}$ & Integumentary & 32 \\
\hline 819 & Other manic-depressive psychosis & 29689 & 30574 & $\mathrm{MN}$ & Psychogenic & 62 \\
\hline 820 & Unspecified functional disorder of st. . . & 5369 & 43545 & $\mathrm{MN}$ & Gastrointestinal & 40 \\
\hline 821 & Raynaud`s syndrome & 4430 & 76298 & $\mathrm{EC}$ & Cardiovascular & 44 \\
\hline 822 & Visual discomfort & 36813 & 6466 & $\mathrm{MN}$ & Special.Sensory & 41 \\
\hline
\end{tabular}




\begin{tabular}{|c|c|c|c|c|c|c|}
\hline Item & ICD-9 Description & ICD-9 Code & $\begin{array}{l}\text { Weighted } \\
\text { Frequency }\end{array}$ & $\begin{array}{l}\text { Decision } \\
\text { Round }\end{array}$ & TPS Category & $\mathrm{IoH}$ \\
\hline 823 & Malignant neoplasm of other specified. & 1728 & 3486 & $\mathrm{EC}$ & Integumentary & 66 \\
\hline 824 & $\begin{array}{l}\text { Carrier or suspected carrier of Methicillin } \\
\text { resistant Staph. . }\end{array}$ & V0254 & 10870 & $\mathrm{EC}$ & Nonspecific & 39 \\
\hline 825 & Unspecified disorder of intestine & 5699 & 6715 & $\mathrm{MN}$ & Gastrointestinal & 34 \\
\hline 826 & Abdominal pain, right lower quadrant & 78903 & 149805 & $\mathrm{MN}$ & Gastrointestinal & 67 \\
\hline 828 & Lipoma of other skin and subcutaneous. . & 2141 & 61888 & $\mathrm{MN}$ & Integumentary & 18 \\
\hline 829 & Influenza with other respiratory mani. . . & 4871 & 702559 & $\mathrm{MN}$ & Respiratory & 53 \\
\hline 830 & Other specified genital prolapse & 61889 & 17594 & $\mathrm{MN}$ & Reproductive.Female & 39 \\
\hline 831 & Sprains and strains of acromioclavicu. & 8400 & 6327 & $\mathrm{MN}$ & Musculoskeletal & 37 \\
\hline 832 & Molluscum contagiosum & 780 & 30648 & $\mathrm{MN}$ & Integumentary & 22 \\
\hline 833 & Urinary incontinence, unspecified & 78830 & 367895 & $\mathrm{EC}$ & Nephrologic & 41 \\
\hline 834 & Brief depressive reaction & 3090 & 224988 & $\mathrm{MN}$ & Psychogenic & 48 \\
\hline 835 & Cardiac dysrhythmia, unspecified & 4279 & 180448 & $\mathrm{MN}$ & Cardiovascular & 64 \\
\hline 836 & Contusion of hip & 92401 & 23894 & $\mathrm{MN}$ & Musculoskeletal & 32 \\
\hline 837 & Sickle-cell anemia, unspecified & 28260 & 4599 & $\mathrm{MN}$ & Hematologic.Immune & 55 \\
\hline 838 & Agoraphobia with panic attacks & 30021 & 14459 & $\mathrm{MN}$ & Psychogenic & 55 \\
\hline 839 & Cerebral artery occlusion, unspec, w/. . & 43490 & 29644 & $\mathrm{EC}$ & Neurologic & 87 \\
\hline 840 & Unspecified disorder of eyelid & 3749 & 11885 & $\mathrm{MN}$ & Special.Sensory & 29 \\
\hline 841 & Ganglion of joint & 72741 & 65891 & $\mathrm{MN}$ & Musculoskeletal & 24 \\
\hline 842 & Unspecified deficiency anemia & 2819 & 28430 & $\mathrm{MN}$ & Hematologic.Immune & 40 \\
\hline 844 & Aftercare following joint replacement & V5481 & 60255 & $\mathrm{MN}$ & Musculoskeletal & 37 \\
\hline 845 & Malignant neoplasm of prostate & 185 & 349067 & $\mathrm{MN}$ & Reproductive.Male & 69 \\
\hline 846 & Foreign body in ear & 931 & 39283 & $\mathrm{MN}$ & Special.Sensory & 41 \\
\hline 847 & Psychosexual dysfunction, unspecified & 30270 & 53602 & $\mathrm{MN}$ & Psychogenic & 44 \\
\hline 848 & Effusion of joint of ankle and foot & 71907 & 40606 & $\mathrm{MN}$ & Musculoskeletal & 44 \\
\hline 849 & Other speech disturbance & 78459 & 11041 & $\mathrm{MN}$ & Neurologic & 51 \\
\hline 850 & Dyschromia, unspecified & 70900 & 35838 & $\mathrm{MN}$ & Integumentary & 20 \\
\hline 851 & Frontal chronic sinusitis & 4731 & 30856 & $\mathrm{MN}$ & Special.Sensory & 39 \\
\hline 853 & Reflux esophagitis & 53011 & 153516 & $\mathrm{MN}$ & Gastrointestinal & 48 \\
\hline 854 & Excercise induced bronchospasm & 49381 & 80945 & $\mathrm{MN}$ & Respiratory & 51 \\
\hline 855 & Other chronic nonalcoholic liver disease & 5718 & 144627 & $\mathrm{MN}$ & Gastrointestinal & 48 \\
\hline 857 & Diseases of lips & 5285 & 73977 & $\mathrm{MN}$ & Integumentary & 29 \\
\hline 858 & Other and unspecified disc disorder, ... & 72291 & 56997 & $\mathrm{MN}$ & Musculoskeletal & 39 \\
\hline 859 & Bipolar affective disorder, unspecified & 2967 & 117815 & $\mathrm{MN}$ & Psychogenic & 60 \\
\hline 860 & Manic-depressive psychosis, unspecified & 29680 & 540672 & $\mathrm{MN}$ & Psychogenic & 64 \\
\hline 862 & Chronic kidney disease, unspecified & 5859 & 456096 & $\mathrm{MN}$ & Nephrologic & 57 \\
\hline 863 & Closed dislocation of hip, unspecified & 83500 & 25425 & $\mathrm{MN}$ & Musculoskeletal & 67 \\
\hline 864 & Agoraphobia without mention of panic ... & 30022 & 6911 & $\mathrm{MN}$ & Psychogenic & 51 \\
\hline 865 & $\begin{array}{l}\text { Dementia, unspec, w/o behavioral } \\
\text { disturbance }\end{array}$ & 29420 & 459859 & $\mathrm{MN}$ & Neurologic & 60 \\
\hline 866 & Contusion of chest wall & 9221 & 230003 & $\mathrm{MN}$ & Musculoskeletal & 27 \\
\hline 868 & Orchitis and epididymitis, unspecified & 60490 & 138769 & $\mathrm{MN}$ & Reproductive.Male & 53 \\
\hline 869 & Chronic kidney disease, stage $\mathrm{V}$ & 5855 & 6783 & $\mathrm{MN}$ & Nephrologic & 72 \\
\hline 871 & Cyst of Bartholin`s gland & 6162 & 10426 & $\mathrm{MN}$ & Reproductive.Female & 37 \\
\hline 872 & Closed fracture of carpal bone, unspe. . . & 81400 & 53856 & $\mathrm{MN}$ & Musculoskeletal & 55 \\
\hline 874 & Irregular menstrual cycle & 6264 & 151578 & $\mathrm{MN}$ & Reproductive.Female & 46 \\
\hline 875 & Multiple sclerosis & 340 & 213549 & $\mathrm{EC}$ & Neurologic & 64 \\
\hline 876 & Major depressive disorder, recurrent ... & 29636 & 14219 & $\mathrm{MN}$ & Psychogenic & 62 \\
\hline 877 & Intrauterine contraceptive device & V2542 & 51797 & $\mathrm{MN}$ & Reproductive.Female & 41 \\
\hline 878 & Colitis, enteritis, \& gastroenteritis... & 91 & 28430 & $\mathrm{MN}$ & Gastrointestinal & 55 \\
\hline
\end{tabular}

Continued 


\begin{tabular}{|c|c|c|c|c|c|c|}
\hline Item & ICD-9 Description & ICD-9 Code & $\begin{array}{l}\text { Weighted } \\
\text { Frequency }\end{array}$ & $\begin{array}{c}\text { Decision } \\
\text { Round }\end{array}$ & TPS Category & $\mathrm{IoH}$ \\
\hline 879 & Counseling on substance use and abuse & V6542 & 5798 & $\mathrm{EC}$ & Psychogenic & 48 \\
\hline 880 & Unspecified endocrine disorder & 2599 & 14472 & $\mathrm{MN}$ & Endocrine & 32 \\
\hline 882 & Synovial cyst of popliteal space & 72751 & 14647 & $\mathrm{MN}$ & Musculoskeletal & 29 \\
\hline 883 & Phobia, unspecified & 30020 & 14372 & $\mathrm{MN}$ & Psychogenic & 37 \\
\hline 884 & Closed fracture of base of other meta... & 81502 & 28852 & $\mathrm{MN}$ & Musculoskeletal & 46 \\
\hline 885 & Other acute pain & 33819 & 124644 & $\mathrm{MN}$ & Nonspecific & 41 \\
\hline 886 & Other benign neoplasm of site unspeci. . & 2159 & 11643 & $\mathrm{MN}$ & Nonspecific & 24 \\
\hline 887 & Other forms of migraine without menti... & 34680 & 40002 & $\mathrm{MN}$ & Neurologic & 48 \\
\hline 888 & Unspecif hypertensive heart disease w. . . & 40291 & 3185 & $\mathrm{MN}$ & Cardiovascular & 64 \\
\hline 889 & Long-term (current) use of insulin & V5867 & 177370 & $\mathrm{MN}$ & Endocrine & 60 \\
\hline 892 & Open wound of wrist, without mention ... & 88102 & 11414 & EC & Integumentary & 37 \\
\hline 893 & Nonproliferative diabetic retinopathy. . . & 36203 & 32442 & $\mathrm{EC}$ & Special.Sensory & 57 \\
\hline 894 & Other specified local infections of s... & 6868 & 3953 & $\mathrm{MN}$ & Integumentary & 39 \\
\hline 895 & Unspecified disease of pericardium & 4239 & 28890 & $\mathrm{MN}$ & Cardiovascular & 60 \\
\hline 897 & Ot/uns intrac hem folow inj w/o men o... & 85300 & 10250 & $\mathrm{MN}$ & Neurologic & 73 \\
\hline 898 & Attention deficit disorder without me... & 31400 & 1695114 & EC & Psychogenic & 48 \\
\hline 899 & Unspecified disorders of nervous system & 3499 & 34726 & $\mathrm{MN}$ & Neurologic & 39 \\
\hline 900 & Abdominal pain, left lower quadrant & 78904 & 135815 & $\mathrm{MN}$ & Gastrointestinal & 60 \\
\hline 901 & Unspecified intracranial hemorrhage & 4329 & 27114 & $\mathrm{MN}$ & Neurologic & 95 \\
\hline 902 & Systolic heart failure, acute & 42821 & 17385 & $\mathrm{MN}$ & Cardiovascular & 96 \\
\hline 903 & Acquired acanthosis nigricans & 7012 & 28430 & $\mathrm{MN}$ & Integumentary & 32 \\
\hline 904 & Routine infant or child health check & V202 & 3981438 & $\mathrm{EC}$ & Patient.based.Systems & 48 \\
\hline 905 & Acute tracheitis without mention of o... & 46410 & 25279 & $\mathrm{EC}$ & Respiratory & 62 \\
\hline 906 & Effusion of joint of hand & 71904 & 8916 & $\mathrm{MN}$ & Musculoskeletal & 44 \\
\hline 907 & Tenosynovitis of foot and ankle & 72706 & 12776 & $\mathrm{MN}$ & Musculoskeletal & 41 \\
\hline 908 & Mixed hyperlipidemia & 2722 & 1567589 & $\mathrm{MN}$ & Cardiovascular & 48 \\
\hline 909 & Personal history of tuberculosis & V1201 & 17594 & $\mathrm{EC}$ & Respiratory & 44 \\
\hline 910 & Examination following other treatment & V6759 & 2850911 & $\mathrm{MN}$ & Nonspecific & 15 \\
\hline 911 & Generalized convulsv epilepsy w/o men... & 34510 & 16453 & $\mathrm{MN}$ & Neurologic & 64 \\
\hline 912 & Ankylosing spondylitis & 7200 & 26239 & $\mathrm{MN}$ & Musculoskeletal & 53 \\
\hline 913 & Burn of upper arm, unspecified degree & 94303 & 15980 & $\mathrm{MN}$ & Integumentary & 39 \\
\hline 914 & Benign neoplasm of skin, site unspeci. . . & 2169 & 371588 & $\mathrm{MN}$ & Integumentary & 29 \\
\hline 915 & Vomiting alone & 78703 & 359225 & $\mathrm{MN}$ & Gastrointestinal & 37 \\
\hline 916 & Diastolic heart failure, unspecified & 42830 & 28430 & $\mathrm{MN}$ & Cardiovascular & 57 \\
\hline 917 & Family hx of malignant neoplasm prostate & V1642 & 7643 & $\mathrm{MN}$ & Reproductive.Male & 37 \\
\hline 918 & Other anterior pituitary disorders & 2534 & 11161 & $\mathrm{MN}$ & Endocrine & 44 \\
\hline 919 & Disability examination & V6801 & 17385 & $\mathrm{EC}$ & Patient.based.Systems & 39 \\
\hline 920 & Hemangioma of skin and subcutaneous t. . . & 22801 & 28430 & $\mathrm{MN}$ & Integumentary & 27 \\
\hline 921 & Other conjunctivitis & 37239 & 7828 & $\mathrm{MN}$ & Special.Sensory & 24 \\
\hline 922 & Uterine prolapse without mention of $\mathrm{v} . \ldots$ & 6181 & 17594 & $\mathrm{MN}$ & Reproductive.Female & 41 \\
\hline 923 & Acute lymphadenitis & 683 & 58100 & $\mathrm{MN}$ & Hematologic.Immune & 44 \\
\hline 924 & Polyglandular dysfunction, unspecified & 2589 & 28430 & $\mathrm{MN}$ & Endocrine & 41 \\
\hline 925 & Brachia neuritis or radiculitis NOS & 7234 & 372064 & $\mathrm{EC}$ & Neurologic & 46 \\
\hline 926 & Other joint derangement of pelvic reg. . & 71885 & 17594 & $\mathrm{MN}$ & Musculoskeletal & 37 \\
\hline 927 & Neoplasm of uncertain behav of pituit. . & 2370 & 26368 & $\mathrm{MN}$ & Endocrine & 55 \\
\hline 928 & Unspecified hypothyroidism & 2449 & 5055098 & $\mathrm{MN}$ & Endocrine & 48 \\
\hline 930 & Retention of urine, unspecified & 78820 & 61660 & $\mathrm{EC}$ & Nephrologic & 47 \\
\hline
\end{tabular}




\begin{tabular}{|c|c|c|c|c|c|c|}
\hline Item & ICD-9 Description & ICD-9 Code & $\begin{array}{l}\text { Weighted } \\
\text { Frequency }\end{array}$ & $\begin{array}{l}\text { Decision } \\
\text { Round }\end{array}$ & TPS Category & $\mathrm{IoH}$ \\
\hline 931 & Hypersomnia with sleep apnea & 78053 & 46806 & $\mathrm{EC}$ & Respiratory & 60 \\
\hline 933 & Closed fracture of one or more phalan. . . & 8260 & 34227 & $\mathrm{MN}$ & Musculoskeletal & 39 \\
\hline 934 & Neoplasm of uncertain behavior of oth. & 2388 & 7437 & $\mathrm{MN}$ & Nonspecific & 52 \\
\hline 935 & Benign paroxysmal positional vertigo & 38611 & 98630 & $\mathrm{EC}$ & Neurologic & 44 \\
\hline 936 & Candidiasis of vulva and vagina & 1121 & 345578 & $\mathrm{MN}$ & Reproductive.Female & 39 \\
\hline 937 & Contusion of abdominal wall & 9222 & 23403 & $\mathrm{EC}$ & Musculoskeletal & 34 \\
\hline 938 & Personal history of other diseases of. . . & V1279 & 118328 & $\mathrm{EC}$ & Gastrointestinal & 29 \\
\hline 939 & Sprains and strains of other area of ... & 84519 & 3875 & $\mathrm{MN}$ & Musculoskeletal & 27 \\
\hline 940 & Other hammer toe (acquired) & 7354 & 14680 & $\mathrm{MN}$ & Musculoskeletal & 27 \\
\hline 941 & Unspecified disorder of adrenal glands & 2559 & 25985 & $\mathrm{MN}$ & Endocrine & 46 \\
\hline 943 & Unspecified disorder of synovium, ten. . . & 7279 & 16692 & $\mathrm{MN}$ & Musculoskeletal & 34 \\
\hline 944 & Unspecified deformity of forearm, exc. . & 73600 & 17148 & $\mathrm{MN}$ & Musculoskeletal & 37 \\
\hline 945 & Medullary sponge kidney & 75317 & 8382 & $\mathrm{MN}$ & Nephrologic & 48 \\
\hline 946 & Crushing injury of foot & 92820 & 28062 & $\mathrm{MN}$ & Musculoskeletal & 64 \\
\hline 947 & Precocious sexual development and pub... & 2591 & 31599 & $\mathrm{MN}$ & Endocrine & 57 \\
\hline 948 & Aortic ectasia, unspecified site & 44770 & 19549 & $\mathrm{MN}$ & Cardiovascular & 51 \\
\hline 949 & Osteopathy resulting from poliomyelit. . . & 73076 & 27114 & $\mathrm{MN}$ & Musculoskeletal & 41 \\
\hline 951 & Unspecified retinal detachment & 3619 & 11885 & $\mathrm{MN}$ & Special.Sensory & 72 \\
\hline 953 & Hemoptysis, unspecified & 78630 & 35136 & $\mathrm{MN}$ & Respiratory & 60 \\
\hline 954 & Unspecified paranoid state & 2979 & 19826 & $\mathrm{MN}$ & Psychogenic & 57 \\
\hline 955 & Atherosclerosis of the extremities, u. . . & 44020 & 17594 & $\mathrm{MN}$ & Cardiovascular & 53 \\
\hline 956 & Choanal atresia & 7480 & 7367 & $\mathrm{EC}$ & Respiratory & 51 \\
\hline 957 & Opn wnd of abdominl wall, anterior, w. . . & 8792 & 20520 & $\mathrm{MN}$ & Integumentary & 48 \\
\hline 958 & Special screening for osteoporosis & V8281 & 14372 & $\mathrm{EC}$ & Musculoskeletal & 37 \\
\hline 959 & Neoplasm of uncertain behavior of skin & 2382 & 180476 & $\mathrm{MN}$ & Integumentary & 46 \\
\hline 960 & Paralysis agitans & 3320 & 269305 & $\mathrm{EC}$ & Neurologic & 53 \\
\hline 961 & Other specified anomalies of skin & 75739 & 41019 & $\mathrm{MN}$ & Integumentary & 24 \\
\hline 962 & $\begin{array}{l}\text { Cervical high risk human papillomavirus[HPV] } \\
\text { DNA test positive }\end{array}$ & 79505 & 15464 & $\mathrm{MN}$ & Reproductive.Female & 57 \\
\hline 963 & Episodic cluster headache & 33901 & 10334 & $\mathrm{MN}$ & Neurologic & 53 \\
\hline 964 & Insect bite of foot and toe(s), nonve... & 9174 & 14823 & $\mathrm{MN}$ & Integumentary & 22 \\
\hline 965 & Nevus, non-neoplastic & 4481 & 42127 & $\mathrm{MN}$ & Integumentary & 27 \\
\hline 966 & Diabetes mellitus w/o complication, t. . & 25001 & 223460 & $\mathrm{MN}$ & Endocrine & 53 \\
\hline 967 & Abdominal pain, unspecified site & 78900 & 2420935 & $\mathrm{MN}$ & Gastrointestinal & 53 \\
\hline 968 & Secondary neuroendocrine tumor, unspec site & 20970 & 7043 & $\mathrm{MN}$ & Endocrine & 57 \\
\hline 969 & Diseases of tricuspid valve & 3970 & 16002 & $\mathrm{MN}$ & Cardiovascular & 53 \\
\hline 970 & Prescription of oral contraceptives & V2501 & 6921 & $\mathrm{MN}$ & Reproductive.Female & 41 \\
\hline 971 & Specl screeng for oth spec mental dis... & V798 & 16002 & $\mathrm{EC}$ & Psychogenic & 37 \\
\hline 972 & Scanty or infrequent menstruation & 6261 & 21032 & $\mathrm{MN}$ & Reproductive.Female & 44 \\
\hline 975 & Pain in or around eye & 37991 & 38363 & $\mathrm{MN}$ & Special.Sensory & 46 \\
\hline 976 & Solitary pulmonary nodule & 79311 & 20920 & $\mathrm{MN}$ & Respiratory & 55 \\
\hline 978 & Full incontinence of feces & 78760 & 32466 & $\mathrm{MN}$ & Gastrointestinal & 51 \\
\hline 979 & Other left bundle branch block & 4263 & 6652 & $\mathrm{MN}$ & Cardiovascular & 46 \\
\hline 981 & Malignant neoplasm of bladder, part u. . . & 1889 & 45059 & $\mathrm{EC}$ & Nephrologic & 69 \\
\hline 982 & Description not found & V998 & 14372 & $\mathrm{MN}$ & Nonspecific & 16 \\
\hline 983 & Rectocele & 61804 & 24095 & $\mathrm{EC}$ & Gastrointestinal & 39 \\
\hline 984 & Abdominal pain, left upper quadrant & 78902 & 49383 & $\mathrm{MN}$ & Gastrointestinal & 57 \\
\hline 985 & Personal history Venous thrombosis and embolism & V1251 & 24095 & $\mathrm{EC}$ & Cardiovascular & 46 \\
\hline 986 & Myoclonus & 3332 & 13961 & $\mathrm{MN}$ & Neurologic & 44 \\
\hline 987 & Tarsal tunnel syndrome & 3555 & 16224 & $\mathrm{MN}$ & Musculoskeletal & 39 \\
\hline
\end{tabular}




\begin{tabular}{|c|c|c|c|c|c|c|}
\hline Item & ICD-9 Description & ICD-9 Code & $\begin{array}{l}\text { Weighted } \\
\text { Frequency }\end{array}$ & $\begin{array}{c}\text { Decision } \\
\text { Round }\end{array}$ & TPS Category & $\mathrm{IoH}$ \\
\hline 988 & Cellulitis and abscess of unspecified... & 6819 & 51585 & $\mathrm{MN}$ & Integumentary & 48 \\
\hline 990 & Hematuria, unspecified & 59970 & 521389 & $\mathrm{MN}$ & Nephrologic & 53 \\
\hline 991 & Closed fracture of rib(s), unspecified & 80700 & 35929 & $\mathrm{MN}$ & Musculoskeletal & 41 \\
\hline 992 & Heart disease, unspecified & 4299 & 50385 & $\mathrm{MN}$ & Cardiovascular & 51 \\
\hline 993 & Other and unspecified diseases of upp. . & 4789 & 34761 & $\mathrm{EC}$ & Respiratory & 23 \\
\hline 994 & Enthesopathy of ankle and tarsus, uns... & 72670 & 87201 & $\mathrm{MN}$ & Musculoskeletal & 29 \\
\hline 995 & Systolic heart failure, chronic & 42822 & 56859 & $\mathrm{MN}$ & Cardiovascular & 67 \\
\hline 996 & Enlargement of lymph nodes & 7856 & 310843 & $\mathrm{MN}$ & Hematologic.Immune & 51 \\
\hline 997 & Lateral epicondylitis & 72632 & 365636 & $\mathrm{MN}$ & Musculoskeletal & 29 \\
\hline 998 & Ganglion, unspecified & 72743 & 62150 & $\mathrm{MN}$ & Musculoskeletal & 22 \\
\hline 1000 & Superfic frgn body of foot/toe w/o op. . . & 9176 & 8004 & $\mathrm{EC}$ & Integumentary & 34 \\
\hline 1001 & Intestinovesical fistula & 5961 & 3064 & $\mathrm{MN}$ & Gastrointestinal & 65 \\
\hline 1002 & Neoplasms of unspecified nature brain & 2396 & 3591 & $\mathrm{MN}$ & Neurologic & 71 \\
\hline 1003 & Late effect of unspecified injury & 9089 & 62046 & $\mathrm{MN}$ & Nonspecific & 31 \\
\hline 1004 & Psychosexual dysfunction with oth spe... & 30279 & 27114 & $\mathrm{EC}$ & Psychogenic & 40 \\
\hline 1005 & Flat foot & 734 & 50597 & $\mathrm{MN}$ & Musculoskeletal & 20 \\
\hline 1006 & Other specified aftercare & V5889 & 2217235 & $\mathrm{MN}$ & Nonspecific & 24 \\
\hline 1007 & Unspecified hearing loss & 3899 & 245656 & $\mathrm{MN}$ & Special.Sensory & 37 \\
\hline 1008 & Intest disaccharidase deficiencies \& ... & 2713 & 110029 & $\mathrm{MN}$ & Gastrointestinal & 40 \\
\hline 1009 & Aftercare traumatic fract of arm, unspec & V5410 & 17572 & $\mathrm{MN}$ & Musculoskeletal & 34 \\
\hline 1010 & Contact dermatitis/eczema of due to d... & 6920 & 54227 & $\mathrm{MN}$ & Integumentary & 32 \\
\hline 1011 & Essential thrombocythemia & 23871 & 11161 & $\mathrm{MN}$ & Hematologic.Immune & 45 \\
\hline 1012 & Radial styloid tenosynovitis & 72704 & 36230 & $\mathrm{MN}$ & Musculoskeletal & 32 \\
\hline 1013 & Diabetes w/neurological manifestation. . . & 25063 & 17385 & $\mathrm{MN}$ & Endocrine & 57 \\
\hline 1014 & Other and unspecified coagulation def. . & 2869 & 58407 & $\mathrm{MN}$ & Hematologic.Immune & 47 \\
\hline 1015 & Other cellulitis and abscess of hand,... & 6824 & 1522 & $\mathrm{EC}$ & Integumentary & 47 \\
\hline 1017 & Torticollis, unspecified & 7235 & 109853 & $\mathrm{MN}$ & Musculoskeletal & 40 \\
\hline 1018 & Chronic hepatitis, unspecified & 57140 & 28045 & $\mathrm{MN}$ & Gastrointestinal & 45 \\
\hline 1019 & Closed fracture of lower end, unspeci... & 81240 & 37630 & $\mathrm{MN}$ & Musculoskeletal & 47 \\
\hline 1020 & Appendicitis, unqualified & 541 & 15749 & $\mathrm{MN}$ & Gastrointestinal & 77 \\
\hline 1022 & Other specified disorders of esophagus & 53089 & 12647 & $\mathrm{MN}$ & Gastrointestinal & 37 \\
\hline 1023 & Tracheostomy & V440 & 10250 & $\mathrm{EC}$ & Respiratory & 45 \\
\hline 1024 & Plantar fascial fibromatosis & 72871 & 486371 & $\mathrm{MN}$ & Musculoskeletal & 32 \\
\hline 1025 & Other symptoms involving nervous and ... & 78199 & 52294 & $\mathrm{MN}$ & Neurologic & 32 \\
\hline 1026 & Benign neoplasm of skin of trunk, exc. . & 2165 & 41116 & $\mathrm{MN}$ & Integumentary & 23 \\
\hline 1027 & Human immunodeficiency virus (HIV) di... & 42 & 69902 & $\mathrm{MN}$ & Hematologic.Immune & 65 \\
\hline 1028 & Herpes zoster with other opthalmic co... & 5329 & 37630 & $\mathrm{EC}$ & Special.Sensory & 71 \\
\hline 1029 & Adhesive capsulitis of shoulder & 7260 & 3897 & $\mathrm{MN}$ & Musculoskeletal & 42 \\
\hline 1030 & Other specified urticaria & 7088 & 24502 & $\mathrm{EC}$ & Integumentary & 40 \\
\hline 1031 & Trunk, other injury of chest wall & 95911 & 53769 & $\mathrm{MN}$ & Musculoskeletal & 37 \\
\hline 1032 & Unspecif gastritis/gastroduodenitis w. . . & 53550 & 435104 & $\mathrm{MN}$ & Gastrointestinal & 42 \\
\hline 1033 & Closed dislocation of elbow unspecified & 83200 & 3307 & $\mathrm{MN}$ & Musculoskeletal & 52 \\
\hline 1034 & Obsessive-compulsive disorders & 3003 & 111524 & $\mathrm{MN}$ & Psychogenic & 52 \\
\hline 1035 & Poisoning by electrolytic, caloric, a. . & 9745 & 37630 & $\mathrm{EC}$ & Nonspecific & 67 \\
\hline 1036 & Prophylactic vaccination/inoculation ... & V032 & 12081 & $\mathrm{MN}$ & Population.based.Care & 45 \\
\hline 1037 & Hydrocele, unspecified & 6039 & 35188 & $\mathrm{MN}$ & Reproductive.Male & 37 \\
\hline 1039 & Maxillary acute sinusitis & 4610 & 308842 & $\mathrm{EC}$ & Respiratory & 42 \\
\hline 1040 & Long-term (current) use of steroids & V5865 & 1830 & $\mathrm{EC}$ & Nonspecific & 47 \\
\hline 1041 & Unspecified drug dependence, unspecified & 30490 & 61872 & $\mathrm{MN}$ & Psychogenic & 52 \\
\hline 1042 & Impotence of organic origin & 60784 & 766755 & $\mathrm{MN}$ & Reproductive.Male & 45 \\
\hline
\end{tabular}




\begin{tabular}{|c|c|c|c|c|c|c|}
\hline Item & ICD-9 Description & ICD-9 Code & $\begin{array}{l}\text { Weighted } \\
\text { Frequency }\end{array}$ & $\begin{array}{l}\text { Decision } \\
\text { Round }\end{array}$ & TPS Category & $\mathrm{IoH}$ \\
\hline 1043 & Health examination of defined subpopu. . . & V705 & 668771 & $\mathrm{MN}$ & Population.based.Care & 37 \\
\hline 1044 & Other lymphatic and hematopoietic tissue... & 23879 & 8984 & $\mathrm{MN}$ & Hematologic.Immune & 40 \\
\hline 1045 & Infantile autism, current or active s... & 29900 & 62548 & $\mathrm{EC}$ & Psychogenic & 55 \\
\hline 1046 & Polycystic ovaries & 2564 & 42707 & $\mathrm{MN}$ & Reproductive.Female & 47 \\
\hline 1047 & Other specified administrative purpose & V6889 & 66191 & $\mathrm{MN}$ & Nonspecific & 1 \\
\hline 1048 & Other symptoms referable to joint of. & 71966 & 6192 & $\mathrm{MN}$ & Musculoskeletal & 26 \\
\hline 1049 & Lumbosacral spondylosis without myelo... & 7213 & 231466 & $\mathrm{MN}$ & Musculoskeletal & 32 \\
\hline 1050 & Grand mal status & 3453 & 17385 & $\mathrm{MN}$ & Neurologic & 74 \\
\hline 1051 & Exam followg treatmt w/high-risk medi. & V6751 & 28430 & $\mathrm{MN}$ & Nonspecific & 40 \\
\hline 1052 & Immune thrombocytopenic purpura & 28731 & 61178 & $\mathrm{MN}$ & Hematologic.Immune & 57 \\
\hline 1053 & Examination of eyes and vision & V720 & 79425 & $\mathrm{MN}$ & Special.Sensory & 32 \\
\hline 1054 & Arthropathy, unspecified, upper arm & 71692 & 3079 & $\mathrm{MN}$ & Musculoskeletal & 32 \\
\hline 1055 & Other specified dermatoses & 7028 & 4426 & $\mathrm{MN}$ & Integumentary & 26 \\
\hline 1056 & Sprains and strains of other specifie... & 8438 & 34275 & $\mathrm{MN}$ & Musculoskeletal & 32 \\
\hline 1057 & Bladder neck obstruction & 5960 & 25180 & $\mathrm{EC}$ & Nephrologic & 55 \\
\hline 1058 & $\begin{array}{l}\text { Eosinophilic gastritis, w/o ment of } \\
\text { hemorrhage }\end{array}$ & 53570 & 18850 & $\mathrm{MN}$ & Gastrointestinal & 47 \\
\hline 1059 & Rosacea conjunctivitis & 37231 & 7691 & $\mathrm{EC}$ & Special.Sensory & 34 \\
\hline 1060 & Abrasion or friction burn of hand exc... & 9141 & 46806 & $\mathrm{MN}$ & Integumentary & 34 \\
\hline 1061 & Hemophthalmos, except current injury & 36043 & 5159 & EC & Special.Sensory & 52 \\
\hline 1062 & Sebaceous cyst & 7062 & 667439 & $\mathrm{MN}$ & Integumentary & 23 \\
\hline 1063 & Juvenile osteochondrosis of lower ext. . & 7324 & 2568 & $\mathrm{MN}$ & Musculoskeletal & 45 \\
\hline 1064 & Osteoarthrosis, unspec generalized or. . & 71598 & 8004 & $\mathrm{MN}$ & Musculoskeletal & 34 \\
\hline 1065 & Supervision of normal first pregnancy & V220 & 73902 & $\mathrm{MN}$ & Reproductive.Female & 55 \\
\hline 1066 & Initiation of other contraceptive mea. . & V2502 & 10134 & $\mathrm{MN}$ & Reproductive.Female & 50 \\
\hline 1067 & Closed dislocation of shoulder, unspe... & 83100 & 2491 & $\mathrm{MN}$ & Musculoskeletal & 57 \\
\hline 1069 & Other urinary problems & V474 & 17594 & $\mathrm{EC}$ & Nephrologic & 34 \\
\hline 1070 & Candidiasis of skin and nails & 1123 & 64560 & $\mathrm{MN}$ & Integumentary & 29 \\
\hline 1071 & True post wall infarc of othr spec si... & 41080 & 8137 & $\mathrm{MN}$ & Cardiovascular & 81 \\
\hline 1073 & Other disease of nasal cavity and sinuse... & 47819 & 718867 & $\mathrm{EC}$ & Respiratory & 34 \\
\hline 1074 & Goiter, unspecified & 2409 & 147983 & $\mathrm{MN}$ & Endocrine & 47 \\
\hline 1075 & Undifferentiated somatoform disorder & 30082 & 26368 & $\mathrm{MN}$ & Psychogenic & 42 \\
\hline 1076 & Blisters/epidermal loss [second degre... & 94127 & 7818 & $\mathrm{MN}$ & Integumentary & 40 \\
\hline 1077 & Vesical fistula, not elsewhere classi... & 5962 & 12770 & $\mathrm{EC}$ & Nephrologic & 52 \\
\hline 1078 & Underimmunization status & V1583 & 5946 & $\mathrm{MN}$ & Population.based.Care & 42 \\
\hline 1079 & Fecal smearing & 78762 & 3591 & $\mathrm{MN}$ & Gastrointestinal & 26 \\
\hline 1080 & Malignant neoplasm of appendix & 1535 & 3798 & $\mathrm{MN}$ & Gastrointestinal & 77 \\
\hline 1081 & Tension headache & 30781 & 173230 & $\mathrm{MN}$ & Neurologic & 47 \\
\hline 1083 & Osteoarthrosis, unspec generalized or. . & 71597 & 35297 & $\mathrm{MN}$ & Musculoskeletal & 34 \\
\hline 1085 & Other cellulitis and abscess of buttock & 6825 & 24342 & $\mathrm{MN}$ & Integumentary & 50 \\
\hline 1086 & Dysphagia & 43882 & 17871 & $\mathrm{MN}$ & Gastrointestinal & 57 \\
\hline 1087 & Diabetes mellitus, antepartum conditi. . & 64803 & 24620 & $\mathrm{MN}$ & Endocrine & 63 \\
\hline 1088 & Urinary tract infection, site not spe... & 5990 & 2752888 & $\mathrm{EC}$ & Nephrologic & 45 \\
\hline 1090 & Hypertrophy of nasal turbinates & 4780 & 7935 & $\mathrm{EC}$ & Respiratory & 29 \\
\hline 1091 & Examination following surgery, unspecif & V6700 & 171128 & $\mathrm{MN}$ & Nonspecific & 28 \\
\hline 1092 & Nausea with vomiting & 78701 & 243106 & $\mathrm{MN}$ & Gastrointestinal & 47 \\
\hline 1093 & Other symptoms involving urinary system & 78899 & 186597 & $\mathrm{EC}$ & Nephrologic & 37 \\
\hline 1094 & Backache, unspecified & 7245 & 3661663 & $\mathrm{MN}$ & Musculoskeletal & 45 \\
\hline 1095 & Overweight & 27802 & 605840 & $\mathrm{EC}$ & Nonspecific & 50 \\
\hline
\end{tabular}




\begin{tabular}{|c|c|c|c|c|c|c|}
\hline Item & ICD-9 Description & ICD-9 Code & $\begin{array}{l}\text { Weighted } \\
\text { Frequency }\end{array}$ & $\begin{array}{l}\text { Decision } \\
\text { Round }\end{array}$ & TPS Category & $\mathrm{IoH}$ \\
\hline 1096 & Abdominal pain, periumbilic & 78905 & 29332 & $\mathrm{MN}$ & Gastrointestinal & 52 \\
\hline 1097 & Barbiturate/simil actg sedative/hypno... & 30410 & 3466 & $\mathrm{EC}$ & Psychogenic & 48 \\
\hline 1098 & Open wound of ear drum & 87271 & 5648 & $\mathrm{MN}$ & Special.Sensory & 47 \\
\hline 1099 & Brachial plexus lesions & 3530 & 24141 & $\mathrm{MN}$ & Neurologic & 55 \\
\hline 1100 & Hepatitis, unspecified & 5733 & 109551 & $\mathrm{MN}$ & Gastrointestinal & 55 \\
\hline 1101 & Dermatophytosis of nail & 1101 & 331627 & $\mathrm{MN}$ & Integumentary & 16 \\
\hline 1102 & Other primary cardiomyopathies & 4254 & 228747 & $\mathrm{MN}$ & Cardiovascular & 68 \\
\hline 1103 & Special screening for thyroid disorders & V770 & 40314 & $\mathrm{MN}$ & Endocrine & 34 \\
\hline 1104 & Other specified symptoms associated w. . & 6258 & 58024 & $\mathrm{EC}$ & Reproductive.Female & 30 \\
\hline 1105 & Other specified examination & V7285 & 176447 & $\mathrm{MN}$ & Nonspecific & 18 \\
\hline 1106 & Hordeolum externum & 37311 & 103601 & $\mathrm{EC}$ & Special.Sensory & 26 \\
\hline 1107 & Osteoarthrosis, unspec generalized or. . & 71595 & 215806 & $\mathrm{MN}$ & Musculoskeletal & 34 \\
\hline 1109 & Rhesus isoimmunization, antepartum co... & 65613 & 11942 & $\mathrm{EC}$ & Hematologic.Immune & 52 \\
\hline 1110 & Abrasion or friction burn without men. . & 9100 & 40230 & $\mathrm{MN}$ & Integumentary & 23 \\
\hline 1111 & Viral exanthem, unspecified & 579 & 20690 & $\mathrm{MN}$ & Integumentary & 32 \\
\hline 1112 & Muscular wasting and disuse atrophy, ... & 7282 & 10732 & $\mathrm{MN}$ & Musculoskeletal & 52 \\
\hline 1113 & Issue of repeat prescriptions & V681 & 464378 & $\mathrm{EC}$ & Patient.based.Systems & 29 \\
\hline 1114 & Other specified perinatal disorders o... & 7778 & 7831 & $\mathrm{MN}$ & Reproductive.Female & 33 \\
\hline 1115 & Acute laryngitis wo mention of obstructn & 46400 & 174971 & $\mathrm{EC}$ & Respiratory & 37 \\
\hline 1116 & Abrasion/friction burn of oth/multipl. . . & 9190 & 76088 & $\mathrm{MN}$ & Integumentary & 29 \\
\hline 1117 & Pain in thoracic spine & 7241 & 425859 & $\mathrm{MN}$ & Musculoskeletal & 34 \\
\hline 1118 & $\begin{array}{l}\text { Personal history of transient ischemic attack } \\
\text { and cereb.. }\end{array}$ & V1254 & 108190 & $\mathrm{MN}$ & Neurologic & 57 \\
\hline 1119 & Contusion of toe & 9243 & 58858 & $\mathrm{MN}$ & Musculoskeletal & 16 \\
\hline 1120 & Inguinal hernia, without obstruction ... & 55092 & 10432 & $\mathrm{EC}$ & Gastrointestinal & 45 \\
\hline 1121 & Other disorders of ear & 3888 & 44611 & $\mathrm{MN}$ & Special.Sensory & 31 \\
\hline 1122 & Retinal vascular occlusion, unspecified & 36230 & 17594 & $\mathrm{MN}$ & Special.Sensory & 71 \\
\hline 1123 & Anomaly of the peripheral vascular sy... & 74760 & 7480 & $\mathrm{MN}$ & Cardiovascular & 52 \\
\hline 1124 & Intestinal infection due to other ana... & 846 & 6348 & $\mathrm{MN}$ & Gastrointestinal & 52 \\
\hline 1125 & Sprains and strains of unspecified si... & 84510 & 95852 & $\mathrm{MN}$ & Musculoskeletal & 23 \\
\hline 1126 & Abdominal or pelvic swelling, mass, o... & 78939 & 8187 & $\mathrm{MN}$ & Gastrointestinal & 55 \\
\hline 1127 & Classical migraine without mention of. . & 34600 & 109673 & $\mathrm{MN}$ & Neurologic & 50 \\
\hline 1128 & Neoplasms of unspecified nature diges. . & 2390 & 28430 & $\mathrm{MN}$ & Gastrointestinal & 60 \\
\hline 1129 & Obesity, unspecified & 27800 & 3835783 & $\mathrm{EC}$ & Nonspecific & 52 \\
\hline 1130 & Personal history of diseases of skin ... & V133 & 1985 & $\mathrm{MN}$ & Integumentary & 32 \\
\hline 1131 & Chronic laryngitis & 4760 & 5100 & $\mathrm{EC}$ & Respiratory & 40 \\
\hline 1132 & Occlusion and stenosis of carotid art... & 43311 & 28430 & $\mathrm{MN}$ & Cardiovascular & 68 \\
\hline 1133 & Causalgia & 3544 & 46806 & $\mathrm{MN}$ & Nonspecific & 35 \\
\hline 1134 & Aortocoronary bypass status & V4581 & 11942 & $\mathrm{MN}$ & Cardiovascular & 57 \\
\hline 1135 & Desensitization to allergens & V071 & 56135 & $\mathrm{MN}$ & Hematologic.Immune & 37 \\
\hline 1136 & Multiple and unspecified open wound o... & 8841 & 18491 & $\mathrm{MN}$ & Integumentary & 45 \\
\hline 1137 & Tachypnea & 78606 & 36257 & $\mathrm{MN}$ & Respiratory & 63 \\
\hline 1138 & Benign essential hypertension & 4011 & 4744514 & $\mathrm{MN}$ & Cardiovascular & 50 \\
\hline 1139 & Closed fracture of metacarpal bone(s)... & 81500 & 54931 & $\mathrm{MN}$ & Musculoskeletal & 40 \\
\hline 1140 & Cardiomegaly & 4293 & 35910 & $\mathrm{MN}$ & Cardiovascular & 63 \\
\hline 1141 & $\begin{array}{l}\text { Basal cell carcinoma skin upper limb, } \\
\text { including shoulder }\end{array}$ & 17361 & 5697 & $\mathrm{MN}$ & Integumentary & 57 \\
\hline 1142 & Generalized anxiety disorder & 30002 & 808730 & $\mathrm{MN}$ & Psychogenic & 47 \\
\hline 1143 & Diabetes w/other spec manifestations,... & 25082 & 27114 & $\mathrm{MN}$ & Endocrine & 57 \\
\hline 1144 & Hypoglycemia, unspecified & 2512 & 137572 & $\mathrm{MN}$ & Endocrine & 57 \\
\hline
\end{tabular}




\begin{tabular}{|c|c|c|c|c|c|c|}
\hline Item & ICD-9 Description & ICD-9 Code & $\begin{array}{l}\text { Weighted } \\
\text { Frequency }\end{array}$ & $\begin{array}{c}\text { Decision } \\
\text { Round }\end{array}$ & TPS Category & $\mathrm{IoH}$ \\
\hline 1145 & Arthropathy, unspecified, lower leg & 71696 & 107644 & $\mathrm{MN}$ & Musculoskeletal & 37 \\
\hline 1146 & Malignant essential hypertension & 4010 & 100662 & $\mathrm{MN}$ & Cardiovascular & 80 \\
\hline 1147 & Other and unspecified ovarian cyst & 6202 & 188818 & $\mathrm{MN}$ & Reproductive.Female & 42 \\
\hline 1148 & Psychosexual dysfunction with inhibit. .. & 30272 & 21003 & $\mathrm{EC}$ & Psychogenic & 42 \\
\hline 1149 & Unspecified erythematous condition & 6959 & 120028 & $\mathrm{MN}$ & Integumentary & 26 \\
\hline 1150 & Other and unspecified hyperlipidemia & 2724 & 11949820 & $\mathrm{MN}$ & Cardiovascular & 47 \\
\hline 1151 & Special screening for diabetes mellitus & V771 & 37972 & $\mathrm{EC}$ & Endocrine & 45 \\
\hline 1152 & Hallux varus (acquired) & 7351 & 3415 & $\mathrm{MN}$ & Musculoskeletal & 23 \\
\hline 1153 & Enthesopathy of knee, unspecified & 72660 & 56722 & $\mathrm{MN}$ & Musculoskeletal & 34 \\
\hline 1155 & Open wound of buttock without mention. . . & 8770 & 1807 & $\mathrm{MN}$ & Integumentary & 40 \\
\hline 1157 & Abnormal liver function study & 7948 & 61560 & $\mathrm{MN}$ & Gastrointestinal & 47 \\
\hline 1158 & Renovascular unspecified secondary hy... & 40591 & 27114 & $\mathrm{EC}$ & Nephrologic & 60 \\
\hline 1159 & Basal cell carcinoma of skin, site unspecified & 17391 & 39979 & $\mathrm{MN}$ & Integumentary & 45 \\
\hline 1160 & Blister of foot and toe(s) w/o mentio... & 9172 & 20171 & $\mathrm{MN}$ & Integumentary & 20 \\
\hline 1161 & Cystic kidney disease, unspecified & 75310 & 56683 & $\mathrm{MN}$ & Nephrologic & 52 \\
\hline 1162 & Enthesopathy of specified site & 72690 & 390396 & $\mathrm{MN}$ & Musculoskeletal & 32 \\
\hline 1163 & Hemorr from threatend abortn antepart. . & 64003 & 4738 & $\mathrm{MN}$ & Reproductive.Female & 74 \\
\hline 1164 & Sarcoidosis & 135 & 18756 & $\mathrm{EC}$ & Nonspecific & 57 \\
\hline 1165 & Other constipation & 56409 & 6466 & $\mathrm{MN}$ & Gastrointestinal & 40 \\
\hline 1166 & Unspecified late effects of cerebrova... & 4389 & 133125 & $\mathrm{MN}$ & Neurologic & 55 \\
\hline 1167 & Shoulder and upper arm injury, other... & 9592 & 136983 & $\mathrm{MN}$ & Musculoskeletal & 35 \\
\hline 1168 & Hypertrophy (benign) prostate w/urina. . . & 60001 & 160866 & $\mathrm{MN}$ & Reproductive.Male & 44 \\
\hline 1169 & Diabetes w/peripheral circulatory dis... & 25070 & 28874 & $\mathrm{MN}$ & Endocrine & 57 \\
\hline 1170 & Aortic aneurysm of unspecified site w. . & 4419 & 42620 & $\mathrm{MN}$ & Cardiovascular & 65 \\
\hline 1171 & Aftercare traumatic fract of other bone & V5419 & 7519 & $\mathrm{MN}$ & Musculoskeletal & 34 \\
\hline 1172 & Due to inhalation of food or vomitus & 5070 & 6783 & $\mathrm{MN}$ & Respiratory & 55 \\
\hline 1173 & Coronary atherosclerosis of unspecifi... & 41400 & 2176488 & $\mathrm{MN}$ & Cardiovascular & 60 \\
\hline 1175 & Arteriosclerotic dementia,uncomplicated & 29040 & 28430 & $\mathrm{MN}$ & Neurologic & 55 \\
\hline 1176 & Other disorders of lipoid metabolism & 2728 & 45880 & EC & Cardiovascular & 42 \\
\hline 1177 & Unspecified symptom associated with $\mathrm{f}$. . . & 6259 & 114528 & $\mathrm{EC}$ & Reproductive.Female & 41 \\
\hline 1178 & Burn of upper limb, unspecified site,... & 94300 & 35958 & $\mathrm{MN}$ & Integumentary & 37 \\
\hline 1179 & Venous embolism and thrombosis of uns... & 4539 & 26507 & $\mathrm{EC}$ & Cardiovascular & 63 \\
\hline 1180 & Peritoneal adhesions & 5680 & 12247 & $\mathrm{MN}$ & Gastrointestinal & 47 \\
\hline 1181 & Chronic lymphoid leukemia w/o mention. . . & 20410 & 49361 & $\mathrm{MN}$ & Hematologic.Immune & 60 \\
\hline 1183 & Abrasion or friction burn of foot and... & 9170 & 58748 & $\mathrm{MN}$ & Integumentary & 24 \\
\hline 1184 & Nonorganic sleep disorder, unspecified & 30740 & 15586 & $\mathrm{MN}$ & Psychogenic & 37 \\
\hline 1185 & Atresia and stenosis of aorta & 74722 & 37630 & $\mathrm{MN}$ & Cardiovascular & 63 \\
\hline 1186 & Amyloidosis, unspecified & 27730 & 8698 & $\mathrm{MN}$ & Nonspecific & 50 \\
\hline 1187 & Blisters, epidermal loss [2nd degree]... & 94321 & 22017 & $\mathrm{MN}$ & Integumentary & 26 \\
\hline 1188 & Conduction disorder, unspecified & 4269 & 16991 & $\mathrm{EC}$ & Cardiovascular & 42 \\
\hline 1189 & Alcohol withdrawal & 29181 & 28430 & $\mathrm{EC}$ & Psychogenic & 65 \\
\hline 1190 & Long-term (current) use of other medi. . & V5869 & 305595 & $\mathrm{MN}$ & Nonspecific & 31 \\
\hline 1191 & Other unspecified back disorders & 7249 & 56453 & $\mathrm{MN}$ & Musculoskeletal & 34 \\
\hline 1192 & Diabetes w/unspecified complication, ... & 25091 & 3445 & $\mathrm{MN}$ & Endocrine & 55 \\
\hline 1194 & Other specified viral warts & 7819 & 137071 & $\mathrm{MN}$ & Integumentary & 23 \\
\hline 1195 & Cystitis, unspecified & 5959 & 214142 & $\mathrm{EC}$ & Nephrologic & 37 \\
\hline 1196 & Unspecified diseases of blood and blo... & 2899 & 14090 & $\mathrm{MN}$ & Hematologic.Immune & 41 \\
\hline 1197 & Postsurgical hypothyroidism & 2440 & 37630 & $\mathrm{MN}$ & Endocrine & 47 \\
\hline
\end{tabular}




\begin{tabular}{|c|c|c|c|c|c|c|}
\hline Item & ICD-9 Description & ICD-9 Code & $\begin{array}{l}\text { Weighted } \\
\text { Frequency }\end{array}$ & $\begin{array}{l}\text { Decision } \\
\text { Round }\end{array}$ & TPS Category & $\mathrm{IoH}$ \\
\hline 1198 & Closed other, multiple, and ill-defin... & 8270 & 16425 & $\mathrm{MN}$ & Musculoskeletal & 43 \\
\hline 1199 & Infection of genitourinary tract in p... & 64663 & 28430 & $\mathrm{EC}$ & Nephrologic & 42 \\
\hline 1200 & Patellar tendinitis & 72664 & 30528 & $\mathrm{MN}$ & Musculoskeletal & 37 \\
\hline 1201 & Other and unspecified alcohol depende. . & 30391 & 8437 & $\mathrm{MN}$ & Psychogenic & 52 \\
\hline 1202 & Exostosis of unspecified site & 72691 & 58123 & $\mathrm{EC}$ & Musculoskeletal & 26 \\
\hline 1203 & Prophylactic vaccin/inoculatn agnst $\mathrm{d}$. . . & V061 & 265375 & $\mathrm{MN}$ & Population.based.Care & 35 \\
\hline 1204 & Nuclear sclerosis & 36616 & 11463 & $\mathrm{EC}$ & Special.Sensory & 45 \\
\hline 1205 & Reflex sympathetic dystrophy of other. . & 33729 & 13439 & $\mathrm{MN}$ & Neurologic & 52 \\
\hline 1206 & Cerebral aneurysm, nonruptured & 4373 & 53473 & $\mathrm{MN}$ & Neurologic & 73 \\
\hline 1207 & Acute nonsuppurative otitis media, un. . & 38100 & 33228 & $\mathrm{MN}$ & Special.Sensory & 38 \\
\hline 1208 & Urge incontinence & 78831 & 68040 & $\mathrm{EC}$ & Nephrologic & 41 \\
\hline 1209 & Other disorders of coccyx & 72479 & 135948 & $\mathrm{MN}$ & Musculoskeletal & 26 \\
\hline 1210 & Leukocytopenia, unspecified & 28850 & 7595 & $\mathrm{MN}$ & Hematologic.Immune & 49 \\
\hline 1211 & Down`s syndrome & 7580 & 6288 & $\mathrm{MN}$ & Nonspecific & 49 \\
\hline 1212 & Other symptoms involving cardiovascul. . . & 7859 & 149751 & $\mathrm{MN}$ & Cardiovascular & 39 \\
\hline 1213 & Thyrotoxicosis without mention of goi. . & 24290 & 352344 & $\mathrm{MN}$ & Endocrine & 61 \\
\hline 1214 & Opn wound of hand except fingr(s) alo... & 8820 & 157582 & $\mathrm{MN}$ & Integumentary & 41 \\
\hline 1215 & Female infertility associated with an... & 6280 & 43369 & $\mathrm{MN}$ & Reproductive.Female & 47 \\
\hline 1216 & Other specified diseases of pulmonary... & 4178 & 5739 & $\mathrm{MN}$ & Respiratory & 47 \\
\hline 1217 & Epistaxis & 7847 & 312632 & $\mathrm{EC}$ & Respiratory & 35 \\
\hline 1218 & Abnor gland Papanicolaou smear of cer. . & 79500 & 141858 & $\mathrm{MN}$ & Reproductive.Female & 52 \\
\hline 1219 & $\begin{array}{l}\text { Pregnancy examination or test, positive } \\
\text { result }\end{array}$ & V7242 & 15464 & $\mathrm{MN}$ & Reproductive.Female & 44 \\
\hline 1220 & Feeding problems in newborn & 77931 & 12455 & $\mathrm{EC}$ & Nonspecific & 55 \\
\hline 1222 & Benign neoplasm of colon & 2113 & 127642 & $\mathrm{MN}$ & Gastrointestinal & 38 \\
\hline 1223 & Chronic lymphadenitis & 2891 & 17572 & $\mathrm{MN}$ & Hematologic.Immune & 44 \\
\hline 1224 & Mononeuritis of unspecified site & 3559 & 352035 & $\mathrm{MN}$ & Neurologic & 44 \\
\hline 1225 & Closed fracture of unspecified part o... & 81000 & 48055 & $\mathrm{MN}$ & Musculoskeletal & 44 \\
\hline 1226 & Dyspepsia and other specified disorde... & 5368 & 426647 & $\mathrm{MN}$ & Gastrointestinal & 41 \\
\hline 1227 & Acquired absence of breast & V4571 & 3298 & $\mathrm{EC}$ & Reproductive.Female & 39 \\
\hline 1228 & Unspec electiv surgery for purpos oth. . & V509 & 11414 & $\mathrm{MN}$ & Nonspecific & 34 \\
\hline 1229 & Encounter for removal of sutures & V5832 & 411691 & $\mathrm{MN}$ & Integumentary & 11 \\
\hline 1230 & Abdominal pain, epigastric & 78906 & 267975 & $\mathrm{MN}$ & Gastrointestinal & 49 \\
\hline 1231 & Orchitis and epididymitis in diseases... & 60491 & 7140 & $\mathrm{MN}$ & Reproductive.Male & 52 \\
\hline 1232 & Cerebral artery occlusion, unspec, wi... & 43491 & 353554 & $\mathrm{EC}$ & Neurologic & 80 \\
\hline 1233 & Ulcer of other part of foot, exc decubitus & 70715 & 45522 & $\mathrm{MN}$ & Integumentary & 49 \\
\hline 1234 & Swelling or mass of eye & 37992 & 46806 & $\mathrm{MN}$ & Special.Sensory & 54 \\
\hline 1235 & Lipoprotein deficiencies & 2725 & 77212 & $\mathrm{EC}$ & Cardiovascular & 39 \\
\hline 1236 & Personal history of urinary (tract) infection & V1302 & 34490 & $\mathrm{EC}$ & Nephrologic & 32 \\
\hline 1237 & Hallux valgus (acquired) & 7350 & 23728 & $\mathrm{MN}$ & Musculoskeletal & 26 \\
\hline 1238 & Other nonspecific abnormalserum enzym. . & 7905 & 10955 & $\mathrm{MN}$ & Nonspecific & 37 \\
\hline 1240 & Other seborrheic keratosis & 70219 & 319320 & $\mathrm{MN}$ & Integumentary & 26 \\
\hline 1241 & Purulent endophthalmitis, unspecified & 36000 & 78202 & $\mathrm{MN}$ & Special.Sensory & 64 \\
\hline 1242 & Prophylactic vaccinatn/inocula agnst ... & V059 & 306904 & $\mathrm{MN}$ & Population.based.Care & 35 \\
\hline 1243 & Unspecified general medical examination & V709 & 20149 & $\mathrm{EC}$ & Patient.based.Systems & 38 \\
\hline 1244 & Unspecified site of sprain and strain & 8489 & 220213 & $\mathrm{MN}$ & Musculoskeletal & 29 \\
\hline 1245 & Other specified diseases due to viruses & 7889 & 131292 & $\mathrm{MN}$ & Nonspecific & 32 \\
\hline 1246 & Osteoarthrosis, localized, primary, s. . . & 71510 & 16224 & $\mathrm{MN}$ & Musculoskeletal & 38 \\
\hline 1247 & Unspecified diseases of conjunctiva d. . . & 7799 & 17871 & $\mathrm{MN}$ & Special.Sensory & 38 \\
\hline 1248 & Hemangioma of other sites & 22809 & 11942 & $\mathrm{EC}$ & Integumentary & 32 \\
\hline
\end{tabular}




\begin{tabular}{|c|c|c|c|c|c|c|}
\hline Item & ICD-9 Description & ICD-9 Code & $\begin{array}{l}\text { Weighted } \\
\text { Frequency }\end{array}$ & $\begin{array}{l}\text { Decision } \\
\text { Round }\end{array}$ & TPS Category & $\mathrm{IoH}$ \\
\hline 1249 & Automatic implantable cardiac defibri. . . & V4502 & 3496 & $\mathrm{MN}$ & Cardiovascular & 52 \\
\hline 1250 & Conversion disorder & 30011 & 15990 & $\mathrm{MN}$ & Psychogenic & 49 \\
\hline 1251 & $\begin{array}{l}\text { Contact w/and (suspected) exposure to other } \\
\text { poten hazard chem }\end{array}$ & V872 & 28430 & $\mathrm{MN}$ & Nonspecific & 34 \\
\hline 1252 & Benign hypertensive renal disease w/o... & 40310 & 80631 & $\mathrm{MN}$ & Nephrologic & 52 \\
\hline 1253 & Ocular hypertension & 36504 & 60559 & $\mathrm{MN}$ & Special.Sensory & 58 \\
\hline 1254 & Other abnormality of red blood cells & 79009 & 28430 & $\mathrm{MN}$ & Hematologic.Immune & 44 \\
\hline 1255 & Chronic post-traumatic headache & 33922 & 12932 & $\mathrm{MN}$ & Neurologic & 49 \\
\hline 1256 & Neoplasm of uncertain behavior of tra. . . & 2357 & 11439 & $\mathrm{EC}$ & Respiratory & 57 \\
\hline 1257 & Other pulmonary embolism and infarction & 41519 & 303917 & $\mathrm{MN}$ & Respiratory & 80 \\
\hline 1258 & Other hypersomnia & 78054 & 50202 & $\mathrm{MN}$ & Neurologic & 44 \\
\hline 1259 & Diabetes w/peripheral circulatory dis... & 25072 & 34771 & $\mathrm{MN}$ & Endocrine & 60 \\
\hline 1260 & Other fluid overload & 27669 & 16002 & $\mathrm{MN}$ & Cardiovascular & 58 \\
\hline 1261 & Postnasal drip & 78491 & 212208 & $\mathrm{MN}$ & Special.Sensory & 22 \\
\hline 1262 & Bronchiectasis w/out acute exacerbation & 4940 & 108392 & $\mathrm{MN}$ & Respiratory & 41 \\
\hline 1264 & Sprains and strains of lateral collat... & 8440 & 37630 & $\mathrm{MN}$ & Musculoskeletal & 35 \\
\hline 1265 & Encounter for therapeutic drug monitor.. & V5883 & 140775 & $\mathrm{MN}$ & Nonspecific & 29 \\
\hline 1267 & Anomalies of foot, NEC & 75567 & 15541 & $\mathrm{MN}$ & Musculoskeletal & 34 \\
\hline 1268 & Observation following other accident & V714 & 12203 & $\mathrm{MN}$ & Nonspecific & 28 \\
\hline 1269 & Sprains and strains of ribs & 8483 & 28072 & $\mathrm{MN}$ & Musculoskeletal & 35 \\
\hline 1270 & Disorder of bone and cartilage, unspe... & 73390 & 818377 & $\mathrm{MN}$ & Musculoskeletal & 35 \\
\hline 1271 & Dental caries, unspecified & 52100 & 48597 & $\mathrm{EC}$ & Special.Sensory & 35 \\
\hline 1272 & Other anomalies of cervix, vagina, ex. . . & 75249 & 3591 & $\mathrm{MN}$ & Reproductive.Female & 41 \\
\hline 1273 & Cervical spondylosis without myelopathy & 7210 & 139154 & $\mathrm{MN}$ & Musculoskeletal & 41 \\
\hline 1274 & Temporomandibular joint disorders, un. .. & 52460 & 108604 & $\mathrm{MN}$ & Musculoskeletal & 38 \\
\hline 1276 & Malignant neoplasm of stomach, unspec. . . & 1519 & 28430 & $\mathrm{MN}$ & Gastrointestinal & 74 \\
\hline 1277 & Other diseases of spleen & 28959 & 7480 & $\mathrm{EC}$ & Hematologic.Immune & 44 \\
\hline 1279 & Other ill-defined conditions & 79989 & 355945 & $\mathrm{MN}$ & Nonspecific & 30 \\
\hline 1280 & Dementia with Lewy bodies & 33182 & 33988 & $\mathrm{MN}$ & Neurologic & 58 \\
\hline 1281 & Rosacea & 6953 & 298315 & $\mathrm{MN}$ & Integumentary & 29 \\
\hline 1282 & Osteoarthrosis, unspec generalized or. . . & 71594 & 8782 & $\mathrm{MN}$ & Musculoskeletal & 38 \\
\hline 1283 & Unspecified visual loss & 3699 & 39913 & $\mathrm{MN}$ & Special.Sensory & 61 \\
\hline 1284 & Pain in joint of site unspecified & 71940 & 817950 & $\mathrm{MN}$ & Musculoskeletal & 35 \\
\hline 1285 & Pain in joint of shoulder region & 71941 & 2228940 & $\mathrm{MN}$ & Musculoskeletal & 38 \\
\hline 1286 & Croup & 4644 & 54620 & $\mathrm{MN}$ & Respiratory & 47 \\
\hline 1287 & Abdominal aneurysm without mention of. . . & 4414 & 43780 & $\mathrm{MN}$ & Cardiovascular & 61 \\
\hline 1289 & Nodular prostate w/o urinary obstruction & 60010 & 12247 & $\mathrm{MN}$ & Reproductive.Male & 41 \\
\hline 1290 & Closed fracture of other metatarsal b. . & 82525 & 8184 & $\mathrm{MN}$ & Musculoskeletal & 47 \\
\hline 1291 & Other iatrogenic hypothyroidism & 2443 & 3742 & $\mathrm{MN}$ & Endocrine & 47 \\
\hline 1292 & Other disorders of menstruation & 6268 & 123842 & $\mathrm{MN}$ & Reproductive.Female & 44 \\
\hline 1293 & $\begin{array}{l}\text { Basal cell carcinoma of skin of trunk, except } \\
\text { scrotum }\end{array}$ & 17351 & 21003 & $\mathrm{MN}$ & Integumentary & 47 \\
\hline 1294 & Other specified affective psychoses & 29699 & 15980 & $\mathrm{MN}$ & Psychogenic & 58 \\
\hline 1295 & Medial epicondylitis & 72631 & 75306 & $\mathrm{MN}$ & Musculoskeletal & 35 \\
\hline 1296 & Allergic rhinitis due to other allergen & 4778 & 50947 & $\mathrm{EC}$ & Respiratory & 38 \\
\hline 1297 & Unspecified disorder of joint, pelvic. .. & 71995 & 26211 & $\mathrm{MN}$ & Musculoskeletal & 35 \\
\hline 1298 & Unspecified disorder of the teeth and. . . & 5259 & 178335 & $\mathrm{EC}$ & Special.Sensory & 29 \\
\hline 1299 & Hordeolum internum & 37312 & 36567 & $\mathrm{EC}$ & Special.Sensory & 29 \\
\hline 1300 & Heart failure, unspecified & 4289 & 30584 & $\mathrm{MN}$ & Cardiovascular & 67 \\
\hline 1301 & Long-term (current) use of anticoagul. . . & V5861 & 361576 & $\mathrm{EC}$ & Cardiovascular & 46 \\
\hline
\end{tabular}




\begin{tabular}{|c|c|c|c|c|c|c|}
\hline Item & ICD-9 Description & ICD-9 Code & $\begin{array}{l}\text { Weighted } \\
\text { Frequency }\end{array}$ & $\begin{array}{l}\text { Decision } \\
\text { Round }\end{array}$ & TPS Category & $\mathrm{IoH}$ \\
\hline 1304 & Diaper or napkin rash & 6910 & 149913 & $\mathrm{MN}$ & Integumentary & 32 \\
\hline 1305 & Benign neoplasm of stomach & 2111 & 5987 & $\mathrm{MN}$ & Gastrointestinal & 43 \\
\hline 1306 & Previous cesarean delivery, anteprtum. . . & 65423 & 23883 & $\mathrm{MN}$ & Reproductive.Female & 29 \\
\hline 1307 & Mitral valve disorders & 4240 & 88762 & $\mathrm{MN}$ & Cardiovascular & 49 \\
\hline 1308 & Other mucopurulent conjunctivitis & 37203 & 55543 & $\mathrm{MN}$ & Special.Sensory & 36 \\
\hline 1309 & Other chronic allergic conjunctivitis & 37214 & 112045 & $\mathrm{MN}$ & Special.Sensory & 36 \\
\hline 1310 & Chronic osteomyelitis of lower leg & 73016 & 17148 & $\mathrm{MN}$ & Musculoskeletal & 62 \\
\hline 1311 & Other and unspecified noninfectious $g .$. & 5589 & 844929 & $\mathrm{EC}$ & Gastrointestinal & 43 \\
\hline 1312 & Occlusion and stenosis of carotid art. . & 43310 & 77585 & $\mathrm{EC}$ & Cardiovascular & 72 \\
\hline 1313 & Degeneration of thoracic or thoracolu. . & 72251 & 33790 & $\mathrm{MN}$ & Musculoskeletal & 46 \\
\hline 1314 & Migraine, unspecified without mention. . . & 34690 & 1649174 & $\mathrm{MN}$ & Neurologic & 46 \\
\hline 1315 & Sicca syndrome & 7102 & 28430 & $\mathrm{EC}$ & Nonspecific & 43 \\
\hline 1316 & Other cellulitis and abscess of face & 6820 & 95968 & $\mathrm{MN}$ & Integumentary & 62 \\
\hline 1317 & Contact and allergic dermatitis of ey... & 37332 & 28430 & $\mathrm{MN}$ & Special.Sensory & 36 \\
\hline 1318 & Spontaneous abortion without mention ... & 63490 & 22703 & $\mathrm{MN}$ & Reproductive.Female & 56 \\
\hline 1320 & Post term pregnancy, antepartum condi. . . & 64513 & 9237 & $\mathrm{MN}$ & Reproductive.Female & 46 \\
\hline 1321 & Multiple myeloma w/o mention of remis... & 20300 & 53239 & $\mathrm{MN}$ & Hematologic.Immune & 65 \\
\hline 1323 & Unspecified disorder of metabolism & 2779 & 10943 & $\mathrm{EC}$ & Endocrine & 39 \\
\hline 1324 & Other alopecia & 70409 & 2035 & $\mathrm{MN}$ & Integumentary & 32 \\
\hline 1325 & Closed dislocation of finger, unspeci... & 83400 & 37630 & $\mathrm{MN}$ & Musculoskeletal & 36 \\
\hline 1326 & Other and unspecified capillary diseases & 4489 & 19096 & $\mathrm{MN}$ & Cardiovascular & 43 \\
\hline 1327 & Blister of hip, thigh, leg, and ankle... & 9162 & 3177 & $\mathrm{MN}$ & Integumentary & 25 \\
\hline 1329 & Chronic rhinitis & 4720 & 830352 & $\mathrm{EC}$ & Respiratory & 36 \\
\hline 1330 & Pressure ulcer stage III & 70723 & 28236 & $\mathrm{MN}$ & Integumentary & 62 \\
\hline 1331 & Neoplasm of uncertain behavior of neu. . . & 23770 & 37630 & $\mathrm{EC}$ & Neurologic & 66 \\
\hline 1332 & Other conduct disorder & 31289 & 6022 & $\mathrm{MN}$ & Psychogenic & 43 \\
\hline 1333 & Hyperparathyroidism, unspecified & 25200 & 5159 & $\mathrm{MN}$ & Endocrine & 56 \\
\hline 1334 & Idiopathic urticaria & 7081 & 8187 & $\mathrm{EC}$ & Integumentary & 39 \\
\hline 1335 & Other specified diseases of hair and ... & 7048 & 155724 & $\mathrm{MN}$ & Integumentary & 28 \\
\hline 1336 & Removal of intrauterine contraceptive device & V2512 & 20879 & $\mathrm{MN}$ & Reproductive.Female & 39 \\
\hline 1337 & Alopecia areata & 70401 & 5159 & $\mathrm{MN}$ & Integumentary & 39 \\
\hline 1338 & Endothelial corneal dystrophy & 37157 & 1672 & $\mathrm{MN}$ & Special.Sensory & 54 \\
\hline 1339 & Pain in joint of upper arm & 71942 & 162192 & $\mathrm{MN}$ & Musculoskeletal & 36 \\
\hline 1340 & Other malaise and fatigue & 78079 & 4001378 & $\mathrm{MN}$ & Nonspecific & 39 \\
\hline 1342 & Unspecified hypertrophic and atrophic. . & 7019 & 166038 & $\mathrm{EC}$ & Integumentary & 31 \\
\hline 1343 & Displacement of intervertebral disc, ... & 7222 & 198959 & $\mathrm{MN}$ & Musculoskeletal & 49 \\
\hline 1344 & External hemorrhoids without mention ... & 4553 & 7828 & $\mathrm{MN}$ & Gastrointestinal & 39 \\
\hline 1345 & $\begin{array}{l}\text { Migraine unspecif, w/o intract migr, so } \\
\text { stat. . }\end{array}$ & 34693 & 3097 & $\mathrm{MN}$ & Neurologic & 51 \\
\hline 1346 & Intervertebral disc disorder with mye... & 72273 & 5126 & $\mathrm{MN}$ & Musculoskeletal & 54 \\
\hline 1347 & Sprains and strains of lumbosacral (j... & 8460 & 111697 & $\mathrm{MN}$ & Musculoskeletal & 36 \\
\hline 1348 & Glossitis & 5290 & 60102 & $\mathrm{EC}$ & Special.Sensory & 39 \\
\hline 1349 & Other medical examination for adminis. . . & V703 & 1398641 & $\mathrm{EC}$ & Special.Sensory & 32 \\
\hline 1350 & Dermatophytosis of groin and perianal. . & 1103 & 149605 & $\mathrm{MN}$ & Integumentary & 32 \\
\hline 1351 & Achilles bursitis or tendinitis & 72671 & 64608 & $\mathrm{MN}$ & Musculoskeletal & 43 \\
\hline 1352 & Malignant neoplasm of lower limb & 1955 & 3798 & $\mathrm{MN}$ & Musculoskeletal & 72 \\
\hline 1353 & Acute, but ill-defined, cerebrovascul. . . & 436 & 16617 & $\mathrm{MN}$ & Neurologic & 76 \\
\hline 1354 & Cervicitis and endocervicitis & 6160 & 9508 & $\mathrm{MN}$ & Reproductive.Female & 52 \\
\hline
\end{tabular}




\begin{tabular}{|c|c|c|c|c|c|c|}
\hline Item & ICD-9 Description & ICD-9 Code & $\begin{array}{l}\text { Weighted } \\
\text { Frequency }\end{array}$ & $\begin{array}{c}\text { Decision } \\
\text { Round }\end{array}$ & TPS Category & $\mathrm{IoH}$ \\
\hline 1355 & Dysphagia, unspecified & 78720 & 361073 & $\mathrm{MN}$ & Gastrointestinal & 56 \\
\hline 1356 & Enthesopathy of wrist and carpus & 7264 & 53379 & $\mathrm{MN}$ & Musculoskeletal & 36 \\
\hline 1357 & Other musculoskeletal symptoms refera... & 72989 & 74239 & $\mathrm{MN}$ & Musculoskeletal & 32 \\
\hline 1358 & Acute bronchospasm & 51911 & 131077 & $\mathrm{MN}$ & Respiratory & 65 \\
\hline 1359 & Prolonged depressive reaction & 3091 & 11829 & $\mathrm{MN}$ & Psychogenic & 59 \\
\hline 1360 & Acquired deformity of pelvis & 7386 & 17707 & $\mathrm{MN}$ & Musculoskeletal & 39 \\
\hline 1361 & Transient disorder of initiating or $\mathrm{m} .$. & 30741 & 15086 & $\mathrm{EC}$ & Psychogenic & 39 \\
\hline 1362 & Mental disorders, antepartum conditio. . . & 64843 & 19958 & $\mathrm{MN}$ & Psychogenic & 52 \\
\hline 1363 & Abdominal pain, generalized & 78907 & 103259 & $\mathrm{MN}$ & Gastrointestinal & 59 \\
\hline 1364 & Infantile cerebral palsy, unspecified & 3439 & 84275 & $\mathrm{MN}$ & Neurologic & 69 \\
\hline 1365 & Other specified anomalies of spinal cord & 74259 & 28430 & $\mathrm{MN}$ & Neurologic & 56 \\
\hline 1366 & Herpes zoster with other nervous syst. . . & 5319 & 81700 & EC & Neurologic & 59 \\
\hline 1367 & Hand, except finger injury, other and. . & 9594 & 70102 & $\mathrm{MN}$ & Musculoskeletal & 41 \\
\hline 1368 & Unspecified disorder of esophagus & 5309 & 84223 & $\mathrm{MN}$ & Gastrointestinal & 43 \\
\hline 1369 & Nonsuppurative otitis media, not spec. . . & 3814 & 307151 & $\mathrm{MN}$ & Special.Sensory & 43 \\
\hline 1370 & Acute myeloid leukemia w/o mention of. . . & 20500 & 5268 & $\mathrm{MN}$ & Hematologic.Immune & 80 \\
\hline 1371 & $\begin{array}{l}\text { Basal cell carcinoma skin of other and } \\
\text { unspec parts of face }\end{array}$ & 17331 & 3591 & $\mathrm{MN}$ & Integumentary & 45 \\
\hline 1372 & Unspecified delay in development & 3159 & 29187 & $\mathrm{EC}$ & Nonspecific & 62 \\
\hline 1373 & Carbuncle and furuncle of buttock & 6805 & 26999 & $\mathrm{MN}$ & Integumentary & 46 \\
\hline 1374 & Poisoning by unspecified drug or medi. . . & 9779 & 4419 & $\mathrm{MN}$ & Nonspecific & 76 \\
\hline 1375 & Dermatographic urticaria & 7083 & 5916 & $\mathrm{EC}$ & Integumentary & 36 \\
\hline 1376 & Insect bite of face/neck/scalp,nonven. .. & 9104 & 20341 & $\mathrm{MN}$ & Integumentary & 21 \\
\hline 1377 & Bell’s palsy & 3510 & 122110 & $\mathrm{MN}$ & Neurologic & 59 \\
\hline 1378 & Asthma, unspecified with acute exacerbat & 49392 & 237951 & $\mathrm{MN}$ & Respiratory & 62 \\
\hline 1379 & Unspecified outcome of delivery & V279 & 15464 & $\mathrm{MN}$ & Reproductive.Female & 34 \\
\hline 1380 & Rash and other nonspecific skin eruption & 7821 & 1487116 & $\mathrm{MN}$ & Integumentary & 35 \\
\hline 1381 & Other complications due to renal dial... & 99673 & 24215 & $\mathrm{MN}$ & Nephrologic & 59 \\
\hline 1382 & Extrinsic asthma with acute exacerbation & 49302 & 51268 & $\mathrm{MN}$ & Respiratory & 76 \\
\hline 1383 & Supervision of other normal pregnancy & V221 & 1442891 & $\mathrm{MN}$ & Reproductive.Female & 43 \\
\hline 1384 & Viremia, unspecified & 7908 & 14219 & $\mathrm{MN}$ & Nonspecific & 46 \\
\hline 1386 & Ulcer of anus and rectum & 56941 & 37630 & $\mathrm{MN}$ & Gastrointestinal & 52 \\
\hline 1387 & Other peripheral enthesopathies & 7268 & 15749 & $\mathrm{MN}$ & Musculoskeletal & 39 \\
\hline 1388 & Chronic/unspec gastric ulcer w/perfor. . . & 53150 & 27114 & $\mathrm{MN}$ & Gastrointestinal & 65 \\
\hline 1389 & Acute swimmers`ear & 38012 & 12289 & $\mathrm{MN}$ & Special.Sensory & 43 \\
\hline 1390 & Torsion of testis, unspecified & 60820 & 32767 & $\mathrm{MN}$ & Reproductive.Male & 86 \\
\hline 1392 & Pure hypercholesterolemia & 2720 & 4633304 & $\mathrm{MN}$ & Cardiovascular & 43 \\
\hline 1393 & Cellulitis and abscess of the oral so... & 5283 & 17594 & EC & Special.Sensory & 49 \\
\hline 1394 & Scar conditions and fibrosis of skin & 7092 & 6288 & $\mathrm{MN}$ & Integumentary & 32 \\
\hline 1395 & Other specified disorders of skin & 7098 & 7828 & $\mathrm{MN}$ & Integumentary & 40 \\
\hline 1396 & Major depressive disorder, recurrent ... & 29630 & 255488 & $\mathrm{MN}$ & Psychogenic & 59 \\
\hline 1397 & Acquired hypertrophic pyloric stenosis & 5370 & 15221 & $\mathrm{MN}$ & Gastrointestinal & 72 \\
\hline 1398 & Special screening for depression & V790 & 46806 & $\mathrm{MN}$ & Psychogenic & 39 \\
\hline 1399 & Celiac disease & 5790 & 47867 & $\mathrm{MN}$ & Gastrointestinal & 56 \\
\hline 1400 & Polyuria & 78842 & 123013 & $\mathrm{EC}$ & Nephrologic & 52 \\
\hline 1401 & Other diseases of respiratory system,... & 5198 & 258630 & $\mathrm{MN}$ & Respiratory & 49 \\
\hline 1402 & Other and unspecified alcohol depende. . . & 30393 & 28430 & $\mathrm{MN}$ & Psychogenic & 63 \\
\hline 1403 & Hallucinations & 7801 & 18572 & $\mathrm{MN}$ & Psychogenic & 70 \\
\hline 1404 & Acquired spondylolisthesis & 7384 & 3591 & $\mathrm{MN}$ & Musculoskeletal & 47 \\
\hline 1405 & Unspecified vitamin $\mathrm{D}$ deficiency & 2689 & 1313858 & $\mathrm{MN}$ & Endocrine & 43 \\
\hline 1408 & Unspecified nasal polyp & 4719 & 27114 & $\mathrm{MN}$ & Special.Sensory & 30 \\
\hline
\end{tabular}




\begin{tabular}{|c|c|c|c|c|c|c|}
\hline Item & ICD-9 Description & ICD-9 Code & $\begin{array}{l}\text { Weighted } \\
\text { Frequency }\end{array}$ & $\begin{array}{c}\text { Decision } \\
\text { Round }\end{array}$ & TPS Category & IoH \\
\hline 1409 & Other and unspecified nonspecific imm... & 79579 & 27114 & $\mathrm{MN}$ & Hematologic.Immune & 43 \\
\hline 1410 & Other diseases of lung, not elsewhere... & 51889 & 60346 & $\mathrm{MN}$ & Respiratory & 44 \\
\hline 1411 & Ostium secundum type atrial septal de... & 7455 & 12572 & $\mathrm{MN}$ & Cardiovascular & 63 \\
\hline 1412 & Body Mass Index 27.0 to 27.9 , adult & V8523 & 17572 & $\mathrm{EC}$ & Nonspecific & 40 \\
\hline 1413 & Other symptoms involving abdomen and ... & 7899 & 45711 & $\mathrm{MN}$ & Gastrointestinal & 37 \\
\hline 1414 & Opioid type dependence, unspecified & 30400 & 1384256 & $\mathrm{MN}$ & Psychogenic & 66 \\
\hline 1415 & Renal failure, unspecified & 586 & 100610 & $\mathrm{MN}$ & Nephrologic & 73 \\
\hline 1416 & Osteoarthrosis, unspec generalized or. . & 71593 & 28430 & $\mathrm{MN}$ & Musculoskeletal & 40 \\
\hline 1417 & Loss of height & 78191 & 28430 & $\mathrm{MN}$ & Musculoskeletal & 43 \\
\hline 1418 & Acute mucoid otitis media & 38102 & 3953 & $\mathrm{MN}$ & Special.Sensory & 43 \\
\hline 1419 & Acute cholecystitis & 5750 & 17594 & $\mathrm{MN}$ & Gastrointestinal & 73 \\
\hline 1420 & Alzheimer`s disease & 3310 & 505503 & $\mathrm{MN}$ & Neurologic & 60 \\
\hline 1421 & Neoplasms of unspecified nature bone,... & 2392 & 56957 & $\mathrm{MN}$ & Musculoskeletal & 66 \\
\hline 1422 & Systemic inflamm resp synd due to inf. . & 99591 & 37630 & $\mathrm{MN}$ & Nonspecific & 87 \\
\hline 1423 & Prophylactic vaccination/inoculation ... & V054 & 17572 & $\mathrm{MN}$ & Population.based.Care & 37 \\
\hline 1424 & Pathologic fracture of humerus & 73311 & 21003 & $\mathrm{MN}$ & Musculoskeletal & 66 \\
\hline 1425 & Unspecified disease of white blood cells & 2889 & 28430 & $\mathrm{MN}$ & Hematologic.Immune & 50 \\
\hline 1426 & Finger injury, other and unspecified & 9595 & 46806 & $\mathrm{MN}$ & Musculoskeletal & 37 \\
\hline 1427 & Unspecified essential hypertension & 4019 & 25307174 & $\mathrm{MN}$ & Cardiovascular & 47 \\
\hline 1428 & Sprains and strains of unspecified si... & 84500 & 382832 & $\mathrm{MN}$ & Musculoskeletal & 33 \\
\hline 1429 & Barrett`s esophagus & 53085 & 4487 & $\mathrm{MN}$ & Gastrointestinal & 63 \\
\hline 1430 & Other symptoms referable to joint of ... & 71963 & 11812 & $\mathrm{MN}$ & Musculoskeletal & 37 \\
\hline 1431 & Other spec complicatns of preg, antep... & 64683 & 28123 & $\mathrm{MN}$ & Reproductive.Female & 50 \\
\hline 1432 & Diffuse cystic mastopathy & 6101 & 101312 & $\mathrm{MN}$ & Reproductive.Female & 43 \\
\hline 1433 & Iron deficiency anemia, unspecified & 2809 & 280701 & $\mathrm{MN}$ & Hematologic.Immune & 50 \\
\hline 1434 & Sunburn of second degree & 69276 & 4811 & $\mathrm{MN}$ & Integumentary & 30 \\
\hline 1435 & Peripheral vascular disease, unspecified & 4439 & 420691 & $\mathrm{MN}$ & Cardiovascular & 53 \\
\hline 1436 & Other symptoms referable to joint of ... & 71961 & 17594 & $\mathrm{MN}$ & Musculoskeletal & 33 \\
\hline 1437 & Unspecified closed fracture of ankle & 8248 & 142345 & $\mathrm{MN}$ & Musculoskeletal & 50 \\
\hline 1438 & Unspecified site of spinal cord injury & 9529 & 3051 & $\mathrm{MN}$ & Neurologic & 81 \\
\hline 1439 & Arteritis, unspecified & 4476 & 41092 & $\mathrm{EC}$ & Cardiovascular & 63 \\
\hline 1440 & Other cellulitis and abscess of unspe... & 6829 & 1058575 & $\mathrm{MN}$ & Integumentary & 50 \\
\hline 1441 & Alcohol abuse, unspecified & 30500 & 189697 & $\mathrm{MN}$ & Psychogenic & 60 \\
\hline 1442 & Paraplegia & 3441 & 14058 & $\mathrm{MN}$ & Neurologic & 73 \\
\hline 1443 & Sacroiliitis, not elsewhere classified & 7202 & 176355 & $\mathrm{MN}$ & Musculoskeletal & 40 \\
\hline 1444 & Asymptomatic bacteriuria in preg ante... & 64653 & 27250 & $\mathrm{MN}$ & Reproductive.Female & 48 \\
\hline 1445 & Other specified erythematous conditions & 69589 & 74768 & $\mathrm{MN}$ & Integumentary & 26 \\
\hline 1447 & Other tear of cartilage or meniscus o... & 8362 & 33059 & $\mathrm{MN}$ & Musculoskeletal & 43 \\
\hline 1448 & Other physical therapy & V571 & 3535 & $\mathrm{MN}$ & Musculoskeletal & 32 \\
\hline 1449 & Acute nasopharyngitis [common cold] & 460 & 424632 & $\mathrm{EC}$ & Respiratory & 30 \\
\hline 1450 & General nonconvulsv epilepsy w/o ment. . . & 34500 & 7631 & $\mathrm{MN}$ & Neurologic & 63 \\
\hline 1451 & Dyskinesia of esophagus & 5305 & 53429 & $\mathrm{MN}$ & Gastrointestinal & 50 \\
\hline 1452 & Mild or unspec pre-eclampsia w/antepa... & 64243 & 7631 & $\mathrm{MN}$ & Reproductive.Female & 73 \\
\hline 1453 & Systolic heart failure, acute on chronic & 42823 & 11463 & $\mathrm{MN}$ & Cardiovascular & 87 \\
\hline 1454 & Closed fracture of unspecified bone(s. . . & 82520 & 15749 & $\mathrm{MN}$ & Musculoskeletal & 53 \\
\hline 1455 & Acute conjunctivitis, unspecified & 37200 & 88466 & $\mathrm{MN}$ & Special.Sensory & 33 \\
\hline 1456 & Frontal acute sinusitis & 4611 & 66006 & $\mathrm{EC}$ & Respiratory & 43 \\
\hline 1457 & Unspecified degree burn of thigh [any. . & 94506 & 37630 & $\mathrm{MN}$ & Integumentary & 47 \\
\hline 1458 & Polyneuropathy in diabetes & 3572 & 214605 & $\mathrm{MN}$ & Neurologic & 56 \\
\hline
\end{tabular}




\begin{tabular}{|c|c|c|c|c|c|c|}
\hline Item & ICD-9 Description & ICD-9 Code & $\begin{array}{l}\text { Weighted } \\
\text { Frequency }\end{array}$ & $\begin{array}{l}\text { Decision } \\
\text { Round }\end{array}$ & TPS Category & $\mathrm{IoH}$ \\
\hline 1459 & Other specified disorders of adrenal ... & 2558 & 29838 & $\mathrm{MN}$ & Endocrine & 56 \\
\hline 1460 & Neuralgia, neuritis, and radiculitis,... & 7292 & 267877 & $\mathrm{MN}$ & Neurologic & 50 \\
\hline 1461 & Unspecified contraceptive management & V259 & 423656 & $\mathrm{MN}$ & Reproductive.Female & 43 \\
\hline 1462 & Failure to thrive in newborn & 77934 & 13304 & $\mathrm{MN}$ & Nonspecific & 81 \\
\hline 1463 & Colostomy & V443 & 9554 & $\mathrm{MN}$ & Gastrointestinal & 47 \\
\hline 1464 & Bacteremia & 7907 & 1830 & $\mathrm{EC}$ & Hematologic.Immune & 70 \\
\hline 1465 & Other developmental speech or languag. . . & 31539 & 29644 & $\mathrm{MN}$ & Neurologic & 60 \\
\hline 1466 & Breast screening, unspecified & V7610 & 144283 & $\mathrm{MN}$ & Reproductive.Female & 33 \\
\hline 1467 & Acute suppurative otitis media w/out ... & 38200 & 329856 & $\mathrm{MN}$ & Special.Sensory & 40 \\
\hline 1468 & Mixed incontinence (female) (male) & 78833 & 1522 & $\mathrm{EC}$ & Nephrologic & 37 \\
\hline 1469 & Routine or ritual circumcision & V502 & 42629 & $\mathrm{EC}$ & Reproductive.Male & 28 \\
\hline 1472 & Orthostatic hypotension & 4580 & 28686 & $\mathrm{MN}$ & Cardiovascular & 56 \\
\hline 1474 & Neurogenic bladder NOS & 59654 & 27114 & EC & Nephrologic & 50 \\
\hline 1475 & Other viral conjunctivitis due to vir... & 778 & 27114 & $\mathrm{MN}$ & Special.Sensory & 30 \\
\hline 1476 & Oth elective surgery for purpos oth $\mathrm{t} . \mathrm{.}$ & V508 & 622784 & $\mathrm{MN}$ & Nonspecific & 43 \\
\hline 1477 & Bipolar affective disorder, depressed. . . & 29652 & 4382 & $\mathrm{MN}$ & Psychogenic & 70 \\
\hline 1478 & Person feigning illness & V652 & 37630 & $\mathrm{MN}$ & Psychogenic & 53 \\
\hline 1479 & Irritability & 79922 & 17677 & $\mathrm{EC}$ & Psychogenic & 37 \\
\hline 1480 & Other diseases of larynx, not elsewhe... & 47879 & 43115 & $\mathrm{EC}$ & Respiratory & 37 \\
\hline 1481 & Blister/epiderm loss [2nd degree] bur. . & 94421 & 23065 & $\mathrm{MN}$ & Integumentary & 43 \\
\hline 1482 & Diarrhea of presumed infectious origin & 93 & 7818 & $\mathrm{MN}$ & Gastrointestinal & 43 \\
\hline 1483 & Carpal tunnel syndrome & 3540 & 372451 & $\mathrm{MN}$ & Musculoskeletal & 37 \\
\hline 1484 & Cellulitis and abscess of finger, uns... & 68100 & 40258 & $\mathrm{MN}$ & Integumentary & 43 \\
\hline 1485 & Menstrual migraine, without mention of.. & 34640 & 11829 & $\mathrm{MN}$ & Neurologic & 40 \\
\hline 1486 & Mitral stenosis & 3940 & 3742 & $\mathrm{MN}$ & Cardiovascular & 63 \\
\hline 1487 & Hirsutism & 7041 & 19549 & $\mathrm{MN}$ & Endocrine & 43 \\
\hline 1488 & Other specified periodontal diseases & 5238 & 11829 & $\mathrm{EC}$ & Special.Sensory & 40 \\
\hline 1489 & Hyposmolality and/or hyponatremia & 2761 & 114085 & $\mathrm{EC}$ & Nonspecific & 70 \\
\hline 1490 & Open wound of elbow, without mention ... & 88101 & 8187 & $\mathrm{MN}$ & Integumentary & 47 \\
\hline 1492 & Special screening for other and unspe... & V814 & 93612 & $\mathrm{EC}$ & Respiratory & 21 \\
\hline 1493 & Palpitations & 7851 & 581937 & $\mathrm{MN}$ & Cardiovascular & 56 \\
\hline 1494 & Allergic rhinitis due to pollen & 4770 & 72010 & $\mathrm{EC}$ & Respiratory & 33 \\
\hline 1496 & Hemiplegia, unspecified, affecting un. . & 34290 & 6022 & $\mathrm{MN}$ & Neurologic & 70 \\
\hline 1497 & Intrinsic asthma without mention of s. . & 49310 & 55015 & $\mathrm{MN}$ & Respiratory & 60 \\
\hline 1499 & Seborrheic infantile dermatitis & 69012 & 27114 & $\mathrm{MN}$ & Integumentary & 33 \\
\hline 1500 & Reading disorder, unspecified & 31500 & 28430 & $\mathrm{EC}$ & Psychogenic & 47 \\
\hline 1501 & Otorrhea, unspecified & 38860 & 19177 & $\mathrm{MN}$ & Special.Sensory & 42 \\
\hline 1502 & Throat pain & 7841 & 61582 & $\mathrm{EC}$ & Respiratory & 36 \\
\hline 1503 & Pneumonia, organism unspecified & 486 & 814888 & $\mathrm{MN}$ & Respiratory & 68 \\
\hline 1505 & Closed fracture of unspecified part o... & 81220 & 72160 & $\mathrm{MN}$ & Musculoskeletal & 53 \\
\hline 1506 & Other specified complications due to ... & 5479 & 28430 & $\mathrm{MN}$ & Nonspecific & 37 \\
\hline 1507 & Neoplasm of uncertain behavior of sit. . . & 2389 & 80401 & $\mathrm{MN}$ & Nonspecific & 63 \\
\hline 1508 & Chronic hepatitis $\mathrm{C}$ without mention o... & 7054 & 37630 & $\mathrm{MN}$ & Gastrointestinal & 65 \\
\hline 1509 & Abnormal loss of weight & 78321 & 633643 & $\mathrm{MN}$ & Nonspecific & 68 \\
\hline 1510 & Unspecified disorders of menstruation & 6269 & 112235 & $\mathrm{MN}$ & Reproductive.Female & 45 \\
\hline 1511 & Major depressive disorder, recurrent ... & 29632 & 139869 & $\mathrm{MN}$ & Psychogenic & 56 \\
\hline 1512 & Other lymphomas, unspec site, extrano... & 20280 & 94967 & $\mathrm{MN}$ & Hematologic.Immune & 71 \\
\hline 1513 & Obstruction of nasolacrimal duct, neo... & 37555 & 3496 & $\mathrm{MN}$ & Special.Sensory & 37 \\
\hline 1514 & Allergic urticaria & 7080 & 46806 & $\mathrm{EC}$ & Integumentary & 42 \\
\hline 1515 & Allergy, unspecified & 9953 & 867426 & $\mathrm{MN}$ & Hematologic.Immune & 30 \\
\hline
\end{tabular}




\begin{tabular}{|c|c|c|c|c|c|c|}
\hline Item & ICD-9 Description & ICD-9 Code & $\begin{array}{l}\text { Weighted } \\
\text { Frequency }\end{array}$ & $\begin{array}{l}\text { Decision } \\
\text { Round }\end{array}$ & TPS Category & $\mathrm{IoH}$ \\
\hline 1516 & Cyst and pseudocyst of pancreas & 5772 & 17148 & $\mathrm{MN}$ & Gastrointestinal & 59 \\
\hline 1517 & Osteoarthrosis, generalized, site uns... & 71500 & 39152 & $\mathrm{MN}$ & Musculoskeletal & 39 \\
\hline 1518 & Unspecified inflammatory polyarthropathy & 7149 & 17594 & $\mathrm{MN}$ & Musculoskeletal & 53 \\
\hline 1519 & Unspecified degree burn of foot & 94502 & 16460 & $\mathrm{MN}$ & Integumentary & 48 \\
\hline 1520 & Primary focal hyperhidrosis & 70521 & 31811 & $\mathrm{MN}$ & Integumentary & 31 \\
\hline 1522 & Sialoadenitis of the salivary glands & 5272 & 3314 & EC & Special.Sensory & 42 \\
\hline 1523 & Other ovarian failure & 25639 & 40928 & $\mathrm{MN}$ & Reproductive.Female & 53 \\
\hline 1524 & Superficial injury of cornea & 9181 & 12590 & $\mathrm{MN}$ & Special.Sensory & 46 \\
\hline 1525 & Undiagnosed cardiac murmurs & 7852 & 115385 & $\mathrm{MN}$ & Cardiovascular & 53 \\
\hline 1526 & Body Mass Index 32.0 to 32.9 , adult & V8532 & 14370 & $\mathrm{EC}$ & Nonspecific & 49 \\
\hline 1527 & Maxillary chronic sinusitis & 4730 & 123044 & $\mathrm{MN}$ & Special.Sensory & 47 \\
\hline 1528 & Unspecified degree burn of hand, unsp. . . & 94400 & 4041 & $\mathrm{MN}$ & Integumentary & 47 \\
\hline 1529 & Reflex sympathetic dystrophy, unspeci. . . & 33720 & 27114 & $\mathrm{MN}$ & Neurologic & 53 \\
\hline 1530 & Unspecified hypertensive renal diseas. . & 40391 & 36620 & $\mathrm{MN}$ & Nephrologic & 68 \\
\hline 1531 & $\begin{array}{l}\text { Other nonspecific abnormal finding of lung } \\
\text { field }\end{array}$ & 79319 & 33487 & $\mathrm{MN}$ & Respiratory & 47 \\
\hline 1532 & Other and unspecified alcohol depende... & 30390 & 165538 & $\mathrm{MN}$ & Psychogenic & 59 \\
\hline 1533 & Other vitamin B12 deficiency anemia & 2811 & 23791 & EC & Hematologic.Immune & 48 \\
\hline 1534 & Other joint derangement of lower leg & 71886 & 22598 & $\mathrm{MN}$ & Musculoskeletal & 36 \\
\hline 1535 & Scrotal varices & 4564 & 10426 & $\mathrm{MN}$ & Reproductive.Male & 23 \\
\hline 1536 & Chronic respiratory failure & 51883 & 26683 & $\mathrm{MN}$ & Respiratory & 71 \\
\hline 1537 & Premature menopause & 25631 & 3460 & $\mathrm{MN}$ & Reproductive.Female & 53 \\
\hline 1538 & Atrophy of breast & 6114 & 8137 & $\mathrm{EC}$ & Reproductive.Female & 27 \\
\hline 1539 & Impetigo & 684 & 101228 & $\mathrm{MN}$ & Integumentary & 39 \\
\hline 1540 & Chronic tonsillitis & 47400 & 3458 & $\mathrm{EC}$ & Respiratory & 45 \\
\hline 1541 & Heat exhaustion, unspecified & 9925 & 11518 & $\mathrm{EC}$ & Nonspecific & 68 \\
\hline 1542 & Endocarditis in diseases classified e... & 42491 & 4382 & $\mathrm{MN}$ & Cardiovascular & 81 \\
\hline 1543 & Hypertrophy of breast & 6111 & 34416 & $\mathrm{EC}$ & Reproductive.Female & 39 \\
\hline 1544 & Other specified diseases of nail & 7038 & 3244 & $\mathrm{MN}$ & Integumentary & 13 \\
\hline 1545 & Urgency of urination & 78863 & 34718 & $\mathrm{EC}$ & Nephrologic & 45 \\
\hline 1546 & Osteoporosis, unspecified & 73300 & 967590 & $\mathrm{EC}$ & Musculoskeletal & 49 \\
\hline 1547 & Venous (peripheral) insufficiency, un. . . & 45981 & 129520 & $\mathrm{MN}$ & Cardiovascular & 48 \\
\hline 1548 & Cerebral degeneration, unspecified & 3319 & 7043 & $\mathrm{MN}$ & Neurologic & 53 \\
\hline 1549 & Prophylactic vaccin/inocu agnst typho. . & V031 & 11414 & $\mathrm{MN}$ & Population.based.Care & 26 \\
\hline 1550 & Stress incontinence, female & 6256 & 61922 & $\mathrm{EC}$ & Nephrologic & 39 \\
\hline 1551 & Unspecified fetal and neonatal jaundice & 7746 & 99277 & $\mathrm{MN}$ & Gastrointestinal & 59 \\
\hline 1552 & Diarrhea & 78791 & 990642 & $\mathrm{MN}$ & Gastrointestinal & 45 \\
\hline 1553 & Social phobia & 30023 & 8187 & $\mathrm{MN}$ & Psychogenic & 45 \\
\hline 1554 & Hypopotassemia & 2768 & 313520 & $\mathrm{EC}$ & Nonspecific & 65 \\
\hline 1555 & Tension type headache, unspecified & 33910 & 21691 & $\mathrm{MN}$ & Neurologic & 39 \\
\hline 1556 & Failure to thrive & 78341 & 48777 & $\mathrm{MN}$ & Nonspecific & 68 \\
\hline 1557 & Bronchitis, not specified as acute or... & 490 & 2994117 & $\mathrm{MN}$ & Respiratory & 42 \\
\hline 1558 & Nonspecific low blood pressure reading & 7963 & 16127 & $\mathrm{MN}$ & Cardiovascular & 48 \\
\hline 1559 & Supraventricular premature beats & 42761 & 37630 & $\mathrm{MN}$ & Cardiovascular & 51 \\
\hline 1560 & Contusion of hand(s) & 92320 & 66649 & $\mathrm{MN}$ & Musculoskeletal & 23 \\
\hline 1561 & Other acute sinusitis & 4618 & 33484 & $\mathrm{MN}$ & Special.Sensory & 42 \\
\hline 1562 & Osteoarthrosis, localized, primary, s. . & 71511 & 13706 & $\mathrm{MN}$ & Musculoskeletal & 39 \\
\hline 1563 & Chronic/unspec gastrojejunal ulcer w/. . & 53460 & 3460 & $\mathrm{MN}$ & Gastrointestinal & 59 \\
\hline 1564 & Mild hyperemesis gravidarum antepartu. . . & 64303 & 29577 & $\mathrm{MN}$ & Reproductive.Female & 42 \\
\hline
\end{tabular}




\begin{tabular}{|c|c|c|c|c|c|c|}
\hline Item & ICD-9 Description & ICD-9 Code & $\begin{array}{l}\text { Weighted } \\
\text { Frequency }\end{array}$ & $\begin{array}{l}\text { Decision } \\
\text { Round }\end{array}$ & TPS Category & $\mathrm{IoH}$ \\
\hline 1565 & Autoimmune hemolytic anemias & 2830 & 8782 & $\mathrm{MN}$ & Hematologic.Immune & 65 \\
\hline 1566 & Nervousness & 79921 & 1232 & $\mathrm{MN}$ & Psychogenic & 36 \\
\hline 1567 & Other specified acquired hypothyroidism & 2448 & 55350 & $\mathrm{MN}$ & Endocrine & 45 \\
\hline 1568 & Excessive fetal growth, antepartum co... & 65663 & 5739 & $\mathrm{EC}$ & Nonspecific & 53 \\
\hline 1569 & Other specified disorders of kidney a... & 59389 & 16002 & $\mathrm{MN}$ & Nephrologic & 50 \\
\hline 1570 & Effusion of joint of lower leg & 71906 & 128572 & $\mathrm{MN}$ & Musculoskeletal & 45 \\
\hline 1571 & Blood in stool & 5781 & 114391 & $\mathrm{MN}$ & Gastrointestinal & 56 \\
\hline 1572 & Simple chronic bronchitis & 4910 & 19549 & $\mathrm{MN}$ & Respiratory & 39 \\
\hline 1573 & Unspecified hypertensive heart diseas. . . & 40290 & 40951 & $\mathrm{MN}$ & Cardiovascular & 65 \\
\hline 1574 & Sprains and strains of unspecified si... & 8469 & 44939 & $\mathrm{MN}$ & Musculoskeletal & 30 \\
\hline 1575 & Stiffness of joint of other spec site... & 71958 & 12393 & $\mathrm{MN}$ & Musculoskeletal & 33 \\
\hline 1576 & Postmenopausal bleeding & 6271 & 45333 & $\mathrm{MN}$ & Reproductive.Female & 65 \\
\hline 1577 & Diabetes with renal manifestations, t... & 25040 & 406649 & $\mathrm{MN}$ & Endocrine & 59 \\
\hline 1578 & Dermatophytosis of the body & 1105 & 78654 & $\mathrm{MN}$ & Integumentary & 30 \\
\hline 1579 & Other specified visual disturbances & 3688 & 37630 & $\mathrm{MN}$ & Special.Sensory & 53 \\
\hline 1580 & Abdominal pain, right upper quadrant & 78901 & 314076 & $\mathrm{MN}$ & Gastrointestinal & 62 \\
\hline 1581 & Open wound of hip and thigh, without ... & 8900 & 18439 & $\mathrm{MN}$ & Integumentary & 51 \\
\hline 1582 & Unspecified degree burn of leg, unspe... & 94500 & 9934 & $\mathrm{MN}$ & Integumentary & 45 \\
\hline 1583 & Prepatellar bursitis & 72665 & 67047 & $\mathrm{MN}$ & Musculoskeletal & 39 \\
\hline 1584 & Bacterial infection, unspecified & 419 & 29743 & $\mathrm{MN}$ & Nonspecific & 59 \\
\hline 1585 & Chronic pancreatitis & 5771 & 9344 & $\mathrm{MN}$ & Gastrointestinal & 62 \\
\hline 1586 & Other disorders of lactation, postpar... & 67684 & 14370 & $\mathrm{MN}$ & Reproductive.Female & 42 \\
\hline 1587 & Foreign body of unspecified site of eye & 9309 & 24376 & $\mathrm{MN}$ & Special.Sensory & 59 \\
\hline 1588 & Neoplasm of uncertain behavior of adr. . & 2372 & 7437 & $\mathrm{MN}$ & Endocrine & 65 \\
\hline 1589 & Alcoholic cirrhosis of liver & 5712 & 19857 & $\mathrm{MN}$ & Gastrointestinal & 68 \\
\hline 1590 & Acute sinusitis, unspecified & 4619 & 2647725 & $\mathrm{MN}$ & Special.Sensory & 48 \\
\hline 1591 & Endometriosis, site unspecified & 6179 & 32716 & $\mathrm{MN}$ & Reproductive.Female & 53 \\
\hline 1592 & Impaired glucose tolerance test (oral) & 79022 & 9970 & $\mathrm{MN}$ & Endocrine & 45 \\
\hline 1593 & Major depressive disorder, recurrent ... & 29631 & 27114 & $\mathrm{MN}$ & Psychogenic & 59 \\
\hline 1594 & Personal history of surgery to other organs & V1529 & 4574 & $\mathrm{MN}$ & Nonspecific & 36 \\
\hline 1595 & Chronic motor tic disorder & 30722 & 3076 & $\mathrm{MN}$ & Neurologic & 36 \\
\hline 1596 & Other abnormal heart sounds & 7853 & 15980 & $\mathrm{MN}$ & Cardiovascular & 39 \\
\hline 1597 & Sprains and strains of other area of ... & 84509 & 4694 & $\mathrm{MN}$ & Musculoskeletal & 30 \\
\hline 1598 & Hemiplegia affecting unspecified side & 43820 & 37630 & $\mathrm{MN}$ & Neurologic & 71 \\
\hline 1599 & Obstructive chronic bronchitis w/exacer & 49121 & 266279 & $\mathrm{MN}$ & Respiratory & 74 \\
\hline 1600 & Personal history of other disorders o... & V1309 & 28943 & $\mathrm{EC}$ & Nephrologic & 33 \\
\hline 1601 & $\begin{array}{l}\text { Pregnancy examination or test, preg } \\
\text { unconfirmed }\end{array}$ & V7240 & 34248 & $\mathrm{MN}$ & Reproductive.Female & 45 \\
\hline 1602 & Intrauterine synechiae & 6215 & 7828 & $\mathrm{MN}$ & Reproductive.Female & 51 \\
\hline 1603 & Knee replacement & V4365 & 73440 & $\mathrm{MN}$ & Musculoskeletal & 50 \\
\hline 1604 & Tic disorder, unspecified & 30720 & 30196 & $\mathrm{MN}$ & Neurologic & 42 \\
\hline 1605 & Special screening for malignant neopl. . . & V762 & 422307 & $\mathrm{EC}$ & Reproductive.Female & 62 \\
\hline 1606 & Allergic purpura & 2870 & 36951 & $\mathrm{EC}$ & Integumentary & 47 \\
\hline 1607 & Other late effects of cerebrovascular... & 43889 & 7828 & $\mathrm{MN}$ & Neurologic & 51 \\
\hline 1608 & $\begin{array}{l}\text { Health supervision for newborn under } 8 \\
\text { days old }\end{array}$ & V2031 & 90108 & $\mathrm{EC}$ & Patient.based.Systems & 47 \\
\hline 1609 & Dislocation of patella, closed & 8363 & 7831 & $\mathrm{MN}$ & Musculoskeletal & 47 \\
\hline 1610 & Other osteoporosis & 73309 & 5603 & $\mathrm{EC}$ & Musculoskeletal & 51 \\
\hline 1611 & Other disorders of plasma protein met. . . & 2738 & 17594 & $\mathrm{MN}$ & Hematologic.Immune & 45 \\
\hline
\end{tabular}




\begin{tabular}{|c|c|c|c|c|c|c|}
\hline Item & ICD-9 Description & ICD-9 Code & $\begin{array}{l}\text { Weighted } \\
\text { Frequency }\end{array}$ & $\begin{array}{c}\text { Decision } \\
\text { Round }\end{array}$ & TPS Category & $\mathrm{IoH}$ \\
\hline 1613 & Hypertrophy of adenoids alone & 47412 & 13084 & $\mathrm{EC}$ & Respiratory & 22 \\
\hline 1614 & Clos fracture of sacrum and coccyx, w... & 8056 & 49773 & $\mathrm{MN}$ & Musculoskeletal & 47 \\
\hline 1616 & Unspecified disease of nail & 7039 & 3953 & $\mathrm{MN}$ & Integumentary & 15 \\
\hline 1617 & Hypocalcemia & 27541 & 63869 & $\mathrm{MN}$ & Endocrine & 51 \\
\hline 1618 & Jaw pain & 78492 & 84834 & $\mathrm{MN}$ & Musculoskeletal & 47 \\
\hline 1620 & Unspecified glaucoma & 3659 & 188833 & $\mathrm{MN}$ & Special.Sensory & 51 \\
\hline 1621 & Dermatitis due to drugs and medicines & 6930 & 9606 & $\mathrm{MN}$ & Integumentary & 51 \\
\hline 1622 & Open wound of foot except toe(s) alon... & 8921 & 22485 & $\mathrm{MN}$ & Integumentary & 47 \\
\hline 1623 & Onychia and paronychia of finger & 68102 & 58220 & $\mathrm{MN}$ & Integumentary & 47 \\
\hline 1624 & Other specified disorders of penis & 60789 & 6503 & $\mathrm{MN}$ & Reproductive.Male & 33 \\
\hline 1626 & Pregnant state, incidental & V222 & 3498 & $\mathrm{MN}$ & Reproductive.Female & 45 \\
\hline 1627 & Impulse control disorder, unspecified & 31230 & 1981 & $\mathrm{MN}$ & Psychogenic & 42 \\
\hline 1628 & Unspecified adjustment reaction & 3099 & 34963 & $\mathrm{MN}$ & Psychogenic & 47 \\
\hline 1629 & Acute upper respiratory infections of. . . & 4659 & 5828386 & $\mathrm{MN}$ & Respiratory & 38 \\
\hline 1630 & Vaginitis and vulvovaginitis, unspeci... & 61610 & 713180 & $\mathrm{MN}$ & Reproductive.Female & 47 \\
\hline 1632 & Other specified gastritis without men... & 53540 & 6783 & $\mathrm{MN}$ & Gastrointestinal & 47 \\
\hline 1633 & Traumatic arthropathy, lower leg & 71616 & 27114 & $\mathrm{MN}$ & Musculoskeletal & 51 \\
\hline 1634 & Thoracic aneurysm without mention of ... & 4412 & 22404 & $\mathrm{MN}$ & Cardiovascular & 70 \\
\hline 1635 & Diaphragmatic hernia & 5533 & 70948 & $\mathrm{MN}$ & Gastrointestinal & 33 \\
\hline 1636 & Other specified disorders of joint, $1 . \ldots$ & 71986 & 37226 & $\mathrm{MN}$ & Musculoskeletal & 39 \\
\hline 1637 & Diverticulosis of colon (without ment. . & 56210 & 171796 & $\mathrm{MN}$ & Gastrointestinal & 33 \\
\hline 1638 & Closed fracture of unspecified part o... & 82380 & 58267 & $\mathrm{MN}$ & Musculoskeletal & 56 \\
\hline 1639 & Fitting and adjustment of orthopedic ... & V537 & 14090 & $\mathrm{MN}$ & Musculoskeletal & 28 \\
\hline 1640 & Ganglion of tendon sheath & 72742 & 17295 & $\mathrm{MN}$ & Musculoskeletal & 38 \\
\hline 1641 & Facial weakness & 78194 & 8137 & $\mathrm{MN}$ & Neurologic & 70 \\
\hline 1642 & Insomnia due to mental disorder & 32702 & 69849 & $\mathrm{MN}$ & Psychogenic & 51 \\
\hline 1643 & Blepharitis, unspecified & 37300 & 96642 & $\mathrm{EC}$ & Special.Sensory & 36 \\
\hline 1645 & Lipoma, unspecified site & 2149 & 144389 & $\mathrm{MN}$ & Integumentary & 22 \\
\hline 1646 & Enterobiasis & 1274 & 4625 & $\mathrm{MN}$ & Gastrointestinal & 42 \\
\hline 1648 & Diabetes with renal manifestations, t. . & 25042 & 104352 & $\mathrm{EC}$ & Endocrine & 60 \\
\hline 1649 & Other chronic disease of tonsils and ... & 4748 & 8459 & $\mathrm{EC}$ & Respiratory & 39 \\
\hline 1650 & Panic disorder & 30001 & 501214 & $\mathrm{MN}$ & Psychogenic & 60 \\
\hline 1651 & Dysfunction of Eustachian tube & 38181 & 476864 & $\mathrm{MN}$ & Special.Sensory & 28 \\
\hline 1652 & Viral hepatitis B w/o hepatc coma, ch. . & 7032 & 43115 & $\mathrm{MN}$ & Gastrointestinal & 65 \\
\hline 1653 & Scoliosis [and kyphoscoliosis], idiop... & 73730 & 185186 & $\mathrm{MN}$ & Musculoskeletal & 38 \\
\hline 1654 & Ingrowing nail & 7030 & 255436 & $\mathrm{MN}$ & Integumentary & 33 \\
\hline 1655 & Other specified hypertrophic and atro. . & 7018 & 17594 & $\mathrm{EC}$ & Integumentary & 31 \\
\hline 1656 & Bronchopneumonia, organism unspecified & 485 & 41274 & $\mathrm{MN}$ & Respiratory & 65 \\
\hline 1657 & Special screeng for oth disorders of ... & V788 & 28430 & $\mathrm{EC}$ & Hematologic.Immune & 31 \\
\hline 1658 & Chronic kidney disease, Stage III(modera & 5853 & 148351 & $\mathrm{MN}$ & Nephrologic & 51 \\
\hline 1659 & Other examination of ears and hearing & V7219 & 35801 & EC & Special.Sensory & 45 \\
\hline 1660 & Acute laryngopharyngitis & 4650 & 51342 & $\mathrm{EC}$ & Respiratory & 33 \\
\hline 1661 & Hypotension, unspecified & 4589 & 160413 & $\mathrm{MN}$ & Cardiovascular & 68 \\
\hline 1662 & Hereditary progressive muscular dystr. . & 3591 & 81718 & EC & Musculoskeletal & 60 \\
\hline 1663 & Special screening examination for pul. . & V741 & 210656 & $\mathrm{EC}$ & Respiratory & 39 \\
\hline 1664 & Unspecified transient cerebral ischemia & 4359 & 224878 & $\mathrm{MN}$ & Neurologic & 82 \\
\hline 1665 & Tinea blanca & 1112 & 46806 & $\mathrm{MN}$ & Integumentary & 15 \\
\hline 1666 & Abnormal underweight & 78322 & 18364 & $\mathrm{MN}$ & Nonspecific & 56 \\
\hline
\end{tabular}




\begin{tabular}{|c|c|c|c|c|c|c|}
\hline Item & ICD-9 Description & ICD-9 Code & $\begin{array}{l}\text { Weighted } \\
\text { Frequency }\end{array}$ & $\begin{array}{l}\text { Decision } \\
\text { Round }\end{array}$ & TPS Category & $\mathrm{IoH}$ \\
\hline 1667 & Unspecifiedisorder of prostate & 6029 & 54251 & $\mathrm{MN}$ & Reproductive.Male & 39 \\
\hline 1668 & Other specified disorders of circulat. & 45989 & 66331 & $\mathrm{MN}$ & Cardiovascular & 45 \\
\hline 1669 & Lumbar intervertebral disc without my. . . & 72210 & 286052 & $\mathrm{MN}$ & Musculoskeletal & 42 \\
\hline 1670 & Infective otitis externa, unspecified & 38010 & 486084 & $\mathrm{MN}$ & Special.Sensory & 42 \\
\hline 1671 & Open wound of face, unspecified site & 87340 & 36567 & $\mathrm{MN}$ & Integumentary & 56 \\
\hline 1672 & Acne varioliformis & 7060 & 5126 & $\mathrm{MN}$ & Integumentary & 28 \\
\hline 1673 & Essential and other specified forms o... & 3331 & 85528 & $\mathrm{EC}$ & Neurologic & 43 \\
\hline 1674 & Other emphysema & 4928 & 88212 & $\mathrm{MN}$ & Respiratory & 56 \\
\hline 1675 & Eating disorder, unspecified & 30750 & 89350 & $\mathrm{MN}$ & Psychogenic & 56 \\
\hline 1676 & Rheumatism, unspecified and fibrositis & 7290 & 27114 & $\mathrm{MN}$ & Musculoskeletal & 42 \\
\hline 1677 & Unspecified neurotic disorder & 3009 & 41179 & $\mathrm{MN}$ & Psychogenic & 38 \\
\hline 1678 & Other cellulitis and abscess of foot,... & 6827 & 93845 & $\mathrm{MN}$ & Integumentary & 60 \\
\hline 1679 & Pain in joint of pelvic region and thigh & 71945 & 1183355 & $\mathrm{MN}$ & Musculoskeletal & 42 \\
\hline 1681 & Burn [any deg] inv les thn $10 \%$ bdy su. & 94800 & 254382 & $\mathrm{MN}$ & Integumentary & 56 \\
\hline 1683 & Alopecia, unspecified & 70400 & 199709 & $\mathrm{MN}$ & Integumentary & 28 \\
\hline 1684 & Benign neoplasm of ovary & 220 & 15464 & $\mathrm{MN}$ & Reproductive.Female & 47 \\
\hline 1685 & Infection of kidney, unspecified & 5909 & 56743 & $\mathrm{MN}$ & Nephrologic & 65 \\
\hline 1686 & Abnormal glucose tolerance, antepartu. . & 64883 & 25622 & $\mathrm{MN}$ & Endocrine & 60 \\
\hline 1687 & Prophylactic vaccination/inoculation ... & V040 & 37630 & $\mathrm{MN}$ & Population.based.Care & 39 \\
\hline 1688 & Benign neoplasm of eye, part unspecified & 2249 & 6228 & $\mathrm{MN}$ & Special.Sensory & 51 \\
\hline 1689 & Unspecified acute reaction to stress & 3089 & 404573 & $\mathrm{MN}$ & Psychogenic & 51 \\
\hline 1690 & Cervical spondylosis with myelopathy & 7211 & 48270 & $\mathrm{EC}$ & Musculoskeletal & 60 \\
\hline 1691 & Teething syndrome & 5207 & 1807 & $\mathrm{EC}$ & Special.Sensory & 28 \\
\hline 1692 & $\begin{array}{l}\text { Drug induced headache, not elsewhere } \\
\text { classified }\end{array}$ & 3393 & 4574 & $\mathrm{MN}$ & Neurologic & 42 \\
\hline 1693 & Dementia in cond class elsewh w/o behavi & 29410 & 98583 & $\mathrm{MN}$ & Neurologic & 65 \\
\hline 1694 & Malignant neoplasm of larynx, unspeci. . . & 1619 & 21003 & $\mathrm{EC}$ & Respiratory & 92 \\
\hline 1695 & Attention or concentration deficit & 79951 & 8137 & $\mathrm{MN}$ & Psychogenic & 51 \\
\hline 1696 & Enteritis due to other organism, not... & 88 & 118357 & $\mathrm{MN}$ & Gastrointestinal & 42 \\
\hline 1697 & Blisters, epidermal loss [second degr... & 94522 & 28430 & $\mathrm{MN}$ & Integumentary & 56 \\
\hline 1698 & Unspecified mental retardation & 319 & 92593 & $\mathrm{EC}$ & Psychogenic & 51 \\
\hline 1700 & Other ventral hernia with obstruction & 55229 & 7437 & $\mathrm{MN}$ & Gastrointestinal & 75 \\
\hline 1702 & Chronic gouty arthropathy w/o tophus ... & 27402 & 44037 & $\mathrm{MN}$ & Musculoskeletal & 45 \\
\hline 1703 & Prophylac vacc/inocula agnst influenz. . & V0481 & 951972 & $\mathrm{MN}$ & Population.based.Care & 42 \\
\hline 1704 & Prophylac vacc/inocula agnst oth viral.. & V0489 & 132782 & $\mathrm{MN}$ & Population.based.Care & 42 \\
\hline 1705 & Other disorders of conjunctiva & 37289 & 28430 & $\mathrm{MN}$ & Special.Sensory & 30 \\
\hline 1707 & Unspecified disorder of male genital ... & 6089 & 124688 & $\mathrm{MN}$ & Reproductive.Male & 42 \\
\hline 1708 & Fitting and adjustment of vascular ca. . & V5881 & 5181 & $\mathrm{MN}$ & Cardiovascular & 48 \\
\hline 1709 & Other and unspecified angina pectoris & 4139 & 3424 & $\mathrm{MN}$ & Cardiovascular & 68 \\
\hline 1710 & Cervicobrachial syndrome (diffuse) & 7233 & 46806 & $\mathrm{MN}$ & Neurologic & 54 \\
\hline 1711 & Heartburn & 7871 & 84708 & $\mathrm{MN}$ & Gastrointestinal & 49 \\
\hline 1712 & Leiomyoma of uterus, unspecified & 2189 & 214103 & $\mathrm{MN}$ & Reproductive.Female & 49 \\
\hline 1713 & Viral hepatitis B w/o hep coma, acute. . & 7030 & 4159 & $\mathrm{MN}$ & Gastrointestinal & 68 \\
\hline 1714 & Unspecified suppurative otitis media & 3824 & 6911 & $\mathrm{MN}$ & Special.Sensory & 45 \\
\hline 1715 & Lumbago & 7242 & 4527666 & $\mathrm{MN}$ & Musculoskeletal & 36 \\
\hline 1716 & Squamous cell carcinoma of skin, site unspec & 17392 & 15228 & $\mathrm{MN}$ & Integumentary & 68 \\
\hline 1717 & Varices of other sites & 4568 & 9606 & $\mathrm{MN}$ & Cardiovascular & 40 \\
\hline 1718 & Malignant neoplasm of breast (female). . . & 1749 & 369607 & $\mathrm{EC}$ & Reproductive.Female & 91 \\
\hline
\end{tabular}




\begin{tabular}{|c|c|c|c|c|c|c|}
\hline Item & ICD-9 Description & ICD-9 Code & $\begin{array}{l}\text { Weighted } \\
\text { Frequency }\end{array}$ & $\begin{array}{l}\text { Decision } \\
\text { Round }\end{array}$ & TPS Category & $\mathrm{IoH}$ \\
\hline 1719 & Acute renal failure, unspecified & 5849 & 71964 & $\mathrm{MN}$ & Nephrologic & 81 \\
\hline 1720 & Moderate mental retardation & 3180 & 3185 & $\mathrm{EC}$ & Psychogenic & 54 \\
\hline 1721 & Olecranon bursitis & 72633 & 50698 & $\mathrm{MN}$ & Musculoskeletal & 45 \\
\hline 1722 & Other premature beats & 42769 & 37930 & $\mathrm{MN}$ & Cardiovascular & 40 \\
\hline 1723 & Arthrodesis status & V454 & 62329 & $\mathrm{MN}$ & Musculoskeletal & 13 \\
\hline 1724 & Routine gynecological examination & V7231 & 2219176 & $\mathrm{MN}$ & Reproductive.Female & 42 \\
\hline 1725 & Other screening breast examination fo... & V7619 & 29882 & $\mathrm{EC}$ & Reproductive.Female & 30 \\
\hline 1726 & Paroxysmal supraventricular tachycardia & 4270 & 37758 & $\mathrm{MN}$ & Cardiovascular & 72 \\
\hline 1727 & Acute cystitis & 5950 & 143753 & $\mathrm{EC}$ & Nephrologic & 45 \\
\hline 1728 & Generalized pain & 78096 & 409905 & $\mathrm{MN}$ & Nonspecific & 49 \\
\hline 1730 & Atherosclerosis of the extremities w/... & 44021 & 48429 & $\mathrm{MN}$ & Cardiovascular & 40 \\
\hline 1732 & $\begin{array}{l}\text { Removal and reinsertion of intrauterine } \\
\text { cont... }\end{array}$ & V2513 & 16002 & $\mathrm{MN}$ & Reproductive.Female & 42 \\
\hline 1733 & Helicobacter pylori (H. pylori) infec. . . & 4186 & 75694 & $\mathrm{MN}$ & Gastrointestinal & 45 \\
\hline 1734 & Psychosexual dysfunction with prematu... & 30275 & 33588 & $\mathrm{MN}$ & Psychogenic & 45 \\
\hline 1735 & Other symptoms involving digestive sy... & 78799 & 36203 & $\mathrm{MN}$ & Gastrointestinal & 36 \\
\hline 1738 & Other and unspecified disc disorder, ... & 72290 & 107460 & $\mathrm{MN}$ & Musculoskeletal & 40 \\
\hline 1739 & Other convulsions & 78039 & 299465 & $\mathrm{MN}$ & Neurologic & 68 \\
\hline 1740 & Sprains and strains of other specifie... & 8448 & 37630 & $\mathrm{MN}$ & Musculoskeletal & 40 \\
\hline 1741 & Disorders of bursae and tendons in sh... & 72610 & 462697 & $\mathrm{MN}$ & Musculoskeletal & 40 \\
\hline 1742 & Other, mixed, or unspecified drug abu. .. & 30593 & 12393 & $\mathrm{MN}$ & Psychogenic & 58 \\
\hline 1743 & Myopathy, unspecified & 3599 & 27844 & $\mathrm{MN}$ & Musculoskeletal & 54 \\
\hline 1745 & Other tenosynovitis or hand and wrist & 72705 & 56443 & $\mathrm{MN}$ & Musculoskeletal & 45 \\
\hline 1747 & Enteritis of unspecified site & 5559 & 213382 & $\mathrm{MN}$ & Gastrointestinal & 40 \\
\hline 1748 & Other orthopedic aftercare & V5489 & 42133 & $\mathrm{MN}$ & Musculoskeletal & 36 \\
\hline 1749 & Corneal foreign body & 9300 & 39483 & $\mathrm{MN}$ & Special.Sensory & 49 \\
\hline 1750 & Metrorrhagia & 6266 & 19601 & $\mathrm{MN}$ & Reproductive.Female & 49 \\
\hline 1751 & Dysmenorrhea & 6253 & 183471 & $\mathrm{MN}$ & Reproductive.Female & 36 \\
\hline 1752 & Sprains and strains of thoracic & 8471 & 221472 & $\mathrm{MN}$ & Musculoskeletal & 36 \\
\hline 1753 & Other seborrheic dermatitis & 69018 & 22518 & $\mathrm{MN}$ & Integumentary & 26 \\
\hline 1754 & Open fracture of metacarpal bone(s), ... & 81510 & 12081 & $\mathrm{MN}$ & Musculoskeletal & 63 \\
\hline 1755 & Preoperative cardiovascular examination & V7281 & 38593 & $\mathrm{MN}$ & Cardiovascular & 59 \\
\hline 1756 & Contusion of knee & 92411 & 80198 & $\mathrm{MN}$ & Musculoskeletal & 26 \\
\hline 1757 & Secondary hyperparathyroidism(of renal.. & 58881 & 15474 & $\mathrm{MN}$ & Endocrine & 49 \\
\hline 1758 & Malignant neoplasm of renal pelvis & 1891 & 17594 & $\mathrm{MN}$ & Nephrologic & 81 \\
\hline 1760 & Foreign body in digestive system, uns. . & 938 & 6648 & $\mathrm{MN}$ & Gastrointestinal & 49 \\
\hline 1761 & $\begin{array}{l}\text { Other screening mammogram for } \\
\text { maligna... }\end{array}$ & V7612 & 158536 & $\mathrm{EC}$ & Reproductive.Female & 48 \\
\hline 1763 & Laboratory examination ordered as part. . & V7262 & 76283 & $\mathrm{MN}$ & Nonspecific & 36 \\
\hline 1764 & Ulcer of heel and midfoot, exc decubitus & 70714 & 5181 & $\mathrm{MN}$ & Integumentary & 49 \\
\hline 1765 & Unspecified intestinal obstruction & 5609 & 65725 & $\mathrm{MN}$ & Gastrointestinal & 73 \\
\hline 1766 & Atherosclerosis of other specified ar... & 4408 & 7828 & $\mathrm{MN}$ & Cardiovascular & 45 \\
\hline 1767 & Closed fracture of middle or proximal. . . & 81601 & 28430 & $\mathrm{MN}$ & Musculoskeletal & 54 \\
\hline 1768 & $\begin{array}{l}\text { Personal history of other musculoskeletal } \\
\text { disorders }\end{array}$ & V1359 & 1985 & $\mathrm{MN}$ & Musculoskeletal & 24 \\
\hline 1769 & Other anomalies lower limb, including. . . & 75569 & 3298 & $\mathrm{MN}$ & Musculoskeletal & 30 \\
\hline 1770 & Bacterial enteritis, unspecified & 85 & 28430 & $\mathrm{MN}$ & Gastrointestinal & 49 \\
\hline 1771 & Elbow, forearm, and wrist injury, oth. . & 9593 & 90423 & $\mathrm{MN}$ & Musculoskeletal & 38 \\
\hline 1772 & Anemia, unspecified & 2859 & 1496928 & $\mathrm{MN}$ & Hematologic.Immune & 49 \\
\hline 1775 & Other closed fractures of distal end ... & 81342 & 12464 & $\mathrm{MN}$ & Musculoskeletal & 54 \\
\hline
\end{tabular}




\begin{tabular}{|c|c|c|c|c|c|c|}
\hline Item & ICD-9 Description & ICD-9 Code & $\begin{array}{l}\text { Weighted } \\
\text { Frequency }\end{array}$ & $\begin{array}{l}\text { Decision } \\
\text { Round }\end{array}$ & TPS Category & $\mathrm{IoH}$ \\
\hline 1778 & Pityriasis versicolor & 1110 & 39643 & $\mathrm{MN}$ & Integumentary & 26 \\
\hline 1779 & $\begin{array}{l}\text { Family history of Oth musculoskeletal } \\
\text { diseases }\end{array}$ & V1789 & 1985 & $\mathrm{MN}$ & Musculoskeletal & 13 \\
\hline 1780 & Screening mammogram for high-risk pat. . & V7611 & 34771 & EC & Reproductive.Female & 48 \\
\hline 1781 & Hip replacement & V4364 & 10449 & $\mathrm{MN}$ & Musculoskeletal & 36 \\
\hline 1783 & Headache & 7840 & 2290819 & $\mathrm{MN}$ & Neurologic & 49 \\
\hline 1784 & Oppositional disorder & 31381 & 23075 & $\mathrm{MN}$ & Psychogenic & 40 \\
\hline 1785 & Dysmetabolic syndrome X & 2777 & 569545 & $\mathrm{MN}$ & Endocrine & 40 \\
\hline 1786 & Carbuncle and furuncle of unspecified. . & 6809 & 186372 & $\mathrm{MN}$ & Integumentary & 31 \\
\hline 1787 & Other voice disturbance & 78449 & 25279 & $\mathrm{EC}$ & Respiratory & 49 \\
\hline 1788 & Osteoarthrosis, unspec generalized or... & 71590 & 2630345 & $\mathrm{MN}$ & Musculoskeletal & 26 \\
\hline 1789 & Paranoid personality disorder & 3010 & 6466 & $\mathrm{MN}$ & Psychogenic & 49 \\
\hline 1793 & Unspecified local infection of skin a. & 6869 & 117843 & $\mathrm{MN}$ & Integumentary & 36 \\
\hline 1794 & Nephritis/nephropathy w/unspec pathol. . . & 5839 & 42249 & $\mathrm{MN}$ & Nephrologic & 54 \\
\hline 1795 & Unspecified contusion of eye & 9219 & 1985 & $\mathrm{MN}$ & Special.Sensory & 58 \\
\hline 1796 & Lumbar region spondylosis with myelop. . . & 72142 & 4201 & $\mathrm{EC}$ & Musculoskeletal & 54 \\
\hline 1798 & Mechanical complication of tracheostomy & 51902 & 12176 & $\mathrm{EC}$ & Respiratory & 42 \\
\hline 1799 & Sprains and strains of medial collate... & 8441 & 49991 & $\mathrm{MN}$ & Musculoskeletal & 45 \\
\hline 1800 & Chronic prostatitis & 6011 & 18303 & $\mathrm{EC}$ & Reproductive.Male & 45 \\
\hline 1801 & Unspecified disorder of eye movements & 3789 & 3415 & $\mathrm{MN}$ & Special.Sensory & 43 \\
\hline 1802 & Insertion of intrauterine contraceptive device & V2511 & 58105 & $\mathrm{MN}$ & Reproductive.Female & 39 \\
\hline 1803 & $\begin{array}{l}\text { Asymptomatic human } \\
\text { immunodeficiency v. . . }\end{array}$ & V08 & 19549 & $\mathrm{MN}$ & Hematologic.Immune & 70 \\
\hline 1804 & Cerebral thrombosis with cerebral inf. . & 43401 & 11398 & $\mathrm{MN}$ & Neurologic & 82 \\
\hline 1805 & Lesion of radial nerve & 3543 & 11829 & $\mathrm{MN}$ & Neurologic & 58 \\
\hline 1807 & Seroma complicating a procedure & 99813 & 11942 & $\mathrm{MN}$ & Integumentary & 31 \\
\hline 1808 & Unspecified anomaly of heart & 7469 & 8187 & $\mathrm{MN}$ & Cardiovascular & 54 \\
\hline 1809 & Solitary cyst of breast & 6100 & 7687 & $\mathrm{EC}$ & Reproductive.Female & 43 \\
\hline 1810 & Chronic lymphocytic thyroiditis & 2452 & 83762 & $\mathrm{MN}$ & Endocrine & 47 \\
\hline 1811 & Injury to other specified sites, incl. . & 9598 & 20384 & $\mathrm{MN}$ & Nonspecific & 40 \\
\hline 1812 & Precordial pain & 78651 & 17420 & $\mathrm{MN}$ & Cardiovascular & 62 \\
\hline 1814 & Sprains and strains of carpal (joint) & 84201 & 7437 & $\mathrm{MN}$ & Musculoskeletal & 35 \\
\hline 1815 & Synovial cyst, unspecified & 72740 & 7437 & $\mathrm{MN}$ & Musculoskeletal & 39 \\
\hline 1816 & Intrauterine death, delivered with or. . & 65641 & 29644 & $\mathrm{MN}$ & Reproductive.Female & 70 \\
\hline 1817 & Plantar wart & 7812 & 146421 & $\mathrm{MN}$ & Integumentary & 18 \\
\hline 1818 & Unspecified monoarthritis, lower leg & 71666 & 18233 & $\mathrm{MN}$ & Musculoskeletal & 43 \\
\hline 1819 & Open wound of vagina, without mention. . . & 8786 & 15464 & $\mathrm{MN}$ & Reproductive.Female & 50 \\
\hline 1820 & $\begin{array}{l}\text { Squamous cell carcinoma skin upper limb, } \\
\text { inc shoulder }\end{array}$ & 17362 & 28430 & $\mathrm{MN}$ & Integumentary & 62 \\
\hline 1821 & Open wound of auditory canal & 87202 & 16002 & $\mathrm{EC}$ & Special.Sensory & 47 \\
\hline 1822 & Mononeuritis of lower limb, unspecified & 3558 & 28938 & $\mathrm{MN}$ & Neurologic & 50 \\
\hline 1823 & Effusion of joint of forearm & 71903 & 9344 & $\mathrm{MN}$ & Musculoskeletal & 43 \\
\hline 1824 & Ulcer of lower limbs, exc decubitus, uns & 70710 & 69026 & $\mathrm{MN}$ & Integumentary & 43 \\
\hline 1826 & Unspecified pruritic disorder & 6989 & 220050 & $\mathrm{MN}$ & Integumentary & 47 \\
\hline 1827 & Nontoxic uninodular goiter & 2410 & 142001 & $\mathrm{MN}$ & Endocrine & 43 \\
\hline 1828 & Unspecified disorder of kidney and ur. . . & 5939 & 277851 & $\mathrm{MN}$ & Nephrologic & 49 \\
\hline 1829 & Major depressive disorder, single epi... & 29622 & 11398 & $\mathrm{MN}$ & Psychogenic & 50 \\
\hline 1830 & Other forms of retinal detachment & 36189 & 27114 & $\mathrm{MN}$ & Special.Sensory & 87 \\
\hline 1831 & Pleurisy unspecified pleural effusion & 5119 & 74075 & $\mathrm{MN}$ & Respiratory & 60 \\
\hline
\end{tabular}




\begin{tabular}{|c|c|c|c|c|c|c|}
\hline Item & ICD-9 Description & ICD-9 Code & $\begin{array}{l}\text { Weighted } \\
\text { Frequency }\end{array}$ & $\begin{array}{l}\text { Decision } \\
\text { Round }\end{array}$ & TPS Category & $\mathrm{IoH}$ \\
\hline 1832 & Congenital dislocation of hip, bilateral & 75431 & 3789 & $\mathrm{MN}$ & Musculoskeletal & 58 \\
\hline 1835 & Other synovitis and tenosynovitis & 72709 & 111610 & $\mathrm{MN}$ & Musculoskeletal & 47 \\
\hline 1836 & Other enthesopathy of knee & 72669 & 11398 & $\mathrm{MN}$ & Musculoskeletal & 43 \\
\hline 1837 & Osteoarthrosis, unspec generalized or. . & 71591 & 73920 & $\mathrm{MN}$ & Musculoskeletal & 39 \\
\hline 1838 & Hypoxemia & 79902 & 143650 & $\mathrm{MN}$ & Respiratory & 70 \\
\hline 1839 & Special screening for malignant neopl. . & V7643 & 7437 & EC & Integumentary & 54 \\
\hline 1840 & Other abnormal blood chemistry & 7906 & 192663 & $\mathrm{MN}$ & Nonspecific & 36 \\
\hline 1842 & Abscess of Bartholin`s gland & 6163 & 28430 & $\mathrm{MN}$ & Reproductive.Female & 58 \\
\hline 1843 & Lump or mass in breast & 61172 & 275096 & $\mathrm{MN}$ & Reproductive.Female & 62 \\
\hline 1844 & Opioid type dependence, continuous & 30401 & 91370 & $\mathrm{MN}$ & Psychogenic & 62 \\
\hline 1845 & Whooping cough, unspecified organism & 339 & 29838 & $\mathrm{MN}$ & Respiratory & 58 \\
\hline 1846 & Other specified iron deficiency anemias & 2808 & 3185 & $\mathrm{MN}$ & Hematologic.Immune & 54 \\
\hline 1847 & Constipation, unspecified & 56400 & 1148884 & $\mathrm{MN}$ & Gastrointestinal & 39 \\
\hline 1848 & Prtial epilepsy, w/impair of consciou. . . & 34540 & 6350 & $\mathrm{MN}$ & Neurologic & 70 \\
\hline 1849 & Acute gastritis without mention of ob... & 53500 & 48972 & $\mathrm{MN}$ & Gastrointestinal & 50 \\
\hline 1850 & Observation for abuse and neglect & V7181 & 27114 & EC & Patient.based.Systems & 58 \\
\hline 1851 & Tachycardia, unspecified & 7850 & 213802 & $\mathrm{MN}$ & Cardiovascular & 62 \\
\hline 1852 & Chronic airway obstruction, not elsew... & 496 & 2389035 & $\mathrm{MN}$ & Respiratory & 58 \\
\hline 1854 & Other specified disorders of joint, u. . . & 71982 & 7582 & $\mathrm{MN}$ & Musculoskeletal & 36 \\
\hline 1855 & Prolonged posttraumatic stress disorder & 30981 & 140561 & $\mathrm{MN}$ & Psychogenic & 58 \\
\hline 1856 & Other acquired deformities of ankle a... & 73679 & 12247 & $\mathrm{MN}$ & Musculoskeletal & 39 \\
\hline 1857 & $\begin{array}{l}\text { Unspec malignant neoplasm of skin, site } \\
\text { unspec }\end{array}$ & 17390 & 16002 & $\mathrm{MN}$ & Integumentary & 66 \\
\hline 1858 & Pain in joint of hand & 71944 & 117256 & $\mathrm{MN}$ & Musculoskeletal & 43 \\
\hline 1859 & Disorders of sacrum & 7246 & 62808 & $\mathrm{MN}$ & Musculoskeletal & 36 \\
\hline 1860 & Explosive personality disorder & 3013 & 4382 & $\mathrm{MN}$ & Psychogenic & 43 \\
\hline 1861 & Acute bronchiolitis due to other infe... & 46619 & 108193 & $\mathrm{MN}$ & Respiratory & 62 \\
\hline 1862 & Benign neoplasm of skin of lower limb... & 2167 & 29644 & $\mathrm{MN}$ & Integumentary & 27 \\
\hline 1864 & Other hemochromatosis & 27503 & 35825 & EC & Hematologic.Immune & 58 \\
\hline 1865 & Anal or rectal pain & 56942 & 14203 & $\mathrm{MN}$ & Gastrointestinal & 47 \\
\hline 1866 & Impacted cerumen & 3804 & 721552 & $\mathrm{MN}$ & Special.Sensory & 31 \\
\hline 1867 & Unspecified testicular dysfunction & 2579 & 14342 & $\mathrm{MN}$ & Reproductive.Male & 59 \\
\hline 1868 & Secondary malignant neoplasm of bone ... & 1985 & 3798 & $\mathrm{MN}$ & Musculoskeletal & 75 \\
\hline 1869 & Other disorders of synovium, tendon, ... & 72789 & 42802 & $\mathrm{MN}$ & Musculoskeletal & 40 \\
\hline 1871 & Abdominal tenderness, right upper qua. . & 78961 & 2491 & $\mathrm{MN}$ & Gastrointestinal & 58 \\
\hline 1872 & Other and unspecified anomalies of mu... & 7569 & 37630 & $\mathrm{EC}$ & Musculoskeletal & 59 \\
\hline 1873 & Old myocardial infarction & 412 & 37630 & $\mathrm{MN}$ & Cardiovascular & 62 \\
\hline 1874 & Preoperative examination, unspecified & V7284 & 520750 & $\mathrm{MN}$ & Nonspecific & 49 \\
\hline 1875 & Special screening malig neopl of colon & V7651 & 219463 & $\mathrm{EC}$ & Gastrointestinal & 47 \\
\hline 1876 & Mastodynia & 61171 & 165805 & $\mathrm{MN}$ & Reproductive.Female & 39 \\
\hline 1877 & Other anaphylactic shock & 9950 & 55543 & EC & Nonspecific & 92 \\
\hline 1878 & Gastrojejunal ulcer unspec acut/chron. & 53490 & 11463 & $\mathrm{MN}$ & Gastrointestinal & 62 \\
\hline 1879 & Seborrheic dermatitis, unspecified & 69010 & 45840 & $\mathrm{MN}$ & Integumentary & 22 \\
\hline 1880 & Other acute reactions to stress & 3083 & 22913 & $\mathrm{MN}$ & Psychogenic & 47 \\
\hline 1881 & Intestinal parasitism, unspecified & 129 & 37630 & $\mathrm{MN}$ & Gastrointestinal & 50 \\
\hline 1882 & Unspecified degree burn of ankle & 94503 & 2967 & $\mathrm{MN}$ & Integumentary & 49 \\
\hline 1883 & Other specified sites of sprains and ... & 8488 & 54365 & $\mathrm{MN}$ & Musculoskeletal & 36 \\
\hline
\end{tabular}


Appendix A Continued

\begin{tabular}{|c|c|c|c|c|c|c|}
\hline Item & ICD-9 Description & ICD-9 Code & $\begin{array}{l}\text { Weighted } \\
\text { Frequency }\end{array}$ & $\begin{array}{c}\text { Decision } \\
\text { Round }\end{array}$ & TPS Category & $\mathrm{IoH}$ \\
\hline 1884 & Acquired absence of both cervix and uterus & V8801 & 45825 & $\mathrm{MN}$ & Reproductive.Female & 27 \\
\hline 1885 & Sunburn & 69271 & 7691 & $\mathrm{MN}$ & Integumentary & 26 \\
\hline 1886 & Other contraceptive method & V2549 & 110904 & $\mathrm{MN}$ & Reproductive.Female & 39 \\
\hline 1887 & Other color vision deficiencies & 36859 & 6196 & $\mathrm{MN}$ & Special.Sensory & 27 \\
\hline 1888 & Other bursitis & 7273 & 146064 & $\mathrm{MN}$ & Musculoskeletal & 39 \\
\hline 1890 & Contact dermatitis/eczema of due to p... & 6926 & 389724 & $\mathrm{MN}$ & Integumentary & 33 \\
\hline 1892 & Other nerve root and plexus disorders & 3538 & 56859 & $\mathrm{MN}$ & Neurologic & 49 \\
\hline 1893 & Esophageal varices without mention of. . & 4561 & 27499 & $\mathrm{MN}$ & Gastrointestinal & 54 \\
\hline
\end{tabular}

ICD-9, International Classification of Diseases, Ninth Revision; IoH, Index of Harm; TPS, test plan specifications. 
Appendix B: ICD-9 Codes Removed from the Final Dataset $(\mathbf{n}=198)$

\begin{tabular}{|c|c|c|c|c|c|c|}
\hline Item & ICD-9 Description & $\begin{array}{l}\text { ICD-9 } \\
\text { Code }\end{array}$ & $\begin{array}{l}\text { Weighted } \\
\text { Frequency }\end{array}$ & $\begin{array}{l}\text { Decision } \\
\text { Round }\end{array}$ & Reason Excluded & $\mathrm{IoH}$ \\
\hline 9 & Other postsurgical status & V4589 & 278112 & EC & Too.Vague & 54 \\
\hline 11 & Other specified diseases due to Chlam... & 7888 & 12888 & EC & Too.Vague & 48 \\
\hline 16 & Unspecified nutritional deficiency & 2699 & 2557 & $\mathrm{EC}$ & Too.Vague & 42 \\
\hline 29 & Malignant neoplasm of other and unspe... & 1759 & 7519 & $\mathrm{EC}$ & Too.Vague & 75 \\
\hline 30 & Special screening for unspecified mal. & V769 & 54227 & $\mathrm{EC}$ & Too.Vague & 46 \\
\hline 35 & Other postoperative infection & 99859 & 45457 & $\mathrm{EC}$ & Too.Vague & 65 \\
\hline 41 & Family history of other specified mal. . . & V168 & 37630 & EC & Too.Vague & 25 \\
\hline 49 & Special screening for other and unspe... & V781 & 27114 & $\mathrm{EC}$ & Too.Vague & 30 \\
\hline 56 & Fertility testing & V2621 & 4372 & $\mathrm{EC}$ & Too.Vague & 37 \\
\hline 63 & Candidiasis of other urogenital sites & 1122 & 18558 & $\mathrm{EC}$ & Too.Vague & 36 \\
\hline 69 & Family hx of malignant neoplasm ... & V160 & 1522 & $\mathrm{EC}$ & Too.Vague & 36 \\
\hline 87 & Other specified noninflammatory disor. . & 6238 & 84448 & $\mathrm{EC}$ & Too.Vague & 26 \\
\hline 96 & Anaphylactic shock due to tree nuts a... & 99564 & 12776 & $\mathrm{EC}$ & No.Consensus & 89 \\
\hline 99 & Body Mass Index 26.0 to 26.9 , adult & V8522 & 35144 & $\mathrm{EC}$ & No.Consensus & 27 \\
\hline 102 & Other specified disorders involving t. . . & 2798 & 4593 & $\mathrm{EC}$ & No.Consensus & 34 \\
\hline 110 & Nocturia & 78843 & 129444 & $\mathrm{EC}$ & No.Consensus & 33 \\
\hline 114 & Malignant neoplasm other w/o specific. . . & 1991 & 115965 & $\mathrm{EC}$ & Too.Vague & 82 \\
\hline 119 & Acute gonococcal infection of lower g. . . & 980 & 80421 & $\mathrm{EC}$ & Too.Vague & 53 \\
\hline 121 & Family hx of malignant neoplasm ... & V163 & 9508 & EC & Too.Vague & 38 \\
\hline 149 & $\begin{array}{l}\text { Herpes simplex without mention of } \\
\text { com. . }\end{array}$ & 549 & 266826 & $\mathrm{EC}$ & Too.Vague & 42 \\
\hline 152 & Inj to mult and unsp intrathor orgn w. . & 8628 & 16224 & $\mathrm{EC}$ & Too.Vague & 68 \\
\hline 154 & Noncodable diagnosis/insuff info for ... & V990 & 1461460 & $\mathrm{EC}$ & Too.Vague & 5 \\
\hline 156 & Abdominal or pelvic swelling, mass, o... & 78932 & 7643 & $\mathrm{EC}$ & Too.Vague & 53 \\
\hline 177 & Malig neoplasm of connectiv/oth soft ... & 1719 & 9122 & $\mathrm{EC}$ & Too.Vague & 78 \\
\hline 182 & Other stomatitis and mucositis (ulcerative) & 52809 & 6228 & $\mathrm{EC}$ & No.Consensus & 42 \\
\hline 191 & Swelling, mass, or lump in head and neck & 7842 & 120116 & $\mathrm{EC}$ & Too.Vague & 44 \\
\hline 196 & Sterilization & V252 & 24057 & $\mathrm{EC}$ & Too.Vague & 43 \\
\hline 199 & Gout, unspecified & 2749 & 907988 & $\mathrm{EC}$ & No.Consensus & 49 \\
\hline 203 & Hemophilus influenza, type B [Hib] & V0381 & 5656 & $\mathrm{EC}$ & No.Consensus & 59 \\
\hline 219 & Other specified counseling & V6549 & 473364 & $\mathrm{EC}$ & Too.Vague & 34 \\
\hline 222 & Stomatitis and mucositis, unspecified & 52800 & 5306 & $\mathrm{EC}$ & No.Consensus & 32 \\
\hline 227 & Personal history of malignant neoplas. . . & V1046 & 97016 & $\mathrm{EC}$ & Too.Vague & 51 \\
\hline 236 & Open wound of tooth (broken) & 87363 & 55288 & $\mathrm{EC}$ & No.Consensus & 41 \\
\hline 253 & Other adverse food reactions, NEC & 9957 & 24095 & $\mathrm{EC}$ & Too.Vague & 34 \\
\hline 269 & Chronic pain syndrome & 3384 & 1494376 & $\mathrm{EC}$ & No.Consensus & 62 \\
\hline 277 & Other specified chlamydial infection & 7988 & 15464 & $\mathrm{EC}$ & Too.Vague & 54 \\
\hline 279 & Deficiency of other vitamins & 2691 & 13439 & $\mathrm{EC}$ & Too.Vague & 42 \\
\hline 319 & Other B-complex deficiencies & 2662 & 193679 & $\mathrm{EC}$ & Too.Vague & 41 \\
\hline 326 & Hemorrhage, unspecified & 4590 & 52398 & $\mathrm{EC}$ & Too.Vague & 65 \\
\hline 327 & Giant cell arteritis & 4465 & 6737 & $\mathrm{EC}$ & No.Consensus & 70 \\
\hline 331 & Chronic pain due to trauma & 33821 & 17311 & $\mathrm{EC}$ & Too.Vague & 53 \\
\hline 336 & Diagnostic skin and sensitization tests & V727 & 17594 & $\mathrm{EC}$ & Too.Vague & 30 \\
\hline 346 & Contusion of unspecified part of uppe... & 9239 & 45638 & $\mathrm{EC}$ & No.Consensus & 20 \\
\hline 366 & Other injury of other sites of trunk & 95919 & 118538 & $\mathrm{EC}$ & Too.Vague & 38 \\
\hline 369 & Swelling, mass, or lump in chest & 7866 & 85370 & $\mathrm{EC}$ & Too.Vague & 45 \\
\hline 381 & Malignant neoplasm of lower limb, inc. . & 1713 & 28430 & $\mathrm{EC}$ & Too.Vague & 72 \\
\hline 383 & Unspecified vitamin deficiency & 2692 & 16399 & $\mathrm{EC}$ & Too.Vague & 41 \\
\hline
\end{tabular}




\begin{tabular}{|c|c|c|c|c|c|c|}
\hline Item & ICD-9 Description & $\begin{array}{l}\text { ICD-9 } \\
\text { Code }\end{array}$ & $\begin{array}{l}\text { Weighted } \\
\text { Frequency }\end{array}$ & $\begin{array}{l}\text { Decision } \\
\text { Round }\end{array}$ & Reason Excluded & $\mathrm{IoH}$ \\
\hline 387 & Special screening examination for oth. & V7389 & 58267 & $\mathrm{EC}$ & Too.Vague & 36 \\
\hline 396 & Open wound of knee, leg [except thigh. . & 8911 & 27114 & $\mathrm{EC}$ & Too.Vague & 43 \\
\hline 399 & Benign neoplasm of site unspecified & 2299 & 10426 & $\mathrm{EC}$ & Too.Vague & 12 \\
\hline 401 & Examination for medicolegal reasons & V704 & 19826 & $\mathrm{EC}$ & No.Consensus & 29 \\
\hline 409 & Inflammatory conditions & 5264 & 14370 & $\mathrm{EC}$ & Too.Vague & 40 \\
\hline 413 & Contact with or exposure to venereal & V016 & 47074 & $\mathrm{EC}$ & Too.Vague & 52 \\
\hline 417 & Counseling NOS & V6540 & 75836 & $\mathrm{EC}$ & Too.Vague & 40 \\
\hline 426 & Hypercalcemia & 27542 & 49616 & $\mathrm{EC}$ & No.Consensus & 60 \\
\hline 427 & Adult sexual abuse & 99583 & 16822 & $\mathrm{EC}$ & No.Consensus & 65 \\
\hline 430 & Morbid obesity & 27801 & 770970 & $\mathrm{EC}$ & No.Consensus & 63 \\
\hline 454 & $\begin{array}{l}\text { Personal history of oth allergy, oth than } \\
\text { med agents }\end{array}$ & V1509 & 28430 & $\mathrm{EC}$ & Too.Vague & 24 \\
\hline 459 & Dietary surveillance and counseling & V653 & 337026 & $\mathrm{EC}$ & No.Consensus & 44 \\
\hline 470 & Acquired absence of genital organs & V4577 & 32927 & $\mathrm{EC}$ & Too.Vague & 41 \\
\hline 475 & Other venereal dis due to Chlamydia t. . & 9950 & 6503 & $\mathrm{EC}$ & Too.Vague & 59 \\
\hline 476 & $\begin{array}{l}\text { Other specified conditions originating } \\
\text { perinatal period }\end{array}$ & 77989 & 33179 & $\mathrm{EC}$ & Too.Vague & 44 \\
\hline 479 & Organic insomnia, unspecified & 32700 & 18850 & $\mathrm{EC}$ & No.Consensus & 54 \\
\hline 487 & Chlamydia trachomatis & 9941 & 12081 & $\mathrm{EC}$ & No.Consensus & 54 \\
\hline 490 & Personal history of malignant neoplas. . . & V1009 & 46806 & $\mathrm{EC}$ & Too.Vague & 56 \\
\hline 491 & Contusion of back & 92231 & 83373 & $\mathrm{EC}$ & No.Consensus & 27 \\
\hline 495 & High-risk sexual behavior & V692 & 95710 & $\mathrm{EC}$ & No.Consensus & 54 \\
\hline 497 & Chest pain, unspecified & 78650 & 1394712 & $\mathrm{EC}$ & Too.Vague & 67 \\
\hline 498 & History of fall & V1588 & 13681 & $\mathrm{EC}$ & No.Consensus & 46 \\
\hline 502 & $\begin{array}{l}\text { Other infections specific to the perinatal } \\
\text { period }\end{array}$ & 77189 & 5008 & $\mathrm{EC}$ & Too.Vague & 54 \\
\hline 510 & Hypertrophy of tonsils alone & 47411 & 86503 & $\mathrm{EC}$ & No.Consensus & 33 \\
\hline 527 & Ear piercing & V503 & 23291 & $\mathrm{EC}$ & No.Consensus & 5 \\
\hline 528 & Body Mass Index between 19 to 24 , adult & V851 & 17572 & $\mathrm{EC}$ & No.Consensus & 4 \\
\hline 542 & Hernia of unspecified site & 5539 & 159988 & $\mathrm{EC}$ & Too.Vague & 40 \\
\hline 547 & Streptococcus, unspecified bacterial ... & 4100 & 14615 & $\mathrm{EC}$ & Too.Vague & 47 \\
\hline 550 & Reaction to spinal or lumbar puncture & 3490 & 3460 & $\mathrm{EC}$ & Too.Vague & 45 \\
\hline 579 & Laboratory examination, unspecified & V7260 & 15980 & $\mathrm{EC}$ & Too.Vague & 23 \\
\hline 594 & Unspecified septicemia & 389 & 37630 & $\mathrm{EC}$ & No.Consensus & 93 \\
\hline 603 & Systemic lupus erythematosus & 7100 & 168594 & $\mathrm{EC}$ & No.Consensus & 70 \\
\hline 627 & Personal history of noncompliance wit. . . & V1581 & 52542 & $\mathrm{EC}$ & No.Consensus & 44 \\
\hline 634 & Exposure to pot hazardous bodily fluids & V1585 & 117969 & $\mathrm{EC}$ & Too.Vague & 65 \\
\hline 639 & Other/unspec complications of medical. . . & 9999 & 9554 & $\mathrm{EC}$ & Too.Vague & 35 \\
\hline 643 & Allergic rhinitis due to food & 4771 & 27114 & $\mathrm{EC}$ & No.Consensus & 29 \\
\hline 651 & Arthropod-borne disease, unspecified & 889 & 28430 & $\mathrm{EC}$ & Too.Vague & 54 \\
\hline 659 & Varicella without mention of complica. . & 529 & 111509 & $\mathrm{EC}$ & No.Consensus & 46 \\
\hline 661 & Other replacement & V4369 & 9344 & $\mathrm{EC}$ & Too.Vague & 27 \\
\hline 681 & Observation following alleged rape or... & V715 & 4809 & $\mathrm{EC}$ & No.Consensus & 65 \\
\hline 682 & Other specified general medical exami... & V708 & 51330 & $\mathrm{EC}$ & No.Consensus & 42 \\
\hline 686 & Unspecified follow-up examination & V679 & 1367568 & $\mathrm{EC}$ & Too.Vague & 19 \\
\hline 701 & Nonallopathic lesions, abdomen and other & 7399 & 122158 & $\mathrm{EC}$ & No.Consensus & 25 \\
\hline 741 & Contusion of multiple sites, not else... & 9248 & 53026 & $\mathrm{EC}$ & Too.Vague & 32 \\
\hline 753 & Fracture of nasal bones, closed & 8020 & 46806 & $\mathrm{EC}$ & No.Consensus & 40 \\
\hline 764 & $\begin{array}{l}\text { Personal history Other disorders of } \\
\text { nervous system and. . }\end{array}$ & V1249 & 116088 & $\mathrm{EC}$ & Too.Vague & 44 \\
\hline
\end{tabular}




\begin{tabular}{|c|c|c|c|c|c|c|}
\hline Item & ICD-9 Description & $\begin{array}{l}\text { ICD-9 } \\
\text { Code }\end{array}$ & $\begin{array}{l}\text { Weighted } \\
\text { Frequency }\end{array}$ & $\begin{array}{l}\text { Decision } \\
\text { Round }\end{array}$ & Reason Excluded & $\mathrm{IoH}$ \\
\hline 773 & $\begin{array}{l}\text { Other person consult on behalf of } \\
\text { another... }\end{array}$ & V6519 & 3406 & $\mathrm{EC}$ & No.Consensus & 19 \\
\hline 813 & Other Streptococcus bacterial infection & 4109 & 3798 & $\mathrm{EC}$ & Too.Vague & 44 \\
\hline 827 & Other specified diffuse diseases of c. . & 7108 & 37630 & $\mathrm{EC}$ & Too.Vague & 38 \\
\hline 843 & Personal history of other injury & V1559 & 55619 & $\mathrm{EC}$ & Too.Vague & 27 \\
\hline 852 & Personal history of other hazards to ... & V1589 & 84508 & $\mathrm{EC}$ & Too.Vague & 27 \\
\hline 856 & Alcohol withdrawal delirium & 2910 & 28236 & $\mathrm{EC}$ & No.Consensus & 78 \\
\hline 861 & Other specified viral infection & 7989 & 2557 & $\mathrm{EC}$ & Too.Vague & 32 \\
\hline 867 & Special screening for malignant neopl. . . & V7644 & 124634 & $\mathrm{EC}$ & Too.Vague & 39 \\
\hline 870 & Special screening examination for ven. . . & V745 & 137618 & $\mathrm{EC}$ & Too.Vague & 39 \\
\hline 873 & Benign carcinoid tumor of unknown prim.. & 20960 & 9973 & $\mathrm{EC}$ & Too.Vague & 66 \\
\hline 881 & Surgical/oth proc not carried out bec... & V642 & 28045 & $\mathrm{EC}$ & No.Consensus & 14 \\
\hline 890 & Prophylactic vaccination/inoculation & V053 & 45360 & $\mathrm{EC}$ & No.Consensus & 46 \\
\hline 891 & Sleep related leg cramps & 32752 & 28430 & $\mathrm{EC}$ & No.Consensus & 39 \\
\hline 896 & Other specified delays in development & 3158 & 22020 & $\mathrm{EC}$ & Too.Vague & 55 \\
\hline 929 & Oth current conds class elsewhr, ante... & 64893 & 132471 & $\mathrm{EC}$ & Too.Vague & 36 \\
\hline 932 & Hemophilus influenzae [H. influenzae]. . . & 415 & 9554 & $\mathrm{EC}$ & Too.Vague & 48 \\
\hline 942 & Examination following radiotherapy & V671 & 3742 & $\mathrm{EC}$ & Too.Vague & 28 \\
\hline 950 & Glossopharyngeal neuralgia & 3521 & 7687 & $\mathrm{EC}$ & No.Consensus & 48 \\
\hline 952 & Angioneurotic edema & 9951 & 67242 & $\mathrm{EC}$ & No.Consensus & 57 \\
\hline 973 & Contact with or exposure to varicella & V0171 & 6501 & $\mathrm{EC}$ & No.Consensus & 29 \\
\hline 974 & Human papilloma virus & 794 & 11439 & $\mathrm{EC}$ & No.Consensus & 44 \\
\hline 977 & Abdominal or pelvic swelling, mass, o... & 78930 & 82946 & $\mathrm{EC}$ & Too.Vague & 62 \\
\hline 980 & Coxsackie virus & 792 & 6288 & $\mathrm{EC}$ & Too.Vague & 41 \\
\hline 989 & Special screening for unspecified con. & V829 & 24545 & $\mathrm{EC}$ & Too.Vague & 24 \\
\hline 999 & Other lymphedema & 4571 & 12447 & $\mathrm{EC}$ & No.Consensus & 41 \\
\hline 1016 & Polyphagia & 7836 & 42397 & $\mathrm{EC}$ & No.Consensus & 37 \\
\hline 1021 & Lack of adequate sleep & V694 & 13326 & $\mathrm{EC}$ & Too.Vague & 34 \\
\hline 1038 & Other specified contraceptive management & V258 & 80541 & $\mathrm{EC}$ & No.Consensus & 47 \\
\hline 1068 & $\begin{array}{l}\text { Other nongonococcal urethritis } \\
{[\mathrm{NGU}], \ldots}\end{array}$ & 9940 & 13006 & $\mathrm{EC}$ & No.Consensus & 52 \\
\hline 1072 & Open wound of upper arm, complicated & 88013 & 9986 & $\mathrm{EC}$ & Too.Vague & 50 \\
\hline 1082 & Other and unspecified diseases of the... & 5289 & 173005 & $\mathrm{EC}$ & No.Consensus & 34 \\
\hline 1084 & Acquired deformity of other specified. . & 7388 & 4342 & $\mathrm{EC}$ & Too.Vague & 28 \\
\hline 1089 & Benign neoplasm of tongue & 2101 & 37630 & $\mathrm{EC}$ & No.Consensus & 34 \\
\hline 1108 & Abnormal chest sounds & 7867 & 37630 & $\mathrm{EC}$ & Too.Vague & 45 \\
\hline 1154 & Leukorrhea, not specified as infective & 6235 & 159964 & $\mathrm{EC}$ & No.Consensus & 29 \\
\hline 1156 & Contusion of face, scalp, and neck ex. . . & 920 & 112907 & EC & No.Consensus & 34 \\
\hline 1174 & Benign neoplasm of other specified sites & 2298 & 16425 & $\mathrm{EC}$ & Too.Vague & 31 \\
\hline 1182 & Unspecified viral infection & 7999 & 521567 & $\mathrm{EC}$ & Too.Vague & 34 \\
\hline 1193 & Contusion of unspecified site & 9249 & 220991 & EC & Too.Vague & 29 \\
\hline 1221 & Foreign body in mouth & 9350 & 4371 & $\mathrm{EC}$ & No.Consensus & 41 \\
\hline 1239 & Other nonspecific abnormal findings & 7969 & 9388 & $\mathrm{EC}$ & Too.Vague & 28 \\
\hline 1263 & Flushing & 78262 & 16935 & $\mathrm{EC}$ & No.Consensus & 26 \\
\hline 1266 & Candidiasis of unspecified site & 1129 & 169614 & $\mathrm{EC}$ & No.Consensus & 32 \\
\hline 1275 & Special screening for other/unspec ca. . & V812 & 164577 & $\mathrm{EC}$ & Too.Vague & 44 \\
\hline 1278 & Unspecified vitamin B deficiency & 2669 & 47238 & $\mathrm{EC}$ & Too.Vague & 41 \\
\hline 1288 & Other counseling and advice for contr... & V2509 & 365741 & $\mathrm{EC}$ & No.Consensus & 47 \\
\hline 1302 & Contact with or exp to other communic. . & V0189 & 36617 & $\mathrm{EC}$ & Too.Vague & 35 \\
\hline
\end{tabular}




\begin{tabular}{|c|c|c|c|c|c|c|}
\hline Item & ICD-9 Description & $\begin{array}{l}\text { ICD-9 } \\
\text { Code }\end{array}$ & $\begin{array}{l}\text { Weighted } \\
\text { Frequency }\end{array}$ & $\begin{array}{l}\text { Decision } \\
\text { Round }\end{array}$ & Reason Excluded & $\mathrm{IoH}$ \\
\hline 1303 & Polyarticular juvenile rheumatoid art. . . & 71430 & 7136 & $\mathrm{EC}$ & No.Consensus & 65 \\
\hline 1319 & Disorders of soft tissue, unspecified & 72990 & 12647 & $\mathrm{EC}$ & Too.Vague & 35 \\
\hline 1322 & Swelling of limb & 72981 & 342354 & $\mathrm{EC}$ & Too.Vague & 49 \\
\hline 1328 & Mechan complic due to oth implnt/inte... & 99659 & 18558 & $\mathrm{EC}$ & Too.Vague & 47 \\
\hline 1341 & Infected postoperative seroma & 99851 & 3798 & $\mathrm{EC}$ & Too.Vague & 52 \\
\hline 1385 & Contusion of buttock & 92232 & 46913 & $\mathrm{EC}$ & No.Consensus & 32 \\
\hline 1391 & Genital herpes, unspecified & 5410 & 66679 & $\mathrm{EC}$ & Too.Vague & 46 \\
\hline 1406 & Polymyalgia rheumatica & 725 & 97420 & $\mathrm{EC}$ & No.Consensus & 60 \\
\hline 1407 & Special screening examination for mal. . & V751 & 17594 & $\mathrm{EC}$ & No.Consensus & 32 \\
\hline 1446 & $\begin{array}{l}\text { Other and unspec Escherichia coli } \\
\text { [E. coli] }\end{array}$ & 4149 & 3177 & $\mathrm{EC}$ & Too.Vague & 50 \\
\hline 1470 & Gilles de la Tourette`s disorder & 30723 & 15223 & $\mathrm{EC}$ & No.Consensus & 50 \\
\hline 1471 & Other chronic pain & 33829 & 2012835 & $\mathrm{EC}$ & Too.Vague & 59 \\
\hline 1473 & Streptococcus, Group B bacterial infe. . . & 4102 & 16950 & $\mathrm{EC}$ & Too.Vague & 50 \\
\hline 1491 & Tracheoesophageal fistula, esophageal. . . & 7503 & 5234 & $\mathrm{EC}$ & No.Consensus & 66 \\
\hline 1495 & Other healthy infant or child receivi. . & V201 & 22341 & $\mathrm{EC}$ & Too.Vague & 30 \\
\hline 1498 & Decreased libido & 79981 & 39003 & $\mathrm{EC}$ & No.Consensus & 40 \\
\hline 1504 & Urethritis, unspecified & 59780 & 82696 & $\mathrm{EC}$ & No.Consensus & 53 \\
\hline 1521 & Exposure to mold & V8731 & 17594 & $\mathrm{EC}$ & No.Consensus & 30 \\
\hline 1612 & Neonatal Candida infection & 7717 & 18061 & $\mathrm{EC}$ & Too.Vague & 47 \\
\hline 1615 & Personal history of other infectious ... & V1209 & 97978 & $\mathrm{EC}$ & Too.Vague & 39 \\
\hline 1619 & Prophylactic vaccin/inoculatn agnst m. . & V064 & 24878 & $\mathrm{EC}$ & No.Consensus & 45 \\
\hline 1625 & Sciatia & 7243 & 860164 & $\mathrm{EC}$ & No.Consensus & 42 \\
\hline 1631 & Other specified preoperative examination & V7283 & 253185 & $\mathrm{EC}$ & No.Consensus & 39 \\
\hline 1644 & $\begin{array}{l}\text { Open wound of hip and thigh with } \\
\text { tend... }\end{array}$ & 8902 & 1985 & $\mathrm{EC}$ & No.Consensus & 60 \\
\hline 1647 & Other disorders of soft tissue & 72999 & 4074 & $\mathrm{EC}$ & Too.Vague & 33 \\
\hline 1680 & Neoplasms of unspecified nature site ... & 2399 & 12719 & $\mathrm{EC}$ & Too.Vague & 50 \\
\hline 1682 & Contusion of unspecified part of lowe... & 9245 & 51593 & $\mathrm{EC}$ & No.Consensus & 22 \\
\hline 1699 & Contusion of thigh & 92400 & 10432 & $\mathrm{EC}$ & No.Consensus & 15 \\
\hline 1701 & Herpetic gingivostomatitis & 542 & 5987 & $\mathrm{EC}$ & No.Consensus & 40 \\
\hline 1706 & Late effects of other and unspec infe... & 1398 & 28430 & $\mathrm{EC}$ & Too.Vague & 42 \\
\hline 1729 & Other ventral hernia & 55329 & 4511 & $\mathrm{EC}$ & No.Consensus & 36 \\
\hline 1731 & Abnormality of gait & 7812 & 142207 & $\mathrm{EC}$ & No.Consensus & 40 \\
\hline 1736 & $\begin{array}{l}\text { Oth \& unspec spec } \\
\text { symptoms/syndromes, ... }\end{array}$ & 3079 & 7312 & $\mathrm{EC}$ & Too.Vague & 42 \\
\hline 1737 & Other symptoms involving head and neck & 78499 & 149981 & $\mathrm{EC}$ & Too.Vague & 42 \\
\hline 1744 & Anaphylactic shock due to peanuts & 99561 & 12776 & $\mathrm{EC}$ & No.Consensus & 91 \\
\hline 1746 & Premenstrual tension syndromes & 6254 & 118033 & $\mathrm{EC}$ & No.Consensus & 36 \\
\hline 1759 & Unspecified chlamydial infection & 7998 & 4328 & $\mathrm{EC}$ & Too.Vague & 54 \\
\hline 1762 & Special screeng exam for oth spec bac. . & V748 & 14753 & $\mathrm{EC}$ & Too.Vague & 36 \\
\hline 1773 & Other hyperalimentation & 2788 & 16399 & $\mathrm{EC}$ & No.Consensus & 42 \\
\hline 1774 & Shortness of breath & 78605 & 479608 & $\mathrm{EC}$ & No.Consensus & 85 \\
\hline 1776 & Hepatic coma & 5722 & 9554 & $\mathrm{EC}$ & No.Consensus & 72 \\
\hline 1777 & Other abnormal clinical findings & 7964 & 84634 & $\mathrm{EC}$ & Too.Vague & 30 \\
\hline 1782 & Other drug allergy & 99527 & 113349 & $\mathrm{EC}$ & Too.Vague & 45 \\
\hline 1790 & Contusion of finger & 9233 & 88972 & $\mathrm{EC}$ & No.Consensus & 20 \\
\hline 1791 & Personal history of unspecified disease & V139 & 14372 & $\mathrm{EC}$ & Too.Vague & 30 \\
\hline 1792 & Staphylococcus bacterial infection, u. . . & 4110 & 18347 & $\mathrm{EC}$ & Too.Vague & 59 \\
\hline
\end{tabular}




\begin{tabular}{|c|c|c|c|c|c|c|}
\hline Item & ICD-9 Description & $\begin{array}{l}\text { ICD-9 } \\
\text { Code }\end{array}$ & $\begin{array}{l}\text { Weighted } \\
\text { Frequency }\end{array}$ & $\begin{array}{l}\text { Decision } \\
\text { Round }\end{array}$ & Reason Excluded & $\mathrm{IoH}$ \\
\hline 1797 & Injury to unspecified site & 9599 & 92535 & $\mathrm{EC}$ & Too.Vague & 30 \\
\hline 1806 & Injury of face and neck & 95909 & 30920 & $\mathrm{EC}$ & Too.Vague & 54 \\
\hline 1813 & Other cardiorespiratory problems & V472 & 28430 & $\mathrm{EC}$ & Too.Vague & 63 \\
\hline 1825 & Other chest pain & 78659 & 321974 & $\mathrm{EC}$ & Too.Vague & 54 \\
\hline 1833 & Aftercare following surgery genito sys, nec & V5876 & 28430 & $\mathrm{EC}$ & No.Consensus & 40 \\
\hline 1834 & Oth/unspec superfic injury of hip/thi. . & 9168 & 12393 & $\mathrm{EC}$ & No.Consensus & 35 \\
\hline 1841 & $\begin{array}{l}\text { Spinal stenosis, lumbar region, w/neuro } \\
\text { claudication }\end{array}$ & 72402 & 103840 & $\mathrm{EC}$ & No.Consensus & 58 \\
\hline 1853 & Anorexia & 7830 & 92742 & $\mathrm{EC}$ & No.Consensus & 62 \\
\hline 1863 & Diabetes w/ophthalmic manifestations,. . . & 25050 & 136985 & $\mathrm{EC}$ & No.Consensus & 66 \\
\hline 1870 & Ot venereal dis due to Chlamydia trac... & 9954 & 7140 & $\mathrm{EC}$ & Too.Vague & 54 \\
\hline 1889 & Other specified noninflammatory disor... & 6248 & 31480 & $\mathrm{EC}$ & Too.Vague & 31 \\
\hline 1891 & Contusion of lower leg & 92410 & 34756 & $\mathrm{EC}$ & No.Consensus & 22 \\
\hline
\end{tabular}

Note: Please contact the corresponding author for more information regarding the data. 